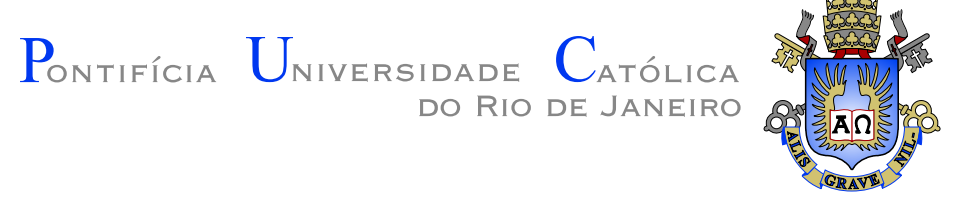

Rômulo César Costa de Sousa

\title{
Part-of-Speech Tagging para Português
}

Dissertação de Mestrado

Dissertação apresentada como requisito parcial para obtenção do grau de Mestre pelo Programa de Pós-graduação em Informática do Departamento de Informática da PUC-Rio.

Orientador: Prof. Hélio Côrtes Vieira Lopes 


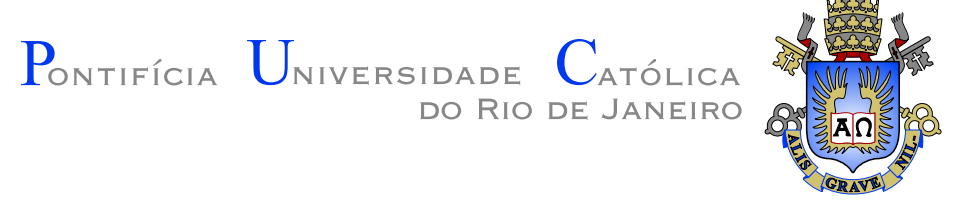

Rômulo César Costa de Sousa

Part-of-Speech Tagging para Português

Dissertação apresentada como requisito parcial para obtenção do grau de Mestre pelo Programa de Pós-graduação em Informática do Departamento de Informática da PUC-Rio. Aprovada pela Comissão Examinadora abaixo assinada.

Prof. Hélio Côrtes Vieira Lopes

Orientador

Departamento de Informática - PUC-Rio

Prof. Eduardo Sany Laber

Departamento de Informatica - PUC-Rio

Prof. Marco Antonio Casanova

Departamento de Informatica - PUC-Rio

Rio de Janeiro, 9 Agosto 2019 
Todos os direitos reservados. É proibida a reprodução total ou parcial do trabalho sem autorização da universidade, do autor e do orientador.

\section{Rômulo César Costa de Sousa}

Bacharel em Ciência da Computação (2016) na Universidade Federal do Ceará - Campus Quixadá (UFC)

Ficha Catalográfica

Rômulo César Costa de Sousa

Part-of-Speech Tagging para Português / Rômulo César Costa de Sousa; orientador: Hélio Côrtes Vieira Lopes. - Rio de janeiro: PUC-Rio, Departamento de Informática, 2019.

v., 86 f: il. color. ; $30 \mathrm{~cm}$

Dissertação (mestrado) - Pontifícia Universidade Católica do Rio de Janeiro, Departamento de Informática.

Inclui bibliografia

1. Processamento de Linguagem Natural. 2. Anotação Morfossintática. 3. Aprendizado de Máquina. 4. Aprendizagem Profunda. 5. Incorporação de Palavras.

I. Hélio Côrtes Vieira Lopes. II. Pontifícia Universidade Católica do Rio de Janeiro. Departamento de Informática. III. Título. 


\section{Agradecimentos}

Primeiramente gostaria de agradecer a Deus, por ter me concedido saúde, força e disposição.

Sou imensamente grato ao Prof. Hélio Lopes grande professor e orientador. Muito obrigado pela paciência, orientação, confiança e dedicação.

Gostaria de agradecer imensamente aos meus pais, Antonio César e Ocilene, as minhas irmãs Jessyca e Isabelly, que nunca negaram apoio durante minha trajetória acadêmica.

A todos os amigos da pós-graduação, especialmente André, Micaele, Dalai, Lauro, Vinicius e Luisa, meu muito obrigado. Vocês foram fundamentais para minha formação, por isso merecem o meu eterno agradecimento.

Agradeço a Welida, sem o seu apoio e companheirismo esse trabalho não seria possível. Obrigado por ser tão atenciosa.

Agradeço a todos os integrantes dos laboratórios DasLab e Ideias, por me proporcionar um ambiente criativo, amigável e colaborativo para os estudos e pesquisas.

O presente trabalho foi realizado com apoio da Coordenação de Aperfeiçoamento de Pessoal de Nível Superior - Brasil (CAPES) - Código de Financiamento 001. 


\section{Resumo}

Rômulo César Costa de Sousa; Hélio Côrtes Vieira Lopes. Partof-Speech Tagging para Português. Rio de Janeiro, 2019. 86p. Dissertação de Mestrado - Departamento de Informática, Pontifícia Universidade Católica do Rio de Janeiro.

Part-of-speech (POS) tagging é o processo de categorizar cada palavra de uma sentença com sua devida classe morfossintática (verbo, substantivo, adjetivo e etc). POS tagging é considerada uma atividade fundamental no processo de construção de aplicações de processamento de linguagem natural (PLN), muitas dessas aplicações, em algum ponto, demandam esse tipo de informação. Nesse trabalho, construímos um POS tagger para o Português Contemporâneo e o Português Histórico, baseado em uma arquitetura de rede neural recorrente. Tradicionalmente a construção dessas ferramentas requer muitas features específicas do domínio da linguagem e dados externos ao conjunto de treino, mas nosso POS tagger não usa esses requisitos. Treinamos uma rede Bidirectional Long short-term memory (BLSTM), que se beneficia das representações de word embeddings e character embeddings das palavras, para atividade de classificação morfossintática. Testamos nosso POS tagger em três corpora diferentes: a versão original do corpus MacMorpho, a versão revisada do corpus Mac-Morpho e no corpus Tycho Brahe. Nós obtemos um desempenho ligeiramente melhor que os sistemas estado da arte nos três corpora: $97.83 \%$ de acurácia para o Mac-Morpho original, 97.65\% de acurácia para o Mac-Morpho revisado e 97.35\% de acurácia para Tycho Brahe. Conseguimos, também, uma melhora nos três corpora para a medida de acurácia fora do vocabulário, uma acurácia especial calculada somente sobre as palavras desconhecidas do conjunto de treino. Realizamos ainda um estudo comparativo para verificar qual dentre os mais populares algoritmos de criação de word embedding (Word2Vec, FastText, Wang2Vec e Glove), é mais adequado para a atividade POS tagging em Português. O modelo de Wang2Vec mostrou um desempenho superior.

\section{Palavras-chave}

Processamento de Linguagem Natural; Anotação Morfossintática; Aprendizado de Máquina; Aprendizagem Profunda; Incorporação de Palavras. 


\section{Abstract}

Rômulo César Costa de Sousa; Hélio Côrtes Vieira Lopes (Advisor). Part-of-Speech Tagging for Portuguese. Rio de Janeiro, 2019. 86p. Dissertação de mestrado - Departamento de Informática, Pontifícia Universidade Católica do Rio de Janeiro.

Part-of-speech (POS) tagging is a process of labeling each word in a sentence with a morphosyntactic class (verb, noun, adjective and etc). POS tagging is a fundamental part of the linguistic pipeline, most natural language processing (NLP) applications demand, at some step, part-of-speech information. In this work, we constructed a POS tagger for Contemporary Portuguese and Historical Portuguese, using a recurrent neural network architecture. Traditionally the development of these tools requires many handcraft features and external data, our POS tagger does not use these elements. We trained a Bidirectional Long short-term memory (BLSTM) network that benefits from the word embeddings and character embeddings representations of the words, for morphosyntactic classification. We tested our POS tagger on three different corpora: the original version of the Mac-Morpho corpus, the revised version of the Mac-Morpho corpus, and the Tycho Brahe corpus. We produce state-of-the-art POS taggers for the three corpora: $97.83 \%$ accuracy on the original Mac-Morpho corpus, 97.65\% accuracy on the revised Mac-Morpho and $97.35 \%$ accuracy on the Tycho Brahe corpus. We also achieved an improvement in the three corpora in out-of-vocabulary accuracy, that is the accuracy on words not seen in training sentences. We also performed a comparative study to test which different types of word embeddings (Word2Vec, FastText, Wang2Vec, and Glove) is more suitable for Portuguese POS tagging. The Wang2Vec model showed higher performance.

\section{Keywords}

Natural Language Processing; Part-of-speech Tagging; Machine Learning; Deep Learning; Word Embedding. 


\section{Sumário}

1 Introdução 13

2 Redes neurais $\quad 16$

$\begin{array}{lll}2.1 & \text { Recurrent Neural Network (RNN) } & 17\end{array}$

\begin{tabular}{ll}
2.2 & Long short-term memory (LSTM) \\
\hline
\end{tabular}

2.3 Bidirectional Long short-term memory (BLSTM) 20

2.4 Dropout 20

3 Processamento de Linguagem Natural $\quad 22$

3.1 Processamento de Linguagem Natural e Redes Neurais 23

3.2 Representação vetorial de palavras 24

3.2.1 Word Embeddings 24

3.2.1.1 Word2vec 25

3.2.1.2 Glove 27

3.2.1.3 Wang2Vec 27

$\begin{array}{lll}3.2 .1 .4 & \text { FastText } & 27\end{array}$

3.2.2 Character Embeddings 28

4 Trabalhos Relacionados $\quad 29$

4.1 POS tagging para Português $\quad 29$

4.2 POS tagging para outros idiomas 31

5 Metodologia 33

5.1 Arquitetura de Rede Neural 33

6 Experimentos $\quad 36$

6.1 Corpora 36

6.1.1 Mac-Morpho v1 37

6.1.2 Mac-Morpho v2 40

6.1.3 Corpus Tycho Brahe 41

6.2 Métricas de avaliação $\quad 45$

6.3 Baselines 45

6.4 Ajuste de Hiperparâmetro 46

6.5 Análise de word embedding 49

6.5.1 Comparação com baselines $\quad 52$

6.5.2 Comparação com outros trabalhos $\quad 55$

6.6 Análise por etiquetas $\quad 57$

$\begin{array}{lll}6.7 & \text { Análise de erro } & 64\end{array}$

6.8 Análise de character embedding 64

7 Conclusões $\quad 67$

$\begin{array}{lll}7.1 & \text { Resumo do trabalho } & 67\end{array}$

$\begin{array}{lll}7.2 & \text { Contribuições } & 68\end{array}$

$\begin{array}{lll}7.3 & \text { Trabalhos futuros } & 68\end{array}$ 
$\begin{array}{lll}\text { A Descrição das etiquetas do Tycho Brahe corpus } & 77\end{array}$

B Avaliação modelo BLSTM-WE-CE no corpus Tycho Brahe. 


\section{Lista de figuras}

$\begin{array}{lll}\text { Figura 2.1 Uma rede neural simples } & 16\end{array}$

$\begin{array}{lll}\text { Figura 2.2 Uma rede neural simples com camada escondida } & 17\end{array}$

Figura 2.3 Uma rede neural recorrente e o desdobramento no tempo de computação

Figura 2.4 Célula LSTM. Adaptada de (56) 19

Figura 2.5 Estrutura de RNN unidirecional e bidirecional 20

Figura 2.6 a) Uma rede neural padrão. b) Um exemplo de uma rede produzida pela aplicação de dropout na rede à esquerda. (66) 21

Figura 3.1 Exemplo de operação entre vetores, adaptada de (26) 25

Figura 3.2 Modelo CBOW de (59) 26

Figura 3.3 Modelo Skip Gram de (59) 26

Figura 5.1 Modelo BLSTM-WE-CE. Arquitetura da rede neural para POS tagging. $\quad 35$

Figura 5.2 Arquitetura do character embeddings 35

Figura 6.1 Distribuição das etiquetas no Mac-Morpho v1 37

Figura 6.2 Distribuição das palavras com o maior número de diferentes etiquetas associadas. Corpus Mac-Morpho v1. 40

Figura 6.3 Nuvem de palavras Mac-Morpho v1 40

Figura 6.4 Distribuição das etiquetas no Mac-Morpho v2 41

Figura 6.5 Nuvem de palavras Mac-Morpho v2 42

Figura 6.6 Distribuição das etiquetas no Tycho Brahe 44

Figura 6.7 Nuvem de palavras Tycho Brahe 44

Figura 6.8 Arquitetura do baseline Perceptron-featuresmanuais

Figura 6.9 Arquitetura de baseline BLSTM-WE.

Figura 6.10 Treino do modelo BLSTM-WE-CE nas corpora MacMorpho v1, Mac-Morpho v2 e Tycho Brahe.

Figura 6.11 Boxplot dos resultados de 10 diferentes treinos com o modelo BLSTM-WE-CE nos corpora Mac-Morpho v1, MacMorpho v2 e Tycho Brahe.

Figura 6.12 Valor médio de $F_{1}$ dos 10 experimentos realizados com o modelo BLSTM-WE-CE no corpus Mac-Morpho v1.

Figura 6.13 Valor médio de $F_{1}$ dos 10 experimentos realizados com o modelo BLSTM-WE-CE no corpus Mac-Morpho v2.

Figura 6.14 Valor médio de $F_{1}$ dos 10 experimentos realizados com o modelo BLSTM-WE-CE no corpus TychoBrahe para as 15 etiquetas mais frequentes.

Figura 6.15 Histograma: quantidade de etiquetas em 5 grupo de avaliação de $F_{1}$

Figura 6.16 Gráfico de correlação do valor $F_{1}$ da etiqueta pelo logaritmo do número de exemplos. 


\section{Lista de tabelas}

$\begin{array}{lll}\text { Tabela } 1.1 & \text { Exemplo de POS tagging } & 13\end{array}$

Tabela 4.1 Trabalhos relacionados para POS tagging em Português 31

Tabela 4.2 Trabalhos relacionados para POS tagging em outros idiomas

Tabela 6.1 Partições dos Corpora 36

Tabela 6.2 Descrição do corpus Mac-Morpho v1 38

Tabela 6.3 Distribuição das etiquetas no corpus Mac-Morpho v1 39

Tabela 6.4 Descrição do corpus Mac-Morpho v2 42

Tabela 6.5 Distribuição das etiquetas no corpus Mac-Morpho v2 43

Tabela 6.6 Resultados dos experimentos do modelo Perceptronfeatures-manuais avaliado no conjunto de desenvolvimento do Mac-Morpho v1.

Tabela 6.7 Resultados dos experimentos do modelo BLSTM-WE avaliado no conjunto de desenvolvimento do Mac-Morpho v1.

Tabela 6.8 Resultados dos experimentos do modelo BLSTM-WE$\mathrm{CE}$ avaliado no conjunto de desenvolvimento do Mac-Morpho v1.

Tabela 6.9 Hiperparâmetros selecionados para as arquiteturas baseline e arquitetura principal.

Tabela 6.10 Desempenho do nosso modelo com diferentes word embeddings no conjunto validação.

Tabela 6.11 Desempenho dos nossos modelos baseline no conjunto de teste

Tabela 6.12 Tempo de treino aproximado nos três diferentes corpora

Tabela 6.13 Resultados no conjunto de teste para dez treinos diferentes com sementes aleatórias.

Tabela 6.14 Comparação com outros POS taggers em Português

Tabela 6.15 Valor médio de $F_{1}$, Precision e Recall dos experimentos realizados com o BLSTM-WE-CE no conjunto de teste

Tabela 6.16 Valor médio de $F_{1}$, Precision e Recall por etiqueta no conjunto de treino do corpus Mac-Morpho v1

Tabela 6.17 Valor médio de $F_{1}$, Precision e Recall por etiqueta no conjunto de treino do corpus Mac-Morpho v2

Tabela 6.18 Os tokens com mais erros e as etiquetas que eles possuem no conjunto de teste do Mac-Morpho v1.

Tabela 6.19 Os tokens com mais erros e as etiquetas que eles possuem no conjunto de teste do Mac-Morpho v2.

Tabela 6.20 Os tokens com mais erros e as etiquetas que eles possuem no conjunto de teste do Tycho Brahe.

Tabela 6.21 Cinco palavras fora do vocabulário e suas palavras mais similares de acordo com a representação de character embedding 66

Tabela A.1 Distribuição das etiquetas no corpus Tycho Brahe 
Tabela A.2 Descrição e exemplos das etiquetas do corpus Tycho Brahe 81

Tabela B.1 Valor médio de $F_{1}$, Precision e Recall por etiqueta no conjunto de treino do corpus Tycho Brahe 
Tudo o que temos de decidir é o que fazer com o tempo que nos é dado

Gandalf, Senhor dos Anéis: A Sociedade do Anel 


\section{Introdução}

Entre o nascimento da Internet e 2003, o ano de nascimento das redes sociais (MySpace, LinkedIn e Facebook), foram produzidos apenas algumas dúzias de exabyte de informações em toda a Web. Atualmente, esse mesmo volume de informação é criado semanalmente. O advento das redes sociais ofereceu as pessoas uma nova forma de compartilhamento de conteúdo que permitem a criação e compartilhamento de ideias, opiniões e informações, tudo isso de maneira eficiente em relação a custo e tempo. Essa enorme quantidade de dados, entretanto, é produzida principalmente de forma não estruturada (pois é especificamente feita para o consumo humano) e, portanto, não são facilmente processáveis por máquinas. Processar automaticamente esse tipo de informação envolve uma profunda compreensão da linguagem natural por parte de sistemas de computação (50).

Processamento de linguagem natural (PLN) é a área multidisciplinar que explora como computadores podem usar, entender e manipular dados provenientes da linguagem natural, seja ela escrita ou falada (2). Dentre as principais aplicações de PLN estão incluídas análise de sentimentos, tradução automática, extração e recuperação de informações, sistemas de perguntas e respostas, reconhecimento de fala, verificação ortográfica, entre outras. $\mathrm{Na}$ construção de tais aplicações são empregadas ferramentas intermediárias que ajudam na manipulação e estruturação dos dados de linguagem natural, como por exemplo, Part-of-speech (POS) tagging, reconhecimento de entidade nomeada, reconhecimento de relações e análise sintática de dependência.

Em especial, Part-of-speech (POS) tagging é o processo de categorizar cada palavra de uma sentença com sua devida classe morfossintática. Na Tabela 1.1, temos um exemplo dessa classificação. A palavra "O" é um artigo, "cavalo" é um substantivo, "de" uma adposição, "Napoleão" outro substantivo, "é" verbo e "branco" um adjetivo.

Tabela 1.1: Exemplo de POS tagging

\begin{tabular}{l|l|l|l|l|l|l}
\hline Palavras & O & cavalo & de & Napoleão & é & branco \\
\hline Classes & ART & SUBS & ADP & SUBS & V & ADJ \\
\hline
\end{tabular}


POS tagging é uma tarefa não trivial devido ao fato de que algumas palavras podem ter mais de uma classe sintática dependendo do contexto em que elas são usadas. POS tagging é considerada uma atividade fundamental no processo construção de aplicações de PLN (12). Muitas aplicações, em algum ponto, demandam de informações de POS tagging (13). Por exemplo, é possível usar informações de POS tagging em sistemas de análise de sentimentos (14), tradução automática (15) e respostas automáticas (16).

Pesquisadores de PLN vêm fazendo um ótimo trabalho e definiram o estado da arte em acurácia de POS tagging para mais de 97\%. Porém, ainda assim, há espaço para melhorias, já que qualquer pequeno ganho em acurácia resulta em impactos em outras tarefas de PLN. Recentemente, técnicas de redes neurais se tornaram muito usadas para a resolução de problemas de PLN (51), bem como representações de word embeddings (25).

Em 2015, Wang et al(52) usaram uma rede neural recorrente (Bidirectional Long Short-Term Memory) combinado a word embeddings para resolver a tarefa de POS tagging para o inglês. A arquitetura foi testada no corpus Penn Treebank WSJ, que atingiu 97.40\% de acurácia. Em 2018, Bohnet et al. (1) definiram a pontuação estado da arte para o corpus Penn Treebank WSJ em inglês com 97.96\% de acurácia. Dos Santos e Zadrozny (44), construíram um modelo de rede neural para a resolução de POS tagging em Português. Eles aplicaram uma camada convolucional para extrair automaticamente features das palavras. Eles combinaram essa representação com a representação de word embeddings. O modelo foi avaliado em três corpora em Português: Mac-Morpho-v1, Mac-Morpho-v2 no corpus Tycho Brahe.

Este trabalho propõe construir um POS Tagger para a Língua Portuguesa usando uma arquitetura de redes neurais recorrentes que já se estabeleceram como estado da arte para POS Taggers em outros idiomas. Mais precisamente, a arquitetura proposta tem inspiração nos trabalhos de Ma e Hovy de 2016 (25) e Marulli et al. de 2018 (46).

Treinar POS taggers com desempenho estado da arte tradicionalmente requeira muitas features construídas de forma manual e dados externos ao corpus de treino. A arquitetura proposta nesse trabalho combina uma rede BLSTM com representações de word embeddings pré-treinados e representações de character embeddings para realizar a tarefa de POS tagging, sem usar quaisquer features manuais ou dados externos.

O modelo foi testado no Português Contemporâneo e no Português Histórico, usando três corpora: a versão original do Mac-Morpho corpus (v1) (47), a versão revisada do Mac-Morpho (v2) (48) e o corpus Tycho Brahe (49). Em todas os três corpora apresentamos um desempenho em acurácia e 
acurácia FV ligeiramente superior ao estado da arte reportado por Fonseca et al. (45) e Santos e Zadrozny (44). Obtivemos para o corpus Mac-Morpho v1, $97.83 \%$ de acurácia em média e $95.48 \%$ de acurácia FV em média. Para o corpus Mac-Morpho v2, 97.65\% de acurácia em média e $95.23 \%$ de acurácia FV em média. E para o corpus Tycho Brahe obtivemos, 97.35\% de acurácia em média e $87.40 \%$ de acurácia FV em média.

Podemos destacar como as principais contribuições desta dissertação os seguintes pontos:

- Demonstração empírica de que uso de redes BLSTM combinado a representações vetoriais de word embedding e character embedding são bem adequados e produzem bons resultado para o problema de POS tagging em Português.

- A criação de POS taggers para a Língua Portuguesa que superam ligeiramente o estado da arte em termos de acurácia e acurácia fora do vocabulário (FV), em três corpora diferentes.

- O estudo comparativo para verificar qual dentre os mais populares algoritmos de criação word embedding (Word2Vec, FastText, Wang2Vec e Glove), é mais adequado para a atividade POS tagging em Português. O modelo Wang2Vec mostrou um desempenho ligeiramente superior.

Este documento está organizado conforme descrito a seguir: nos Capítulos 2 e 3 temos a revisão bibliográfica, descrevendo conceitos sobre redes neurais e processamento de linguagem natural. No Capítulo 4 trazemos os trabalhos relacionados, dividido em sistemas de POS tagging para a Língua Portuguesa e sistemas de POS tagging para outras línguas. No Capítulo 5 apresentamos a metodologia do trabalho e descrição do modelo de rede neural. No Capítulo 6 apresentamos os corpora usados, as métricas de avaliação, descrevemos os experimentos e comparamos os resultados com o estado da arte. Por fim, no Capítulo 7 apresentamos as conclusões e trabalhos futuros. 


\section{2}

\section{Redes neurais}

Rede neural (RN) artificial tem sua inspiração no processo de aprendizado humano (20). Uma RN é composta de nós (neurônios) interligados, que recebem e fornecem informações. A mais simples versão de uma RN é composta de 2 camadas: camada de entrada e camada de saída, como ilustrado na Figura 2.1. Cada nó na camada de saída executa cálculos de pesos nos valores que recebem dos nós de entrada e então produzem valores de saída usando funções de transformação nos valores obtidos. Os pesos são corrigidos de acordo com erros ou perdas que a rede exibe nos nós de saída.

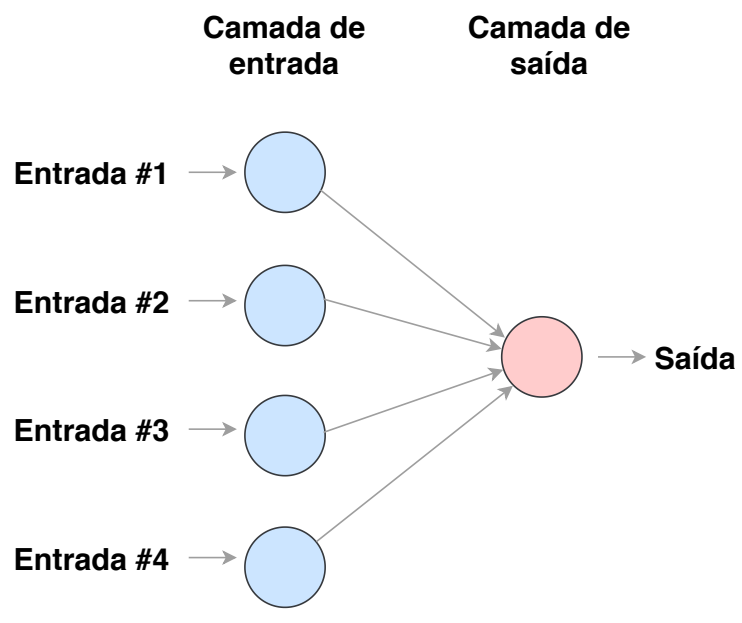

Figura 2.1: Uma rede neural simples

A camada da RN que fica entre a camada de entrada e a camada de saída é conhecida como camada escondida. Com a adição de camadas escondidas é possível separar os dados de uma forma não linear. O número de camadas escondidas e o número de nós em cada camada escondida são especificadas previamente e dependem da arquitetura aplicada. Na Figura 2.2 temos a ilustração de uma RN com uma camada de escondida. Por convenção, Deep Learning é a aplicação de redes neurais de múltiplas camadas para realizar tarefas de aprendizado, incluindo regressão, classificação, clusterização e outras (18). 


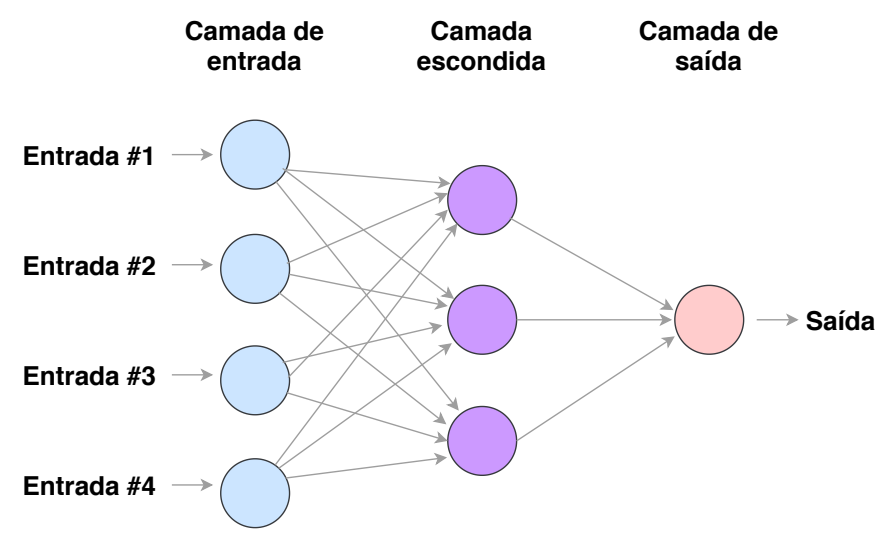

Figura 2.2: Uma rede neural simples com camada escondida

O conceito de RN foi introduzido no século XX (17) e, desde então, avanços na área vêm acontecendo. As técnicas de deep learning têm atraído o interesse de pesquisadores por sua capacidade de superar a desvantagens de algoritmos tradicionais que dependem de features projetadas manualmente. Abordagens de deep learning também se mostraram adequadas para análise em big data, aplicações em visão computacional, reconhecimento de padrões, processamento de linguagem natural e sistemas de recomendação (19).

\section{1}

\section{Recurrent Neural Network (RNN)}

Recurrent Neural Network (RNN), é uma família de redes neurais que processam dados sequenciais (23). As RNs tradicionais não consideram a sequência dos dados, porém em muitas aplicações isso se faz necessário. Se, por exemplo, queremos predizer a próxima palavra de uma sentença, é bem conveniente que conheçamos quais palavras ocorreram antes. RNNs comportam uma "memória" para armazenar informação do que já foi computado no passado. Na teoria, RNNs podem fazer o uso de informações de sequências arbitrariamente longas, mas na prática são limitadas apenas a informações de alguns passos anteriores.

RNNs contam com unidades de entrada $\left\{x_{0}, x_{1}, \ldots, x_{t}, x_{t+1}, \ldots\right\}$, unidades de saída $\left\{y_{0}, y_{1}, \ldots, y_{t}, y_{t+1}, \ldots\right\}$ e unidades escondidas $\left\{s_{0}, s_{1}, \ldots, s_{t}, s_{t+1}, \ldots\right\}$. Como ilustrado na Figura 2.3, no passo $t$, a RNN usa a amostra atual $x_{t}$ e a unidade escondida passada $s_{t-1}$ como entrada para obter o atual estado de $s_{t}$, com $s_{t}=f\left(x_{t}, s_{t-1}\right)$ onde $f$ denota a função de enconder (geralmente uma função não-linear, como tanh ou ReLU). Portanto, a RNN captura a dependência entre a amostra atual $x_{t}$ e a amostra anterior $x_{t-1}$ integrando a 
representação da unidade escondida $s_{t-1}(55)$.
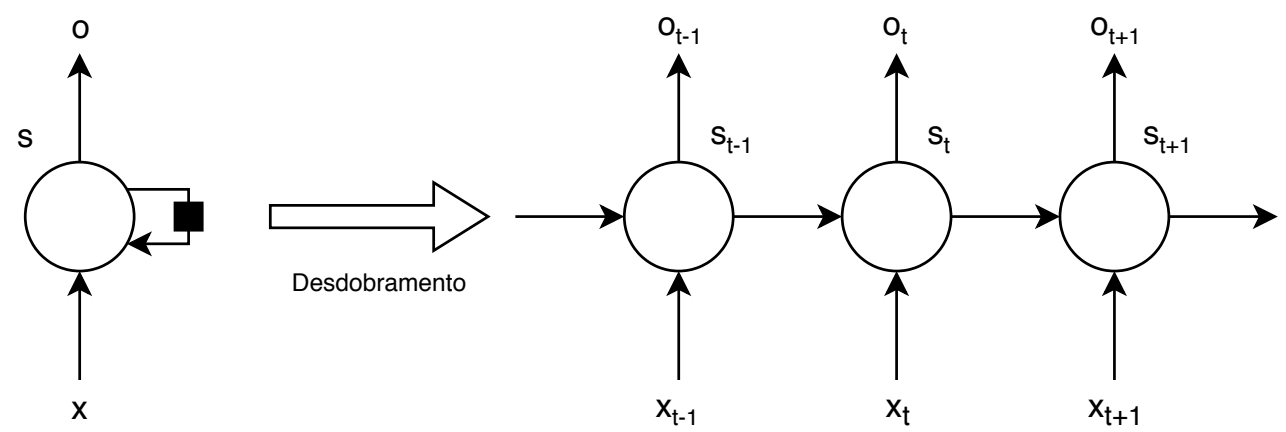

Figura 2.3: Uma rede neural recorrente e o desdobramento no tempo de computação

Durante o processo de treinamento das RNNs, uma função é usada para calcular o erro gerado pela a rede. Então o erro calculado é usado para estimar um gradiente que é usado para atualizar os pesos da rede, para que menos erros sejam cometidos na próxima iteração. Este gradiente é propagado por toda a rede, da camada de saída para a camada de entrada. Porém, na prática, realizar esse processo através de muitas camadas da rede pode fazer com que o valor de gradiente da função de erro se aproxime de zero, tornando toda a rede difícil de treinar. Esse problema é conhecido como gradient vanishing problem. RNN são redes poderosas, porém treiná-las tem se provado problemático por conta do problema do gradiente (24).

\section{2}

\section{Long short-term memory (LSTM)}

Long short-term memory (LSTM), proposta por Hochreiter e Schmidhuber em 1997 (22), é uma variante de RNN projetado para lidar com o gradient vanishing problem. LSTMs também tornam possível que RNNs lembrem de suas entradas por um maior período de tempo. Isso se deve ao fato que LSTMs contêm uma memória interna especial, que é parecida com a memória de um computador onde é possível ler, gravar e excluir e informações de computações anteriores.

Essa memória também pode ser vista como uma unidade composta por três portas multiplicativas, que controlam o fluxo da informação na rede, em que se pode esquecer informações (excluir) ou passar adiante para a próxima iteração. A Figura 2.4 descreve a estrutura básica de uma unidade de LSTM.

As três portas (input gates, forget gates e output gates) são combinadas para calcular o hidden state usando as seguintes equações (26): 


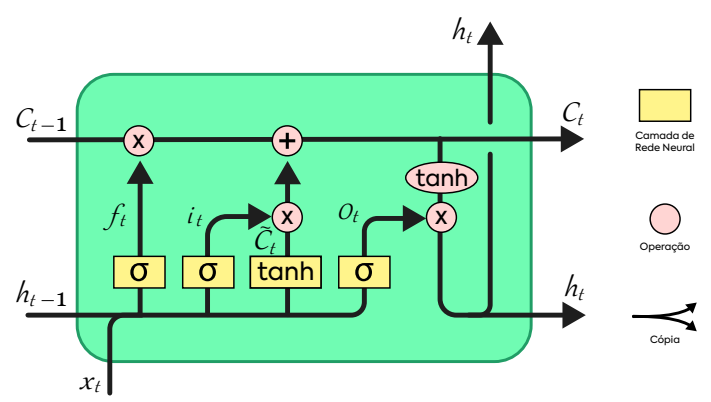

Figura 2.4: Célula LSTM. Adaptada de (56)

$$
\begin{gathered}
f_{t}=\sigma\left(W_{f} \cdot\left[h_{t-1}, x_{t}\right]+b_{f}\right) \\
i_{t}=\sigma\left(W_{i} \cdot\left[h_{t-1}, x_{t}\right]+b_{i}\right) \\
o_{t}=\sigma\left(W_{o} \cdot\left[h_{t-1}, x_{t}\right]+b_{o}\right) \\
c_{t}=f_{t} \odot c_{t-1}+i_{t} \odot \tanh \left(W_{c} \cdot\left[h_{t-1}, x_{t}\right]+b_{c}\right)
\end{gathered}
$$

O primeiro passo realizado dentro célula de LSTM é decidir quais informações serão descartadas da célula. Esta decisão é feita por uma camada sigmóide chamada de "forget gate". O calculo é feito na Equação 2-1, levando em conta o hidden state anterior $h_{t-1}$ e valor $x_{t}$ corrente. Uma saída de valor 1 representa "definitivamente mantenha esse valor" e uma saída de valor 0 representa "definitivamente esqueça esse valor".

O próximo passo é decidir quais novas informações serão armazenadas na célula. Primeiro, uma camada sigmóide chamada "input gate" decide quais valores serão atualizados, o calculo do "input gate" é feito na Equação 2-2. Depois, o valor $c_{t}$ corrente é atualizado usando o valor de $c_{t-1}$ anterior e o valor do forget gate, descrito na equação 2-4.

Finalmente, é calculado quais valores serão passados adiante na célula. Primeiro, é calculado o valor para "output gate" definido na equação 2-3. Após isso, o valor do hidden state atual $h_{t}$ é dado pela aplicação de uma função tanh no valor corrente de $c_{t}$ multiplicado por o output gate, como descrito na Equação 2-5.

Redes LSTM são aplicadas em grande uma variedade de atividades. Por exemplo, para reconhecimento facial em imagem (40), para analisar expressões faciais de indivíduos (53), para transcrever em linguagem natural cenas de vídeos (39), e até mesmo para gerar poemas de forma automática (38). 


\section{3}

\section{Bidirectional Long short-term memory (BLSTM)}

Em muitas tarefas com estruturas sequenciais, fazer o uso de informações passadas (à esquerda) e futuras (à direita) da sequência se torna uma vantagem. Porém, a arquitetura LSTM padrão conhece apenas informações de computações prévias (passado), e não conhece nada do futuro da sequência. Então, LSTM bidirecional (BLSTM) se apresenta como uma solução elegante para esse problema. A ideia é computar a sequência de frente para trás e de trás para frente, para capturar informações passadas e futuras (25). Tipicamente, BLSTM são mais poderosas que redes LSTM (41).

Inventada em 1997 por Schuster e Paliwal (54), as BLSTM aumentam a quantidade de informação de entrada disponível para a rede. Os neurônios são divididos em duas direções forward states e backward states. Na Figura 2.5 temos uma ilustração do processamento de sequência com uma RNN unidirecional e uma RNN bidirecional.
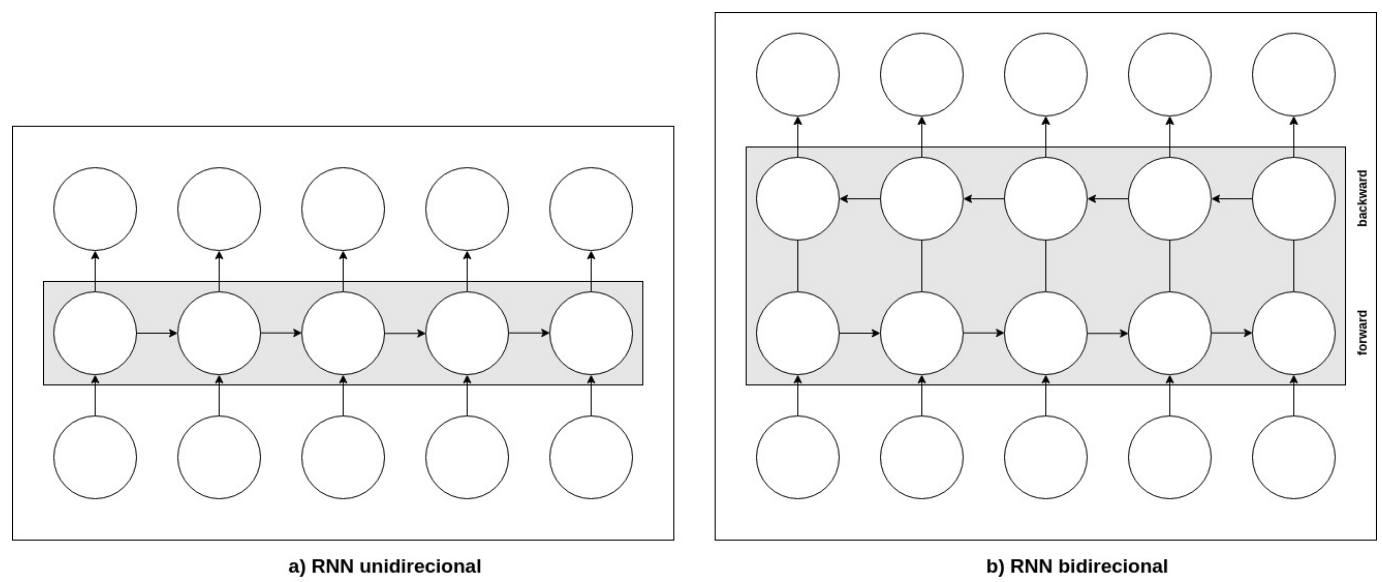

Figura 2.5: Estrutura de RNN unidirecional e bidirecional

\section{4}

\section{Dropout}

Redes neurais com um grande número de parâmetros são técnicas de aprendizado de máquina muito poderosas. No entanto, o overfitting é considerado um problema sério em redes neurais. O dropout é uma técnica que se propõe mitigar esse problema. A ideia é descartar aleatoriamente nós (juntamente com suas conexões) da rede durante o treinamento. Isso evita que os nós se adaptem demasiadamente aos dados de treino (66).

$\mathrm{Na}$ Figura 2.6 temos a ilustração da aplicação de dropout. Os nós são retirados de forma aleatória. Nos casos mais simples da aplicação de dropout, cada nó é retirado segundo uma probabilidade $p$ independente dos outros 
nós. Essa probabilidade $p$ é geralmente determinada usando o conjunto de validação. Segundo (66) 0.5 é um bom palpite para a escolha de $p$, pois é próximo da escolha ótima de uma grande variedade de redes neurais e tarefas.

O dropout é implementado como uma camada de rede neural. Ele pode ser usado combinado a vários tipos de camadas, como camadas densas totalmente conectadas, camadas convolucionais e camadas recorrentes, como LSTM e BLSTM. O dropout pode ser implementado em algumas camadas da rede ou até mesmo em todas as camadas escondidas. Geralmente não se usa dropout na camada de saída (60).

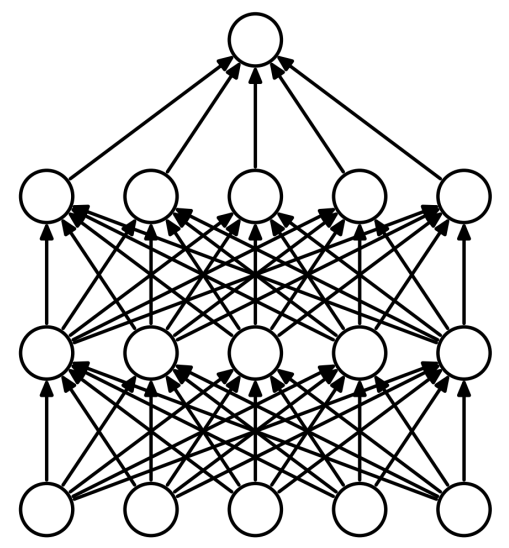

a) Rede Neural Padrão

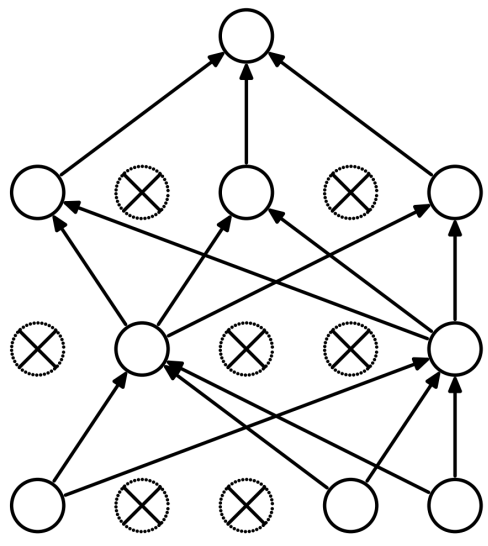

b) Depois de aplicar dropout

Figura 2.6: a) Uma rede neural padrão. b) Um exemplo de uma rede produzida pela aplicação de dropout na rede à esquerda. (66) 


\section{3}

\section{Processamento de Linguagem Natural}

Processamento de linguagem natural (PLN) é uma área de pesquisa que explora como computadores podem usar, entender e manipular a linguagem natural, seja ela escrita ou falada (2). PLN é uma área multidisciplinar que envolve campos de estudo como linguística, computação e estatística (4). Coppin e Ben (3) afirma que o objetivo final da área de pesquisa em PLN é construir sistemas com conhecimento suficiente para falar com humanos sobre qualquer assunto.

É cada vez mais importante que existam sistemas que possam compreender a linguagem natural de forma automática (3), pois o volume de dados que carregam linguagem natural está alcançando proporções de difícil gerenciamento. Por exemplo, por minuto na internet, mais de 16 milhões de mensagens de texto instantâneas são enviadas, mais de 156 milhões de e-mails são enviados (espera-se que até o fim de 2019 mais 9 bilhões de e-mails já tenham sido enviados) e são realizadas 154.200 chamadas no Skype (7).

A linguagem humana é complexa e diversa, tornando o processamento automático de linguagem uma tarefa não trivial. A ambiguidade da linguagem é um dos principais desafios em PLN.

Atualmente, computadores já são capazes de realizar uma grande variedade de atividades que envolvem linguagem natural, como por exemplo: reconhecimento de linguagem falada, sistemas de conversação automáticos, análise léxica, tradução automática, recuperação de informação, sistemas de perguntas e respostas, analise de sentimentos, geração de linguagem natural e sumarização de linguagem.

Para realizar tarefas tão complexas, as aplicações de PLN geralmente usam no processo de construção outros problemas mais fundamentais de PLN, como por exemplo: classificação gramatical de palavras (POS tagging), análise de sentenças e suas estruturas gramaticais, identificação de correlação de menções, reconhecimento de entidades nomeadas, entre outras.

As primeiras técnicas usadas em aplicações de PLN eram baseadas em regras. Cientistas escreviam de forma manual as regras de linguagem. Esse processo se provou uma tarefa difícil devido a ambiguidade presente na linguagem. Com o passar dos anos, PLN foi se transformando, e passaram 
a usar com sucesso métodos estatísticos e probabilísticos, juntamente com algoritmos de aprendizado de máquina (6).

Ao longo do tempo, em PLN, várias abordagens de aprendizado de máquina vêm sendo usadas como por exemplo: naive bayes (8), hidden markov models (9), support vector machines (10). Entretanto, durante os últimos anos, vem acontecendo uma nova transformação no campo de PLN, e muitos problemas que antes eram encarados com abordagem de aprendizado de máquina tradicional, agora apresentam bons resultados com aplicação de redes neurais (11).

\section{1}

\section{Processamento de Linguagem Natural e Redes Neurais}

Arquiteturas e algoritmos de redes neurais proporcionaram um grande avanço em áreas do conhecimento, como visão computacional e reconhecimento de padrões. Seguindo essa tendência, recentemente, pesquisadores de PLN estão voltando a atenção para o uso de técnicas de redes neurais. Nos últimos anos, redes neurais combinadas a representações vetoriais de palavras estão produzindo ótimos resultados em muitas atividades de PLN (26).

Em algoritmos de aprendizado de máquina tradicionais, as features são projetadas de forma manual, e engenharia de features, em muitos casos, é um gargalo pois requer conhecimentos bem específico de domínio. Deng e Liu (57) afirmam que engenharia de features era o maior obstáculo em PLN antes do advento de deep learning.

As técnicas atuais de deep learning contornam o problema de engenharia de features explorando poderosas redes neurais de múltiplas camadas que dispensam a necessidade da criação de features manuais. Ao contrário de algoritmos de aprendizagem de máquina mais tradicionais, algoritmos de deep learning são capazes de aprender representações a partir de dados usando uma hierarquia de múltiplas camadas para a geração automática de features (57).

Essa tendência surgiu, em parte, em 2011 com o trabalho pioneiro de Collobert el al. (42). Os autores aplicaram uma rede neural simples em vários problemas de PLN obtendo resultados que superavam o atual estado da arte em POS tagging, reconhecimento de entidade nomeada e anotação de papéis semânticos. Desde então redes neurais mais elaboradas vêm sendo empregas em inúmeros problemas de PLN. 


\section{2 \\ Representação vetorial de palavras}

Representação vetorial são basicamente uma forma de representar palavras que liga a compreensão humana da linguagem à de uma máquina. Essas representações são atualmente essenciais para resolver a maioria dos problemas da PLN (58).

\subsection{1}

\section{Word Embeddings}

Word Embeddings propõe representar cada palavra como um vetor de números reais em um espaço $D$-dimensional. Isso permite que palavras com significados similares tenham representações similares. A representação de Word embeddings captura informações semânticas e sintáticas das palavras (28).

Essa técnica de representação surge para combater a "maldição da dimensionalidade" (curse of dimensionality) (29), já que é possível representar as palavras de um vocabulário de tamanho $V$ com vetores $D$-dimensionais, em que $D$ pode ser muito menor que $V(D<<V)$.

É possível medir similaridade entre vetores de palavras usando funções de distância, como distância de cosseno, por exemplo. Também é possível realizar operações de soma e subtração entre pares de vetores e obter resultados com um significado semântico. Podemos, por exemplo subtrair os vetores vec("rei") e vec("homem") e somar com o vetor vec("mulher") e obter um resultado próximo do vetor vec("rainha"). Na Figura 3.1, temos a ilustração da operação de exemplo realizada na Equação 3-1.

$$
\operatorname{vec}\left(" r e i^{\prime \prime}\right)-\operatorname{vec}(\text { "homem") }+\operatorname{vec}(" m u l h e r ") \approx \operatorname{vec}(\text { (rainha"). }
$$

Word embeddings são bastante utilizados como a primeira camada de redes neurais. Normalmente esses vetores são pré-treinados em grande corpus não-supervisionados. (26). Existem uma boa variedade de algoritmos para gerar word embeddings: Word2vec (32), FastText (33), Wang2vec (34) e Glove (31) são alguns deles.

As representações de palavras por meio de word embeddings são bastante utilizadas na construção de sistemas de PLN (27) essa técnica vem sendo aplicada com bastante sucesso (25) na área.

Por exemplo, Ma e Hovy (25) propuseram uma arquitetura de rede neural que utiliza informações de word embedding para resolver de forma eficiente $P O S$ tagging e reconhecimento de entidades nomeadas. Dickinsonand e $\mathrm{Hu}$ (35) 
usaram word embedding junto com algoritmos de aprendizagem de máquina para identificar sentimentos em tweets.
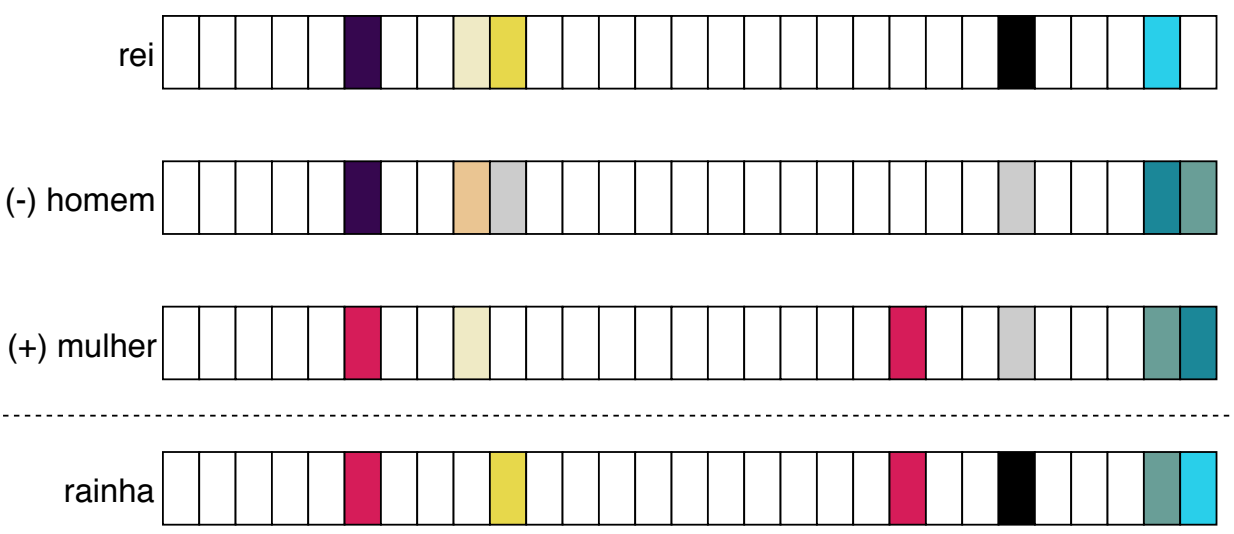

Figura 3.1: Exemplo de operação entre vetores, adaptada de (26)

\subsubsection{1}

\section{Word2vec}

Em 2013, Mikolov et al. (32) propuseram o Word2Vec, um modelo para construção de word embedding. Word2vec tem dois modelos de treinamento diferentes: (i) Continuous Bag-of-Words (CBOW) e (ii) skip-gram.

O modelo CBOW calcula a probabilidade condicional de uma palavra alvo específica, dadas as palavras de contexto que a rodeiam, através de uma janela de contexto de tamanho $C$. O modelo skip-gram faz exatamente o oposto do modelo CBOW, prevendo as palavras do contexto dado à palavra alvo específica.

O modelo CBOW é formado de uma simples rede neural fully connected com uma camada escondida, como exemplificado na Figura 3.2. A camada de entrada, que recebe os vetores das palavras do contexto, tem $V$ (tamanho do vocabulário) neurônios enquanto a camada oculta tem $N$ neurônios. A camada de saída é uma função softmax de todas as palavras no vocabulário. As camadas são conectas pelas matrizes de pesos $W^{V x N}$ e $W^{\prime N x V}$ (26) (60).

O modelo skip-gram usa a palavra alvo (cujo a representação queremos gerar) para prever as palavras do contexto e no processo, produz a representação vetorial da palavra. Na Figura 3.3 temos a representação do modelo skip-gram que também usa uma rede neural fully connected com três camadas.

Ambos modelos têm suas vantagens e desvantagens. De acordo com Mikolov (32), o modelo skip-gram funciona bem com pequenas quantidades 
de dados e representa bem as palavras raras. Entretanto, o modelos CBOW é mais rápido e gera melhores representações para palavras mais frequentes (60).

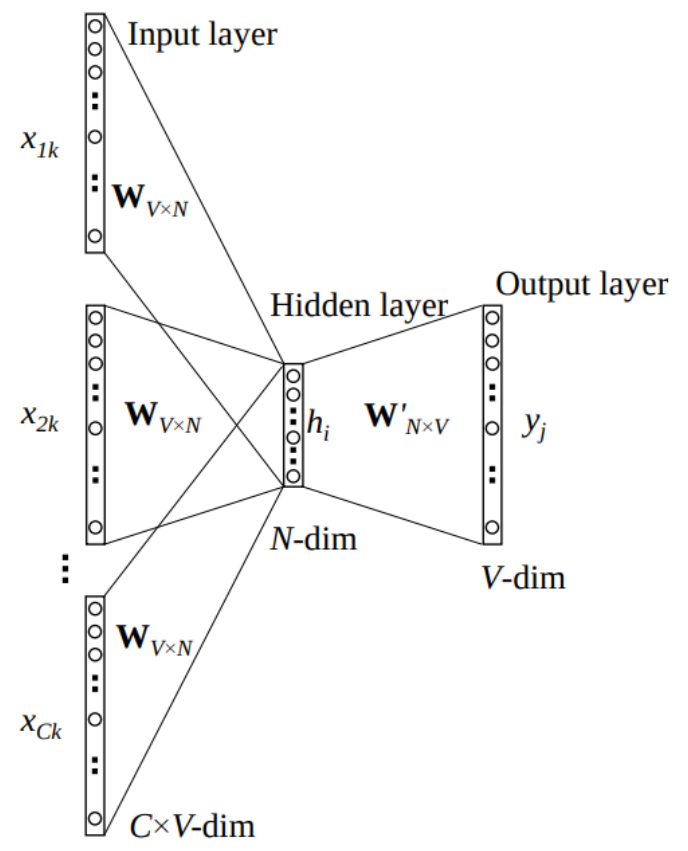

Figura 3.2: Modelo CBOW de (59)

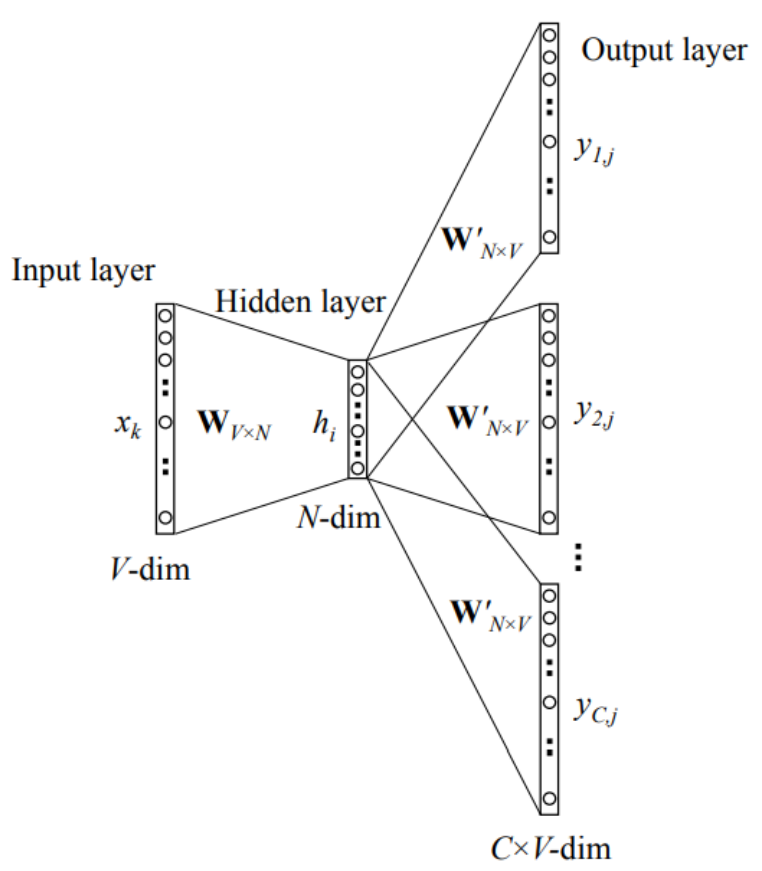

Figura 3.3: Modelo Skip Gram de (59) 


\subsubsection{2}

\section{Glove}

O método de geração de word embeddings Global Vectors (GloVe) foi proposto em 2014 por Pennington et al. em (31). Os autores observam que métodos baseados em janelas de contexto (CBOW e skip-gram) sofrem com a desvantagem de não aprender com estatísticas globais do corpus.

Para gera a representação de palavras usando estatísticas globais, o método Glove usa uma matriz de co-ocorrência $M$ que é construída usando as palavras do contexto. Cada elemento $M_{i j}$ na matriz representa a probabilidade da palavra $i$ estar perto da palavra $j$. Na matriz M, as linhas (ou vetores) são geradas aleatoriamente e treinado por obedecer a equação $P\left(w_{i}, w_{j}\right)=$ $\log \left(M_{i} j\right)=w_{i} w_{j}+b_{i}+b_{j}$ em que $w_{i}$ e $w_{j}$ são vetores e $b_{i}$ e $b_{j}$ são os biases (27).

\subsubsection{3}

\section{Wang2Vec}

O modelo Wang2Vec foi proposto por Wang Ling et al. (34) em 2015. Wang2Vec é uma modificação do Word2Vec feita a fim de considerar a ordem das palavras, o que não é considerado na arquitetura original. As modificações geram representações vetoriais que carregam um maior valor sintático. Foram feitas duas pequenas modificações. No modelo CBOW, a entrada passa a ser a concatenação de todas as palavras do contexto na exata ordem que elas aparecem. No modelo skip-gram, é usado um conjunto diferente de parâmetros para prever cada palavra de contexto, dependendo de sua posição em relação à palavra alvo (27).

\subsubsection{4}

\section{Fast Text}

O modelo FastText foi proposto em 2017 por Bojanowski et al. em (33). Os autores propuseram uma nova abordagem baseada no modelo skipgram de (32), onde cada palavra é representada como um conjunto de $n$-grams de caracteres. A representação vetorial de uma palavra específica é formada pela soma das representações dos n-grams que compõem a palavra. Com isso o modelo gera vetores de palavras que tem informações morfológica mais ricas. 


\subsection{2}

\section{Character Embeddings}

Character embeddings assim como word embeddings, é uma forma de representar palavras em um espaço vetorial de baixa dimensionalidade. Enquanto word embeddings são capazes de capturar informações sintáticas e semânticas das palavras, character embeddings capturam informações morfológicas e informações estruturais das palavras.

Informações morfológicas de palavras podem ser úteis em tarefas de classificação como POS tagging. Prefixos, sufixos, letras maiúsculas, entre outros, podem ajudar a distinguir a classe gramatical de palavras. Por exemplo, o sufixo "mente" da palavra "calmamente" pode indicar a presença de um advérbio. A letra maiúscula em "Maria" indica a presença de um substantivo.

Aplicações que trabalham com idiomas que tem um vocabulário grande, frequentemente lidam com problema de palavras desconhecidas ou palavras fora do vocabulário (out-of-vocabulary). Os character embeddings tratam bem esse problema, pois uma palavra fora do vocabulário pode ser representada simplesmente como a composição individual de seus caracteres (26).

Character embeddings têm atraído a atenção de pesquisadores, Santos e Gatti (36) usaram a representação de character embedding para classificar sentimentos em textos. A representação de character embedding foi construída usando uma rede neural convolucional. Kim et el. (37) construíram uma rede neural que usa apenas a representação de character embedding. Eles conseguiram resultados competitivos no problema de modelagem de linguagem (prever a probabilidade de uma sequência de tokens pertencer a um idioma). 


\section{4}

\section{Trabalhos Relacionados}

Este capítulo apresenta os trabalhos relacionados mais relevantes sobre POS tagging, na seção 4.1 são apresentados os principais sistemas publicados destinados a Língua Portuguesa, e na seção 4.2 sistemas destinados a outras línguas.

\section{1}

\section{POS tagging para Português}

No ano 2000 Finger (61) apresentou um POS tagger para Português baseado em regras que foi construído usando o Corpus Tycho Brahe de Português Histórico. Finger reportou uma acurácia de 95.43\%.

"Basicamente, trata-se de um etiquetador baseado em regras transformacionais, isto é, regras que sucessivamente transformam uma etiquetagem inicial. Tais regras são aprendidas na fase de treinamento a partir de um corpus etiquetado manualmente. Este etiquetador baseia-se em um método proposto por Brill (62) (63). Um exemplo de tal regra seria: Mude a etiqueta da palavra corrente de VB (verbo) para $\mathrm{N}$ (nome) se a etiqueta da palavra anterior é D (determinante)." (Finger, 2000) (61)

Em (64) no ano de 2006, os autores propuseram um POS tagger para o Português. O etiquetador foi construído usando cadeias de Markov de alcance variável. Eles também usaram o corpus Tycho Brahe de Português Histórico e obtiveram $95.51 \%$ de acurácia na avaliação.

Em 2008 Santos, Milidiú e Rentería (65) aplicaram o Entropy Guided Transformation Learning (ETL) para resolver o problema de POS tagging. O algoritmo ETL consistem em combinar o uso de uma árvore de decisão com o algoritmo de classificação baseado em regras transformacionais de Brill (63). Os autores realizaram os experimentos usando o corpus Mac-Morpho v1 e o corpus Tycho Brahae, reportando uma acurácia de $96.75 \%$ e $96.64 \%$ para Mac-Morpho v1 e Tycho Brahae, respectivamente.

Fonseca e Rosa em 2013 (48), propuseram uma nova versão do corpus Mac-Morpho. Eles removeram erros do corpus, realizaram limpeza nos dados, juntaram algumas etiquetas. Os autores então treinaram um POS tagger 
usando uma rede neural perceptron multicamadas. Eles reportaram uma acurácia de 96.48\% para o Mac-Morpho revisado (v2).

Em (43) no ano de 2014, os autores usaram um perceptron estruturado de margem máxima para resolver o problema de POS tagging. Eles usaram um pequeno conjunto de features feitas à mão para melhorar o desempenho da aplicação. O classificador foi testado em duas corpora em Português: a versão original do Mac-Morpho corpus (v1) e na versão revisada do Mac-Morpho corpus (v2). Eles obtiveram o melhor resultado da época em acurácia no MacMorpho v1 (97.18\%) e resultados bem competitivos com o Mac-Morpho v2 $(97.25 \%)$.

O modelo proposto por Dos Santos e Zadrozny em 2014 (44) para resolver POS tagging foi construído sem nenhum tipo de features feitas à mão. Eles usaram uma arquitetura de rede neural que combina vetores de word embeddings com vetores de character embeddings para representar as palavras. Os vetores de word embeddings foram pré-treinados usando Word2Vec, e os character embeddings foram gerados a partir de camada de rede neural convolucional. Os autores avaliaram a arquitetura de rede em três corpora em Português: Mac-Morpho v1, Mac-Morpho v2 e Tycho Brahe corpus. E reportaram acurácia de 97.47\%, 97.31\% e 97.17\% para Mac-Morpho v1, MacMorpho v2 e Tycho Brahae, respectivamente.

Em 2015, Fonseca et al. (45) usaram uma rede perceptron multicamadas para o POS tagging. Essa rede usa vetores de word embeddings e algumas features manuais. Os autores apresentaram uma nova versão do corpus MacMorpho, com a correção de alguns erros e a junção de algumas classes. E ainda, compararam diferentes formas de gerar a representação de word embeddings. Fonseca et al (45) reportaram o estado da arte para três corpora em Português. Com $97.57 \%$ de acurácia no Mac-Morpho v1 corpus, 97.48\% no Mac-Morpho v2, e $97.33 \%$ no Mac-Morpho v3.

Na Tabela 4.1 temos um resumo dos trabalhos relacionados apresentados nessa seção. Na tabela marcamos se o trabalho usou features manuais, vetores de word embeddings ou vetores character embeddings. Ainda apresentamos quais algoritmos foram usados e quais os resultados reportados em acurácia. 
Tabela 4.1: Trabalhos relacionados para POS tagging em Português

\begin{tabular}{l|c|c|c|l|l} 
& \multicolumn{3}{|c|}{ Features } & & \\
\hline & Manual & Word e. & Char. e. & Algoritmos & Avaliação (Ac. \%) \\
\hline Finger, 2000 & $\mathrm{x}$ & & & Regras Transformacionais & Tycho Brahe: 95.43 \\
\hline Kepler e Finger, 2006 & $\mathrm{x}$ & & & Cadeias de Markov & Tycho Brahe: 95.51 \\
\hline $\begin{array}{l}\text { Santos, Milidiú e Rentería, } \\
\text { 2008 }\end{array}$ & $\mathrm{x}$ & & & $\begin{array}{l}\text { Regras Transformacionais/ } \\
\text { Árvore de Decisão }\end{array}$ & $\begin{array}{l}\text { Tycho Brahe: 96.64 } \\
\text { Mac-Morpho v1: 96.75 }\end{array}$ \\
\hline Fonseca e Rosa, 2013 & $\mathrm{x}$ & & & Perceptron Multicamadas & Mac-Morpho v2: 96.48 \\
\hline Fernandes et al, 2014 & $\mathrm{x}$ & & & $\begin{array}{l}\text { Perceptron Estruturado } \\
\text { de Margem Máxima }\end{array}$ & $\begin{array}{l}\text { Mac-Morpho v1: 97,18 } \\
\text { Mac-Morpho v2: 97.25 }\end{array}$ \\
\hline Santos e Zadrozny, 2014 & & $\mathrm{x}$ & $\mathrm{x}$ & $\begin{array}{l}\text { CNN/ } \\
\text { Algoritmo de Viterbi }\end{array}$ & $\begin{array}{l}\text { Mac-Morpho v1: 97.47 } \\
\text { Mac-Morpho v2: 97.3 } \\
\text { Tycho Brahe: 97.17 }\end{array}$ \\
\hline Fonseca et al, 2015 & $\mathrm{x}$ & $\mathrm{x}$ & & Perceptron Multicamadas & $\begin{array}{l}\text { Mac-Morpho v1: 97.57 } \\
\text { Mac-Morpho v2: 97.48 } \\
\text { Tycho Brahe: 96.91 }\end{array}$ \\
\hline
\end{tabular}

\section{2}

\section{POS tagging para outros idiomas}

Giménez e Marquez (13) apresentaram em 2004 uma ferramenta para POS tagging construída usando o algoritmo de máquina de vetores de suporte. Eles aplicaram um rico conjunto de features manuais no processo de aprendizagem e conseguiram resultados competitivos para inglês no corpus Wall Street Journal e para espanhol no corpus LEXESP.

Em (25) os autores propuseram uma arquitetura de rede neural para rotulagem de sequência sem usar features manuais. Primeiro, uma camada de rede neural convolucional foi usada para extrair uma representação de character embeddings. E então, essa representação foi concatenada com informações de word embeddings para ser usada em uma camada de BLSTM. E, em cima da camada de BLSTM, foi usado uma camada de CRF.

A arquitetura de (25) foi testada em duas atividades de rotulagem de sequência: em POS tagging com o corpus Wall Street Journal e em reconhecimento de entidade nomeada (REN) com o corpus CoNLL 2003. Eles reportaram estado da arte em acurácia para POS tagging e REN.

Em 2018 (46) Marulli et al. usaram uma arquitetura de rede neural para encarar o problema. Eles aplicaram uma camada de BLSTM para extrair as informações de character embeddings e então concatenaram com informações de word embeddings para então alimentar uma outra camada de BLSTM com essas informações. A arquitetura foi avaliada usando um corpus em Italiano. Os autores reportaram uma acurácia de 98.02\%. Eles ainda avaliaram o desempenho do word2vec e FastText, dois algoritmos para calcular word embeddings. O modelo que usou o FastText teve um melhor desempenho em acurácia geral e acurácia das palavras fora do vocabulário.

Bohnet et al. (1) reportaram o atual estado da arte para POS Tagging na Língua Inglesa. Eles usam o corpus Penn Treebank WSJ e atingiram 97.96\% de 
acurácia. Os bons resultados são obtidos com a integração das representações vetoriais de palavra e de caracteres. Um meta-modelo aprende como combinar essas duas representações através de um treinamento sincronizado.

Na Tabela 4.2 temos um resumo dos trabalhos relacionados apresentados nessa seção. Na tabela marcamos se o trabalho usou features manuais, vetores de word embeddings ou vetores character embeddings. Ainda apresentamos quais algoritmos foram usados e quais os resultados reportados em acurácia.

Tabela 4.2: Trabalhos relacionados para POS tagging em outros idiomas

\begin{tabular}{|c|c|c|c|c|c|}
\hline \multicolumn{6}{|c|}{ Features } \\
\hline & Manual & Word e. & Char. e. & Algoritmos & Avaliação (Ac. \%) \\
\hline $\begin{array}{l}\text { Giménez e Marquez, } \\
2004\end{array}$ & $\mathrm{x}$ & & & SVM & $\begin{array}{l}\text { WSJ: } 97.16 \\
\text { LEXESP: } 96.89\end{array}$ \\
\hline Ma e Hovy, 2016 & & $\mathrm{x}$ & $\mathrm{x}$ & $\begin{array}{l}\text { CNN / } \\
\text { BLSTM / } \\
\text { CRF }\end{array}$ & WSJ: 97.55 \\
\hline Marulli et al., 2018 & & $\mathrm{x}$ & $\mathrm{x}$ & $\begin{array}{l}\text { BLSTM / } \\
\text { BLSTM }\end{array}$ & UD V2.1 It: 98.00 \\
\hline Bohnet et al., 2018 & & $\mathrm{x}$ & $\mathrm{x}$ & Meta BiLSTM & WSJ: 97.96 \\
\hline
\end{tabular}




\section{Metodologia}

Esse trabalho se propõe construir um POS tagging para a Língua Portuguesa usando redes neurais recorrentes que já se estabeleceram como estado da arte para o POS tagging em outros idiomas. Nessa abordagem não usamos features construídas manualmente ou informações externas ao conjunto de dados. A arquitetura é testada em três datasets que abrangem o Português Contemporâneo e o Português Histórico.

\section{1}

\section{Arquitetura de Rede Neural}

A arquitetura usada nesse trabalho é inspirada nos trabalhos de Ma e Hovy de 2016 (25) e Marulli et al. de 2018 (46). Na Figura 6.9, temos a ilustração da arquitetura de rede usada para treinar o modelo de $P O S$ tagging. Seja a sentença $S$, decomposta em $n$ tokens $t_{1}, t_{2}, \ldots, t_{n}$ com suas $n$ etiquetas correspondentes $e_{1}, e_{2}, \ldots, e_{n}$, nós treinamos uma camada de BLSTM para predizer a probabilidade de um token pertencer a cada etiqueta. Com isso atribuímos ao token $t_{x}$ a etiqueta $e_{y}$ de maior probabilidade associada.

Para montar a arquitetura ilustrada na Figura 5.1, é preciso representar cada token da sentença como um vetor no espaço $R^{d}$, esse vetor de representação é resultado da concatenação da representação de word embeddings e da representação de character embeddings. Com isso, é possível capturar das palavras informações sintáticas e semânticas (word embeddings) e informações morfológicas (character embeddings).

A representação de word embeddings para um token $t_{x}$ é fornecida por um modelo, já pré-treinado em grandes corpora não supervisionados. A representação de character embeddings para um token $t_{x}$ é gerada por uma outra camada de BLSTM. Mais formalmente, como ilustrado na Figura 5.2, a entrada para a camada de BLSTM é um único token $t_{x}$, e a saída é um vetor $v$-dimensional de character embeddings.

Para gerar a representação de character embeddings, é criado um vocabulário $V$ de caracteres que contêm todas a letras maiúsculas, minúsculas e também números e pontuações presentes no corpus de treino. Um token $t_{x}$ de entrada é decomposto em uma sequência de $m$ caracteres $c_{1}, c_{2}, \ldots, c_{m}$, onde $m$ 
representa o tamanho do token $t_{x}$. Cada caracteres $c_{i}$ é representado como um vetor de zeros $1_{c i}$, com o valor 1 presente no índice que representa o caractere $c_{i}$ no vocabulário $V$. Finalmente, a representação do token $t_{x}$ é definida como a concatenação dos estados de forward e backward provenientes da camada de BLSTM.

Com os tokens devidamente representados, as representações de word embeddings e character embeddings são concatenadas e então passadas para a camada principal de BLSTM. Uma função de ativação Softmax é usada no topo da arquitetura, para gerar as probabilidades para as etiquetas.

Durante o treino dessa arquitetura usamos a função de erro sparse categorical crossentropy. Essa função compara os valores preditos pela a rede neural, com os valores de classes reais para calcula a perda. A função sparse categorical crossentropy é geralmente usada em problemas de classificação em que as classes são mutualmente exclusivas (cada exemplo só pode assumir uma classe por vez). Na Equação 5-1 temos a definição desse função, onde $S$ representa o conjunto de exemplos e $C$ o conjunto de classes possíveis. O $\log (s \in c)$ representa a probabilidade predita pelo modelo do $s$-ésimo exemplo pertencer a $c$-ésimo classe.

$$
-\frac{1}{N} \sum_{s \in S} \sum_{c \in C} 1_{s \in c} \log (s \in c)
$$



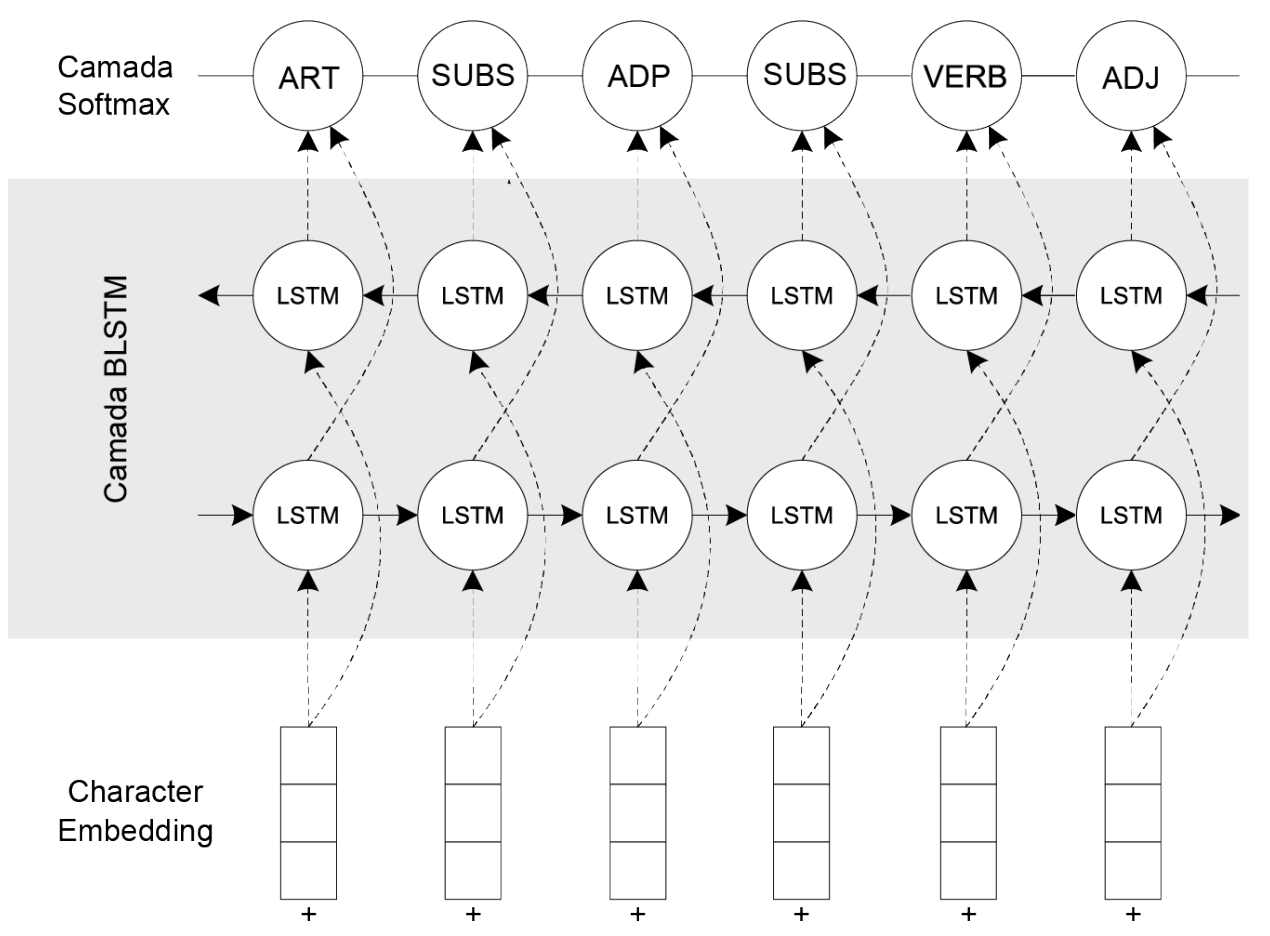

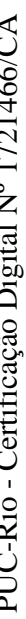
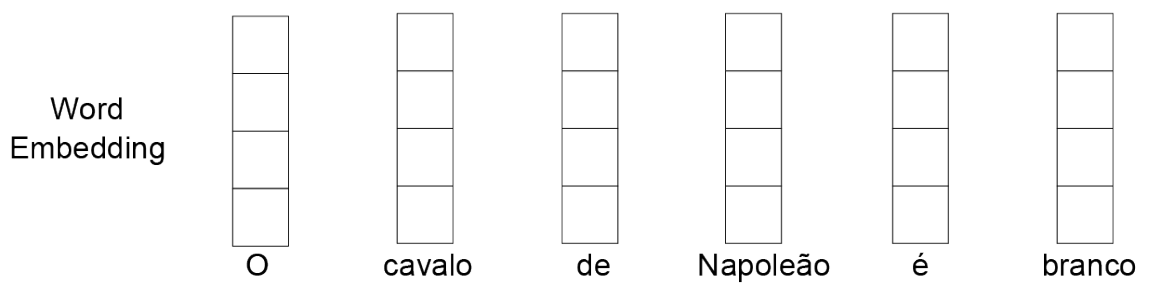

Figura 5.1: Modelo BLSTM-WE-CE. Arquitetura da rede neural para $P O S$ tagging.

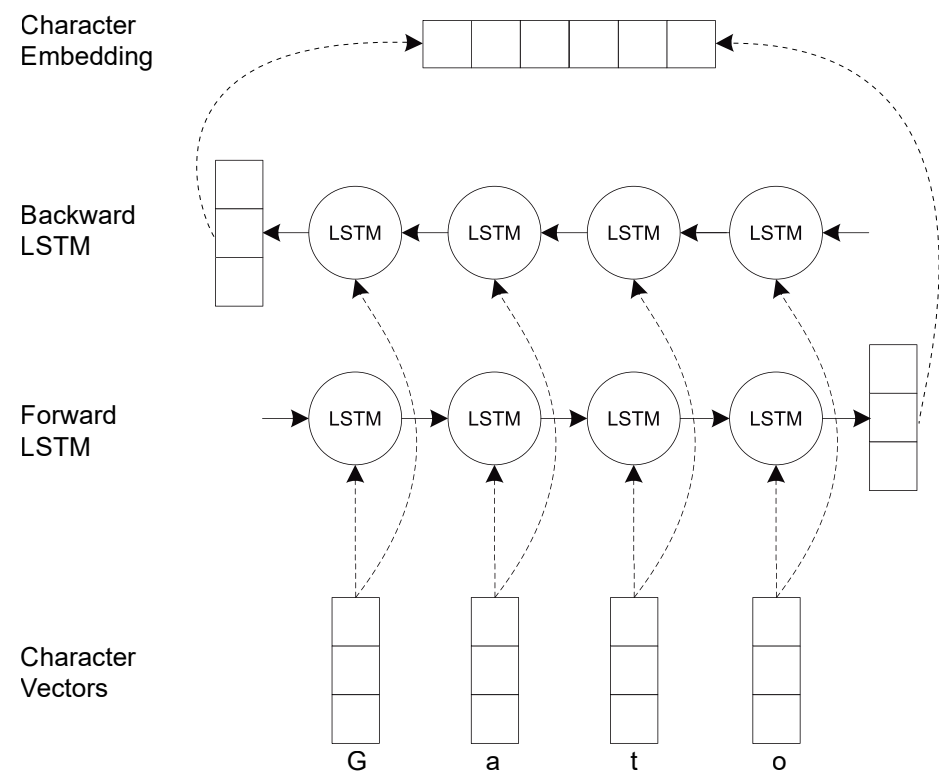

Figura 5.2: Arquitetura do character embeddings 


\section{6 \\ Experimentos}

Neste capítulo descrevemos todos os experimentos realizados, os corpora utilizados e as métricas de avaliação. Usamos os corpora Mac-Morpho v1, Mac-Morpho v2 e Tycho Brahe, todos com as mesmas partições de treino/teste/validação usadas nos trabalhos de Dos Santos e Zadrozny (44) e Fonseca et al. (45). O código dos modelos foram escritos na linguagem Python ${ }^{1}$, usando Keras ${ }^{2}$, uma biblioteca para rede neural de alto nível. Usamos TensorFlow $^{3}$ como backend com a biblioteca Keras. Todos os experimentos foram realizados usando um GPU GeForce GTX TITAN X. As arquiteturas de redes neurais descritas nesse Capítulo foram treinadas usando a otimizador "adam" (68) com 5 épocas no treino.

\section{1 \\ Corpora}

Nós avaliamos o modelo de rede neural em três corpora diferentes: a versão original do Mac-Morpho (47), a versão revisada do Mac-Morpho (48) e o Tycho Brahe (49) corpus. Na Tabela 6.1, mostramos a quantidade de sentenças, tokens e classes dos corpora. Usamos as mesmas partições de treino/teste/desenvolvimento que (44) e (45) para comparar os resultados diretamente.

Tabela 6.1: Partições dos Corpora

\begin{tabular}{llrrrrr}
\hline Corpus & & Treino & Validação & Teste & Total & Classes \\
\hline \multirow{2}{*}{ Mac-Morpho v1 } & Sentences & 42,022 & 2,211 & 9,141 & 53,374 & \multirow{2}{*}{41} \\
& Tokens & 957,439 & 50,232 & 213,794 & $1,221,465$ & \\
\hline \multirow{2}{*}{ Mac-Morpho v2 } & Sentences & 42,742 & 2,249 & 4,999 & 49,990 & \multirow{2}{*}{30} \\
& Tokens & 807,796 & 43,141 & 94,991 & 945,928 & \\
\hline \multirow{2}{*}{ Tycho Brahe } & Sentences & 29,162 & 1,534 & 10,233 & 40,929 & \multirow{2}{*}{265} \\
& Tokens & 734,889 & 40,673 & 259,913 & $1,035,475$ & \\
\hline
\end{tabular}

\footnotetext{
${ }^{1}$ https://www.python.org/

${ }^{2}$ https://keras.io/

${ }^{3}$ https://www.tensorflow.org/
} 


\subsection{1}

\section{Mac-Morpho v1}

Mac-Morpho é um corpus composto por textos em Português do Brasil com anotação morfossintática dos tokens. Os textos foram extraídos de uma coleção textual da Folha de São Paulo ${ }^{4}$, que implica num texto contemporâneo de alta qualidade e diferentes autores e domínios (47). O corpus conta com 53.374 sentenças, 1.221 .465 tokens e 41 classes no total. Na Tabela 6.2 temos a descrição das etiquetas presentes no corpus e alguns exemplos de tokens presentes.

Na Tabela 6.3 apresentamos a distribuição dos tokens por classe, mostrando a quantidade de exemplos presente nos conjuntos de treino, validação e teste. É possível observar que substantivos (N), preposição (PRED), artigos (ART), substantivos próprio (NPROP) e verbos (V) são as etiquetas mais frequentes. Algumas etiquetas de pontuação não possuem representações no conjunto de validação ou no conjunto de teste, isso torna impossível avaliar o desempenho do modelo para essas classes. Para melhor comparação entre valor, na Figura 6.1, apresentamos a distribuição total das etiquetas mais frequentes no corpus Mac-Morpho v1.

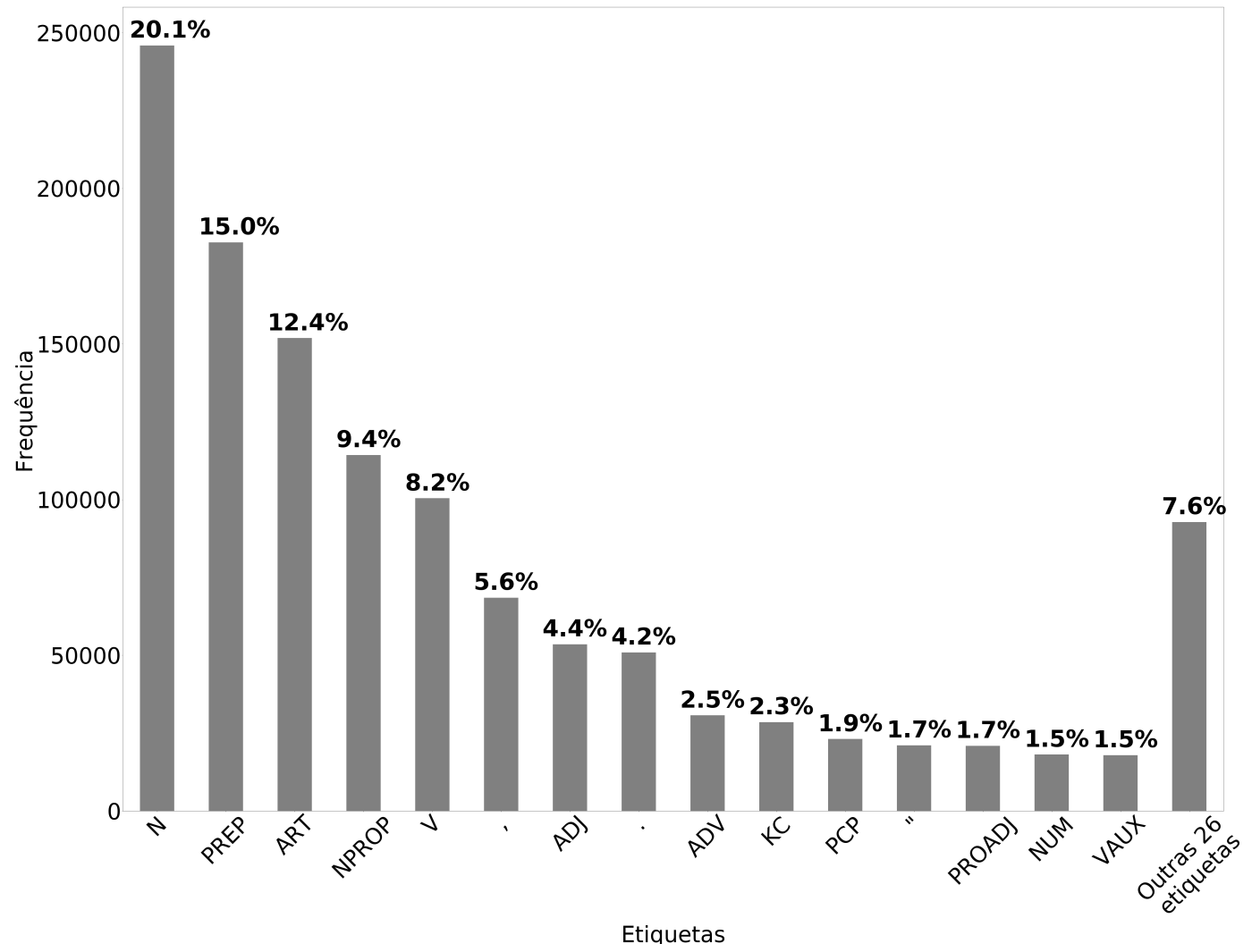

Figura 6.1: Distribuição das etiquetas no Mac-Morpho v1

${ }^{4}$ http://www.folha.uol.com.br/folha 
Tabela 6.2: Descrição do corpus Mac-Morpho v1

\begin{tabular}{|c|c|c|}
\hline Etiqueta & Significado & Exemplos \\
\hline $\mathrm{N}$ & Substantivo & dia, governo, presidente, milhões, país \\
\hline PREP & Preposição & de, em, a, para, por \\
\hline ART & Artigo & $o, a$, as, as, um \\
\hline NPROP & Substantivos próprio & paulo, são, brasil, eua, rio \\
\hline $\mathrm{V}$ & Verbo & é, foi, tem, disse, são \\
\hline , & Pontuação & 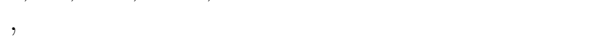 \\
\hline \multirow{2}{*}{ ADJ } & Adjetivo & maior, grande, primeiro, novo, nova \\
\hline & Pontuação & . \\
\hline $\mathrm{ADV}$ & Advérbio & não, mais, amanhã, basicamente, principalmente \\
\hline $\mathrm{KC}$ & Conjunção coordenativa & e, mas, ou, pois, porém \\
\hline PCP & Particípio & passado, sido, feito, usado, conhecido \\
\hline$"$ & Pontuação & " \\
\hline PROADJ & Pronome adjetivo & sua, seu, este, seus, esse \\
\hline NUM & Numeral & 100, 455, dois, mil, um \\
\hline VAUX & Verbo auxiliar & foi, ser, pode, vai, é \\
\hline PROPESS & Pronome pessoal & se, ele, eu, eles, ela \\
\hline $\mathrm{KS}$ & Conjunções Subordinativas & que, se, quando, como, porque \\
\hline PRO-KS-REL & Subordinação do pronome relativo conectivo & que, qual, o, a quais \\
\hline PROSUB & Pronome nominal & isso, o, um, uma, tudo \\
\hline ) & Pontuação & ) \\
\hline( & Pontuação & ( \\
\hline PDEN & Palavra denotativa & também, só, apenas, mesmo, até \\
\hline$:$ & Pontuação & $:$ \\
\hline- & Pontuação & - \\
\hline CUR & Símbolo de moeda & $\mathrm{us}, r, \mathrm{cr}, r s, \mathrm{u} \$$ \\
\hline PRO-KS & Pronome conectivo de subordinação & que, o, quem, qual, quanto \\
\hline$?$ & Pontuação & $?$ \\
\hline ; & Pontuação & ; \\
\hline ! & Pontuação & ! \\
\hline ADV-KS-REL & Adjetivo relativo de subordinação & onde, quando, que, como, aonde \\
\hline \multirow[t]{2}{*}{ - } & Pontuação & .. \\
\hline & Pontuação & \\
\hline IN & Interjeição & ora, ah, não, ô, oh \\
\hline ADV-KS & Advérbio conectivo de subordinação & como, onde, quando, por, que \\
\hline / & Pontuação & / \\
\hline$\$$ & Pontuação & $\$$ \\
\hline[ & Pontuação & {[} \\
\hline igual & Pontuação & igual \\
\hline$)$ ) & Pontuação & )$)$ \\
\hline$(($ & Pontuação & $(($ \\
\hline 6 & Pontuação & ! \\
\hline
\end{tabular}

Por conta da ambiguidade presente na linguagem natural, uma única palavra pode assumir diversas classes morfossintáticas dependendo do contexto em que é usada. Por exemplo, na sentença: "Vamos tomar banho de rio?", a palavra "rio" é usada como substantivo (rio = curso de água fluvial). Na sentença: "Eu rio de suas piadas porque você é hilariante!", a palavra "rio" é usada como um verbo (rio = verbo rir na $1 .^{a}$ pessoa do singular).

Na Figura 6.2 temos as 15 palavras do Mac-Morpho v1 com o maior número de classes morfossintáticas atribuídas. A palavra "que" foi rotulada com 17 etiquetas diferentes ao longo do dataset, a palavra "a", "o" e "como" foram associadas com 14, 13 e 12 etiquetas diferentes respectivamente ao longo do dataset. Palavras com muitas classes associadas podem ser mais difíceis de classificar.

A Figura 6.3 apresenta uma nuvem de palavras feita com corpus MacMorpho v1. Com essa visualização podemos perceber que palavras como "de", 
Tabela 6.3: Distribuição das etiquetas no corpus Mac-Morpho v1

\begin{tabular}{|c|c|c|c|c|}
\hline Etiqueta & Treino & Desen. & Teste & Total \\
\hline $\mathrm{N}$ & 192498 & 10117 & 43323 & $245938(20.13 \%)$ \\
\hline PREP & 142937 & 7464 & 32278 & $182679(14.96 \%)$ \\
\hline ART & 118730 & 6223 & 26949 & $151902(12.44 \%)$ \\
\hline NPROP & 88149 & 4691 & 21522 & $114362(9.36 \%)$ \\
\hline $\mathrm{V}$ & 79254 & 4214 & 16967 & $100435(8.22 \%)$ \\
\hline & 53628 & 2894 & 11972 & $68494(5.61 \%)$ \\
\hline ADJ & 42403 & 2169 & 8985 & $53557(4.38 \%)$ \\
\hline . & 39880 & 2080 & 8960 & $50920(4.17 \%)$ \\
\hline $\mathrm{ADV}$ & 24493 & 1322 & 4895 & $30710(2.51 \%)$ \\
\hline $\mathrm{KC}$ & 22468 & 1173 & 4901 & $28542(2.34 \%)$ \\
\hline $\mathrm{PCP}$ & 18083 & 861 & 4148 & $23092(1.89 \%)$ \\
\hline " & 16305 & 888 & 3876 & $21069(1.72 \%)$ \\
\hline PROADJ & 16621 & 854 & 3446 & $20921(1.71 \%)$ \\
\hline NUM & 13992 & 749 & 3371 & $18112(1.48 \%)$ \\
\hline VAUX & 13908 & 692 & 3332 & $17932(1.47 \%)$ \\
\hline PROPESS & 11889 & 610 & 2223 & $14722(1.21 \%)$ \\
\hline $\mathrm{KS}$ & 11380 & 591 & 2358 & $14329(1.17 \%)$ \\
\hline PRO-KS-REL & 8928 & 436 & 1983 & $11347(0.93 \%)$ \\
\hline PROSUB & 6987 & 368 & 1243 & $8598(0.70 \%)$ \\
\hline ） & 6063 & 293 & 1385 & $7741(0.63 \%)$ \\
\hline( & 6032 & 296 & 1385 & $7713(0.63 \%)$ \\
\hline PDEN & 5282 & 297 & 1209 & $6788(0.56 \%)$ \\
\hline$:$ & 5511 & 304 & 921 & $6736(0.55 \%)$ \\
\hline- & 2485 & 120 & 512 & $3117(0.26 \%)$ \\
\hline CUR & 2118 & 103 & 485 & $2706(0.22 \%)$ \\
\hline PRO-KS & 1686 & 80 & 384 & $2150(0.18 \%)$ \\
\hline$?$ & 1259 & 85 & 166 & $1510(0.12 \%)$ \\
\hline ; & 1120 & 68 & 188 & $1376(0.11 \%)$ \\
\hline$!$ & 860 & 45 & 14 & $919(0.08 \%)$ \\
\hline ADV-KS-REL & 695 & 42 & 161 & $898(0.07 \%)$ \\
\hline$\ldots$ & 538 & 42 & 37 & $617(0.05 \%)$ \\
\hline , & 388 & 20 & 102 & $510(0.04 \%)$ \\
\hline IN & 380 & 16 & 20 & $416(0.03 \%)$ \\
\hline ADV-KS & 295 & 19 & 78 & $392(0.03 \%)$ \\
\hline / & 83 & 2 & 10 & $95(0.01 \%)$ \\
\hline$\$$ & 57 & 3 & 0 & $60(0.0 \%)$ \\
\hline[ & 18 & 0 & 5 & $23(0.0 \%)$ \\
\hline$=$ & 14 & 0 & 0 & $14(0.0 \%)$ \\
\hline )) & 10 & 0 & 0 & $10(0.0 \%)$ \\
\hline (( & 10 & 0 & 0 & $10(0.0 \%)$ \\
\hline ‘ & 2 & 1 & 0 & $3(0.0 \%)$ \\
\hline
\end{tabular}

"para", "que", "em" e "como" são muito frequentes no corpus. Também podemos perceber o tema jornalístico do corpus pela a grande frequência de termos como "disse que", "políticos", "brasil", "milhões de", "presidente de", 
entre outros.

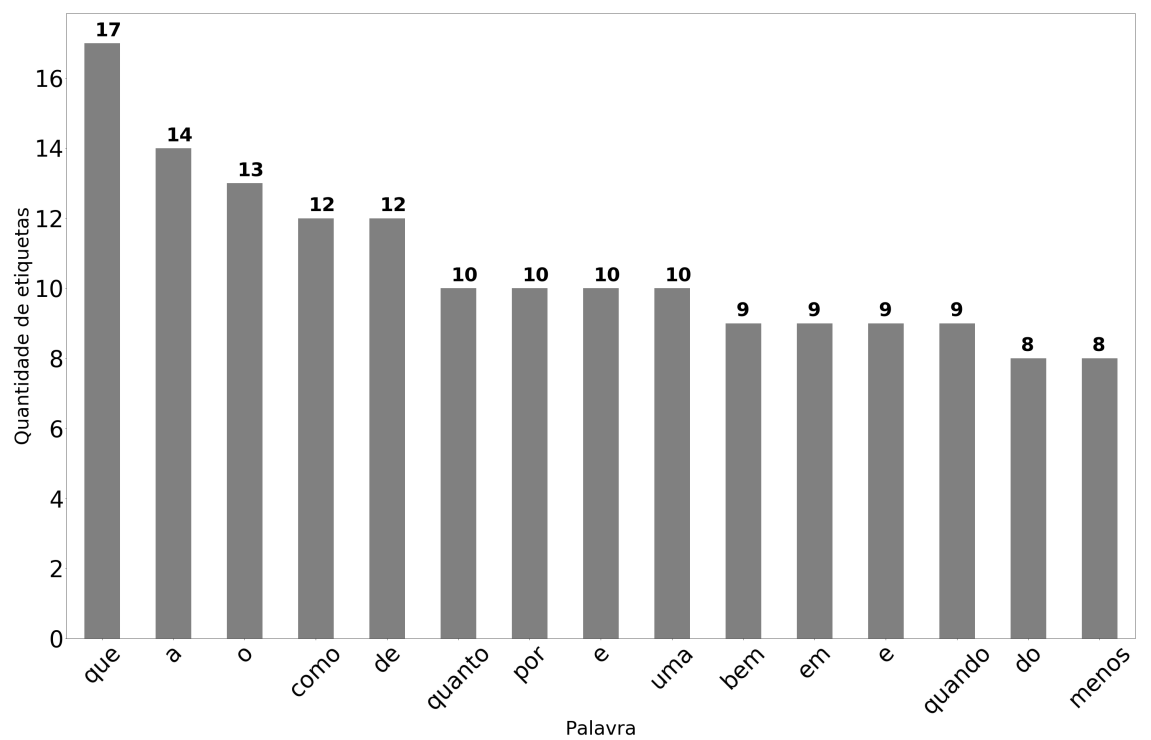

Figura 6.2: Distribuição das palavras com o maior número de diferentes etiquetas associadas. Corpus Mac-Morpho v1.

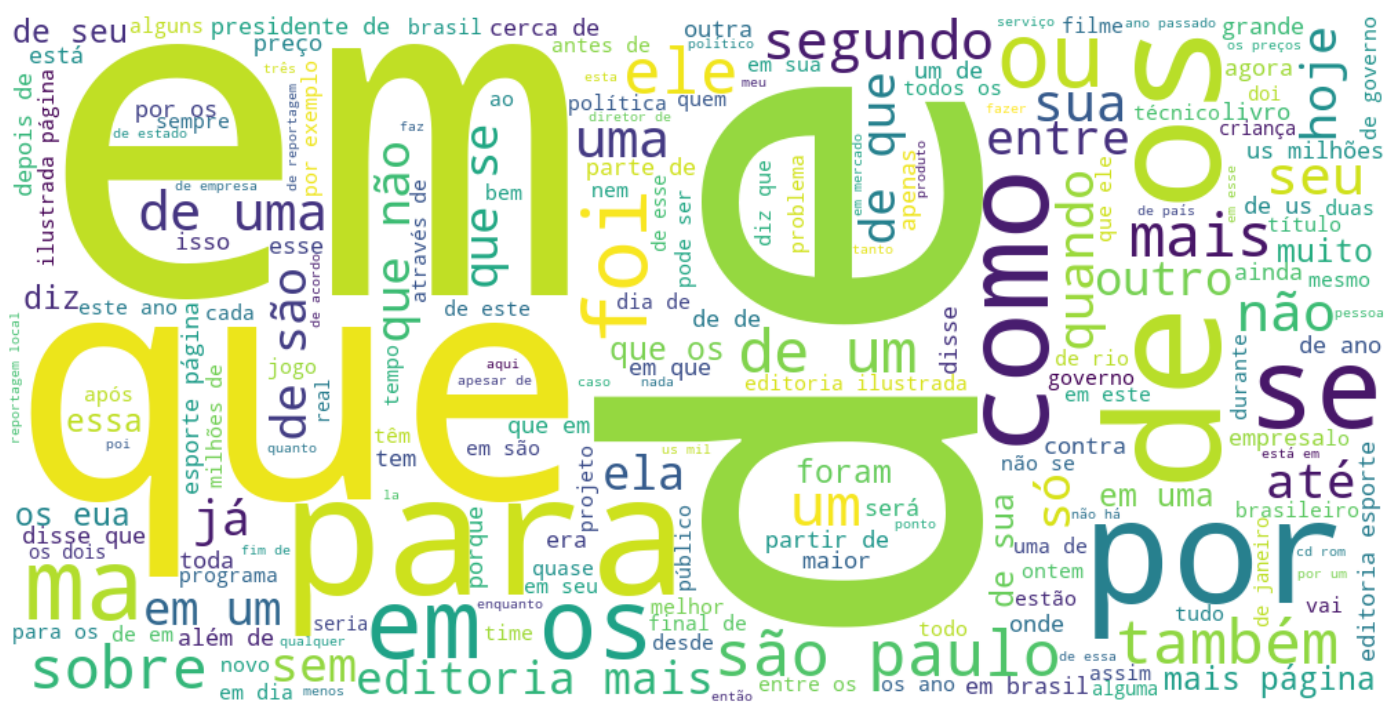

Figura 6.3: Nuvem de palavras Mac-Morpho v1

\section{1 .2}

\section{Mac-Morpho v2}

Mac-Morpho foi revisado em 2013 em (48). A revisão consiste em duas partes principais: limpeza de ruído nos dados e alteração do conjunto de tags para incluir contrações de preposições. Foram concatenadas formações como por exemplo: "de + o", "de + a" que apareciam separadas no corpus, a fim de refletir algo mais parecido com o cenário real. O Mac-Morpho (v2) Revisado 
contém 49.990 sentenças, 945,928 tokens e 30 classes no total. Na Tabela 6.4 temos as etiquetas presentes no Mac-Morpho v2, seus significados e alguns exemplo de etiquetas. Os toknes de pontuação foram reunidos em uma única etiqueta (PU).

Na Tabela 6.5 temos a distribuição das etiquetas nos conjuntos de treino, validação e treino. Substantivos $(\mathrm{N})$, pontuação (PU), substantivos próprio (NPROP), preposição (PRED), verbos (V) e artigos (ART), são as etiquetas mais frequentes no mac-morpho v2. Na Figure 6.4 apresentamos as etiquetas mais frequentes no corpus Mac-Morpho v2.

Também apresentamos a nuvem de palavras para o Mac-Morpho v2 na Figura 6.5. O conteúdo textual dos corpora Mac-Morpho v1 e Mac-Morpho v2 são essencialmente o mesmo, por isso a nuvem de palavras gerada para o Mac-Morpho v2 é muito similar a nuvem do Mac-Morpho v1. Com essa figura podemos ver o carácter jornalístico do corpus Mac-Morpho v2.

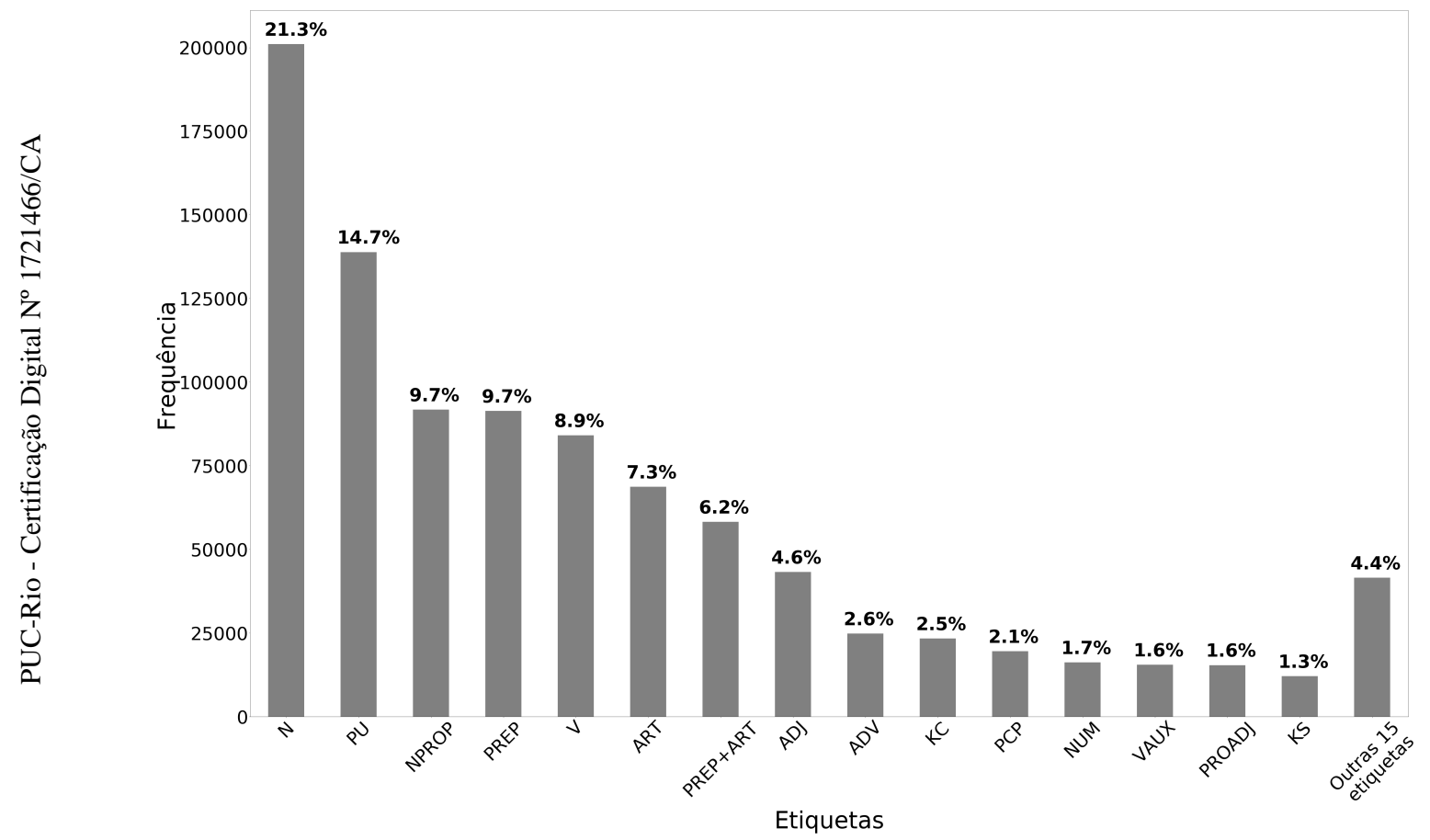

Figura 6.4: Distribuição das etiquetas no Mac-Morpho v2

\subsection{3}

\section{Corpus Tycho Brahe}

O corpus Tycho Brahe foi escrito com um Português Histórico composto por textos em prosa, escritos em Português por falantes nativos, nascidos entre 1550 e 1850. Nesse dataset podemos encontrar, por exemplo, sermões do Padre Antônio Vieira, cartas de D. João III e trechos de obras de José de Alencar. Isso implica em um estilo de escrita e vocabulário bem diferente dos que são 


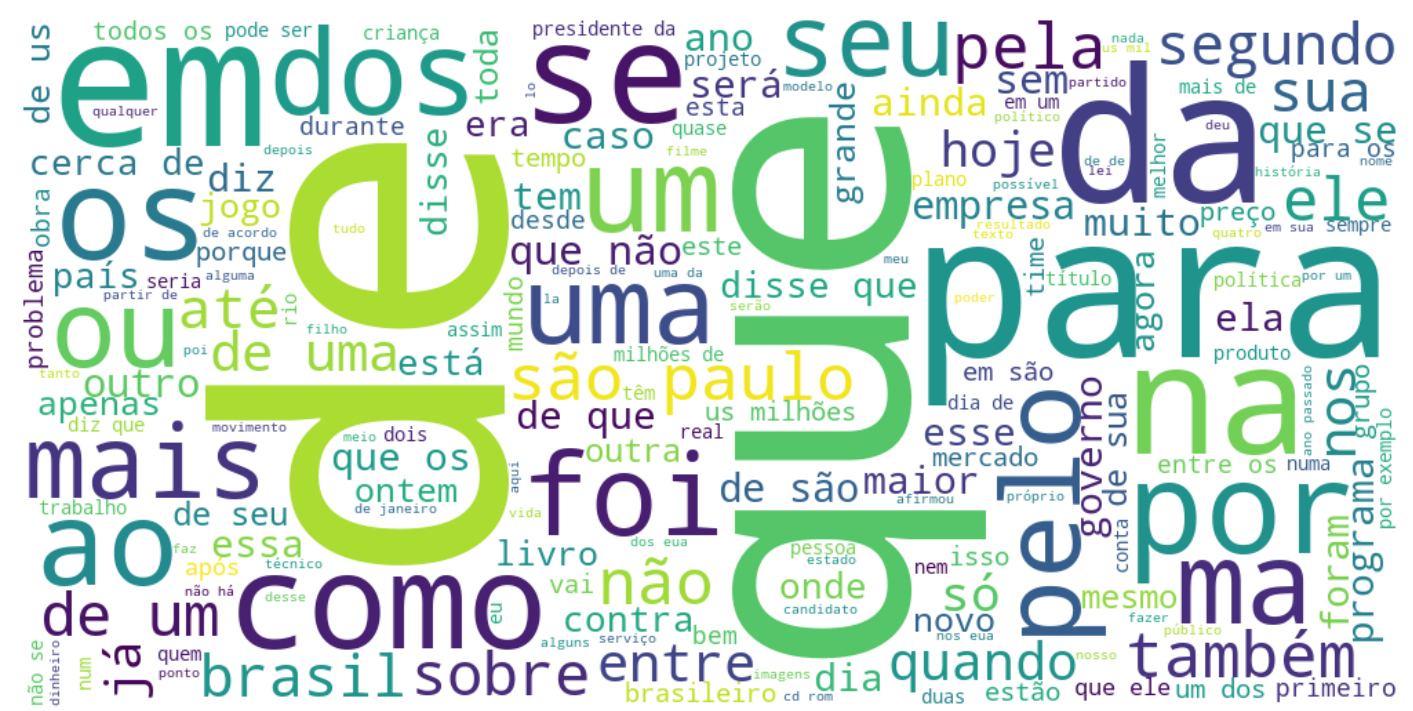

Figura 6.5: Nuvem de palavras Mac-Morpho v2

Tabela 6.4: Descrição do corpus Mac-Morpho v2

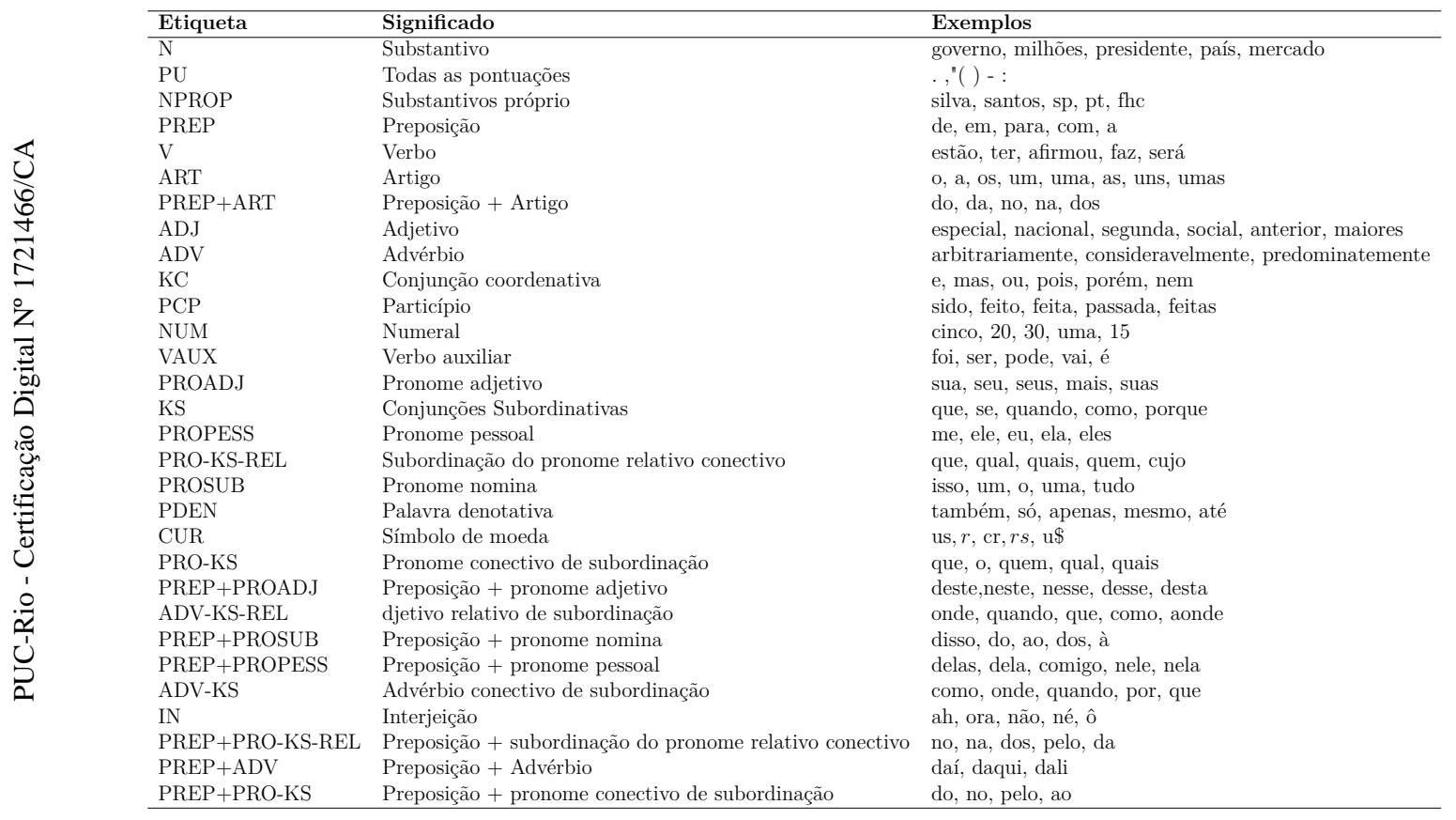

usados no Português Contemporâneo apresentado no Mac-Morpho v1 e no Mac-Morpho v2.

O corpus contém 40.929 sentenças, 1.035.475 tokens e 265 classes. Esse corpus possui uma quantidade muito maior de etiquetas pois inclui informações morfológicas como gênero, número e tempo verbal (49). Com esse conjunto de etiquetas podemos especificar, por exemplo, o tempo verbal empregado em uma palavra: verbo no infinitivo (VB), verbo no presente (VB-P) ou verbo no passado (VB-D). Também é possível distinguir entre forma e gênero de adjetivos: adjetivo masculino singular (ADJ-S), adjetivo feminino singular (ADJ-S-F), adjetivo masculino plural (ADJ-S-P) e adjetivo feminino plural 
Tabela 6.5: Distribuição das etiquetas no corpus Mac-Morpho v2

\begin{tabular}{llllr}
\hline Etiqueta & Treino & Desen. & Teste & Total \\
\hline N & 171690 & 9145 & 20181 & $201016(21.25 \%)$ \\
PU & 118548 & 6307 & 14021 & $138876(14.68 \%)$ \\
NPROP & 78345 & 4196 & 9237 & $91778(9.70 \%)$ \\
PREP & 77771 & 4332 & 9296 & $91399(9.66 \%)$ \\
V & 71874 & 3812 & 8415 & $84101(8.89 \%)$ \\
ART & 58865 & 3040 & 6804 & $68709(7.26 \%)$ \\
PREP+ART & 49927 & 2652 & 5680 & $58259(6.16 \%)$ \\
ADJ & 37055 & 1954 & 4264 & $43273(4.57 \%)$ \\
ADV & 21192 & 1114 & 2509 & $24815(2.62 \%)$ \\
KC & 19967 & 1067 & 2333 & $23367(2.47 \%)$ \\
PCP & 16702 & 921 & 1927 & $19550(2.07 \%)$ \\
NUM & 13708 & 798 & 1692 & $16198(1.71 \%)$ \\
VAUX & 13265 & 704 & 1552 & $15521(1.64 \%)$ \\
PROADJ & 13098 & 669 & 1647 & $15414(1.63 \%)$ \\
KS & 10296 & 520 & 1275 & $12091(1.28 \%)$ \\
PROPESS & 9804 & 504 & 1228 & $11536(1.22 \%)$ \\
PRO-KS-REL & 7891 & 407 & 863 & $9161(0.97 \%)$ \\
PROSUB & 5401 & 309 & 672 & $6382(0.67 \%)$ \\
PDEN & 4858 & 262 & 546 & $5666(0.60 \%)$ \\
CUR & 2106 & 129 & 239 & $2474(0.26 \%)$ \\
PRO-KS & 1512 & 82 & 165 & $1759(0.19 \%)$ \\
PREP+PROADJ & 1474 & 75 & 166 & $1715(0.18 \%)$ \\
ADV-KS-REL & 613 & 35 & 69 & $717(0.08 \%)$ \\
PREP+PROSUB & 608 & 30 & 72 & $710(0.08 \%)$ \\
PREP+PROPESS & 452 & 27 & 54 & $533(0.06 \%)$ \\
ADV-KS & 277 & 12 & 31 & $320(0.03 \%)$ \\
IN & 242 & 25 & 17 & $284(0.03 \%)$ \\
PREP+PRO-KS-REL & 163 & 5 & 19 & $187(0.02 \%)$ \\
PREP+ADV & 66 & 6 & 13 & $85(0.01 \%)$ \\
PREP+PRO-KS & 26 & 2 & 4 & $32(0.00 \%)$ \\
\hline & & & & \\
& & $52 \%$ &
\end{tabular}

(ADJ-S-F-P).

Na Figura 6.6, apresentamos o número de exemplos por etiqueta no corpus Tycho Brahe. As etiquetas substantivo singular (N), Virgula (,) e preposição (P) são as etiquetas com mais exemplos em todo o corpus. A descrição e distribuição das 265 etiquetas do corpus Tycho Brahe são detalhadamente apresentadas no Apêndice A.

Na Figura 6.7 apresentamos a nuvem de palavras do Tycho Brahe Corpus, podemos observar a alta frequência de palavras como "que", "em", "para" , "se". Também podemos notar a presença de expressões como "vossa mercê", "vossa senhoria" e "de vossa" que indica o carácter histórico do português usado no corpus. 


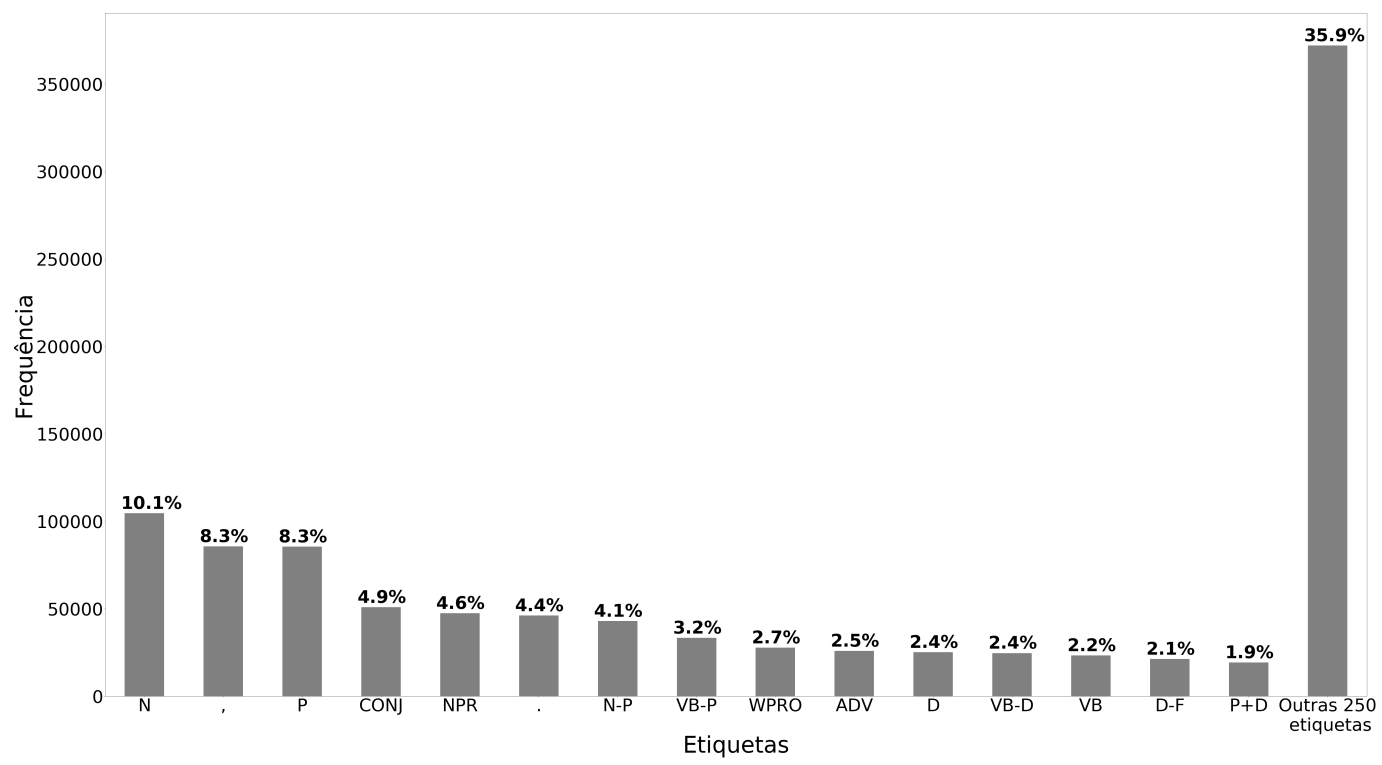

Figura 6.6: Distribuição das etiquetas no Tycho Brahe

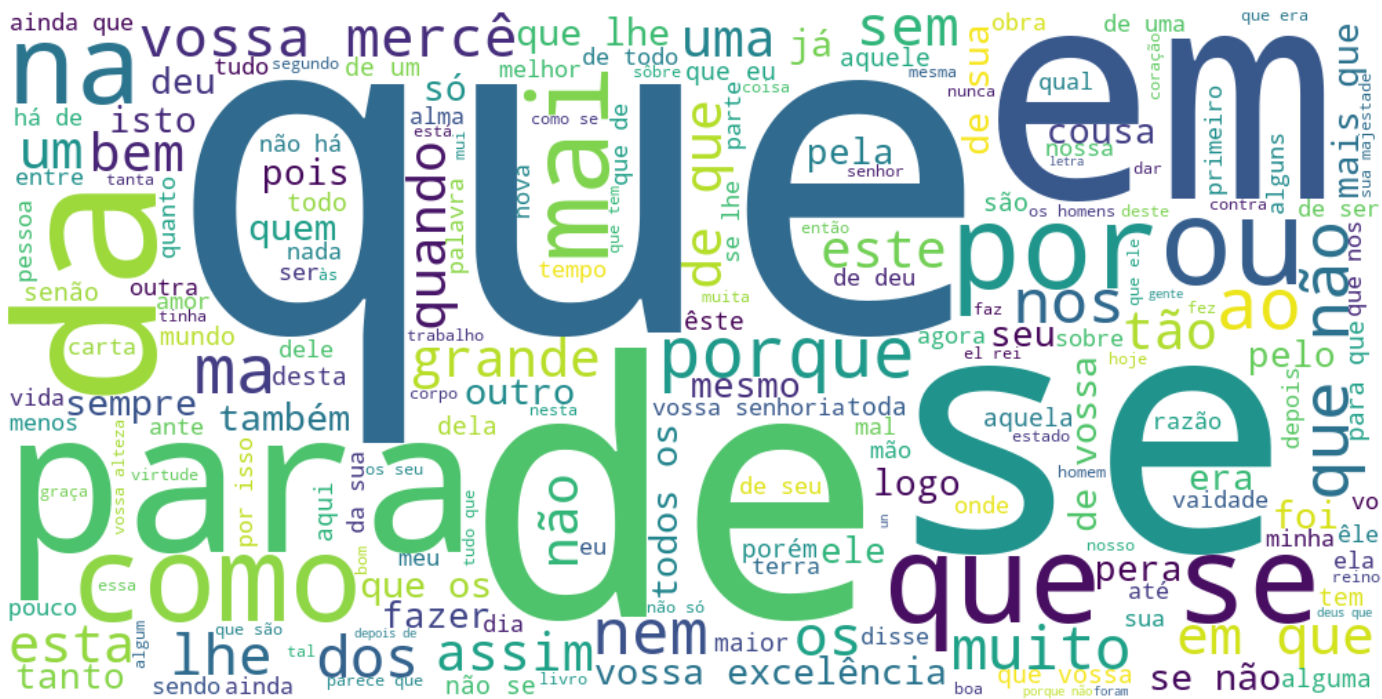

Figura 6.7: Nuvem de palavras Tycho Brahe 


\section{2}

\section{Métricas de avaliação}

Para avaliar o desempenho do modelo nós usamos acurácia, formalizada na equação 6-1. Outra métrica de avaliação importante é a acurácia de palavras fora do vocabulário (FV) que calcula uma acurácia local focada apenas em tokens não conhecidos pelo conjunto de treino. A acurácia FV captura o desempenho do modelo em palavras desconhecidas, essa métrica é formalizada na equação 6-2. Nós também calculamos precision, recall e $F_{1}$-score (conhecido também como $F_{1}$ ) para cada classe $i$ presente no corpus. As equações 6-3, 6-4 e 6-6 definem precision, recall e $F_{1}$.

$$
\begin{aligned}
& \text { Acurácia }=\frac{\# \text { de tokens corretamente classificados }}{\# \text { total de tokens }} \\
& \text { Acurácia } F V=\frac{\# \text { de tokens } F V \text { corretamente classificados }}{\# \text { total de tokens } F V} \\
& \text { Precision }_{i}=\frac{T P_{i}}{T P_{i}+F P_{i}} \\
& \text { Recall }_{i}=\frac{T P_{i}}{T P_{i}+F N_{i}} \\
& F_{1 i}=\frac{2 * \text { Precision }_{i} * \text { Recall }_{i}}{\text { Precision }_{i}+\text { Recall }_{i}} \\
& \text { Em que }\left\{\begin{array}{c}
T P_{i}=\# \text { de anotações positivas } \\
F P_{i}=\# \text { de anotações falso }- \text { positivas } \\
F N_{i}=\# \text { de anotações falso }- \text { negativas }
\end{array}\right.
\end{aligned}
$$

\section{3}

\section{Baselines}

Para medir o impacto do nosso sistema descrito no Capítulo 5 que usa word embeddings e character embeddings, nós criamos dois sistemas de baseline. Eles são usados como ponto de referência para comparar nossos resultados. Os resultados dos sistemas baseline podem informar se o modelo principal realmente agrega valor na classificação morfossintática.

O primeiro sistema baseline (Perceptron-features-manuais) usa um Perceptron multicamadas com features não automáticas.

Na Figura 6.8 temos a estrutura do Perceptron multicamadas utilizado como baseline. Usamos uma camada densa totalmente conectada na camada de entrada, camadas com a função de ativação RELU e camadas de dropout. Na camada de saída, usamos uma softmax como função de ativação. Nós usamos como features: a presença de letras maiúsculas, sufixos e prefixo das 


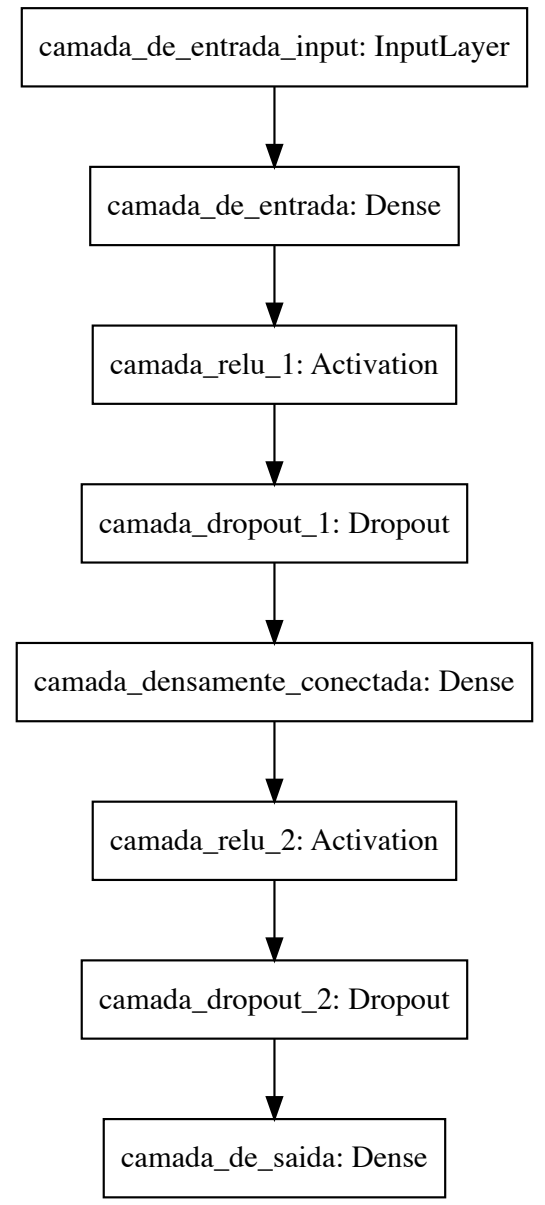

Figura 6.8: Arquitetura do baseline Perceptron-features-manuais

palavras e ocorrência das palavras (três) anteriores e posteriores. Nesse modelo não usamos qualquer tipo de informação de word embeddings ou character embeddings.

O segundo sistema baseline (BLSTM-WE) é semelhante ao nosso modelo principal, porém sem a camada de BLSTM para character embeddings, somente vetores de word embeddings. Na Figura 6.9 temos a arquitetura do segundo baseline. Também usamos uma camada de BLSTM e uma função de ativação softmax.

\section{4}

\section{Ajuste de Hiperparâmetro}

Redes Neurais contam com vários hiperparâmetros, esses valores controlam diretamente o processo de treinamento. O tamanho da camada escondida, o valor de dropout, e entre outras variáveis das redes neurais impactaram no desempenho dos modelos. Nessa seção procuramos por hiperparâmetros 


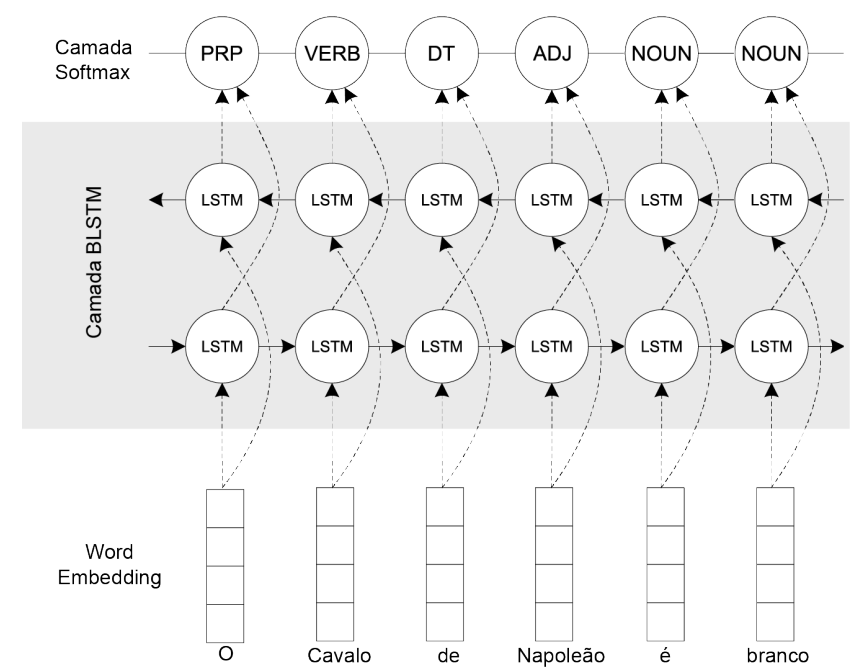

Figura 6.9: Arquitetura de baseline BLSTM-WE.

que maximizem a acurácia dos dois modelos baselines (Perceptron-featuresmanuais e BLSTM-WE) e do nosso modelo principal (BLSTM-WE-CE), apresentado no Capítulo 5. Usamos o conjunto de validação para procurar por hiperparâmetros e escolhemos o corpus Mac-Morpho v1 para esse processo.

Para o baseline Perceptron-features-manuais, queremos testar: Qual o tamanho de camada escondida produz melhores resultados? Qual valor de dropout produz melhores resultados? Os resultados dos experimentos encontram-se na Tabela 6.6. Primeiro procuramos pelo tamanho da camada escondida, o tamanho 400 obteve o melhor valor em acurácia no conjunto de validação. Depois procuramos pela a probabilidade de dropout, 0.4 produziu o melhor resultado. Um alto valor de dropout faz com que o modelo "esqueça" muitos neurônios e não aprenda algumas features importantes.

Tabela 6.6: Resultados dos experimentos do modelo Perceptron-featuresmanuais avaliado no conjunto de desenvolvimento do Mac-Morpho v1.

\begin{tabular}{lll}
\hline Camada Escondida & Dropout & Acurácia (dev.) \\
\hline 50 & 0.1 & $96.75 \%$ \\
100 & 0.1 & $96.77 \%$ \\
200 & 0.1 & $96.79 \%$ \\
400 & 0.1 & $\mathbf{9 6 . 8 4 \%}$ \\
600 & 0.1 & $96.78 \%$ \\
\hline 400 & 0.2 & $96.94 \%$ \\
400 & 0.3 & $97.00 \%$ \\
400 & 0.4 & $\mathbf{9 7 . 0 2} \%$ \\
400 & 0.5 & $96.96 \%$ \\
400 & 0.6 & $96.96 \%$ \\
400 & 0.7 & $96.40 \%$ \\
400 & 0.8 & $95.79 \%$ \\
\hline
\end{tabular}


Para o baseline BLSTM-WE, queremos testar: Qual o tamanho de camada escondida produz melhores resultados? Qual valor de dropout produz melhores resultados? Qual o melhor tamanho para o vetor de word embedding?

Tabela 6.7: Resultados dos experimentos do modelo BLSTM-WE avaliado no conjunto de desenvolvimento do Mac-Morpho v1.

\begin{tabular}{lllll}
\hline Camada Escondida & Dropout & Dimensão & W. embedding & Acurácia (dev.) \\
\hline 10 & 0.1 & 50 & Word2Vec & $95.65 \%$ \\
50 & 0.1 & 50 & Word2Vec & $96.75 \%$ \\
100 & 0.1 & 50 & Word2Vec & $96.92 \%$ \\
200 & 0.1 & 50 & Word2Vec & $96.91 \%$ \\
300 & 0.1 & 50 & Word2Vec & $\mathbf{9 7 . 0 1 \%}$ \\
400 & 0.1 & 50 & Word2Vec & $96.87 \%$ \\
500 & 0.1 & 50 & Word2Vec & $\mathbf{9 7 . 0 1 \%}$ \\
1000 & 0.1 & 50 & Word2Vec & $96.91 \%$ \\
1500 & 0.1 & 50 & Word2Vec & $96.85 \%$ \\
2000 & 0.1 & 50 & Word2Vec & $\mathbf{9 7 . 0 1} \%$ \\
\hline 500 & 0.2 & 50 & Word2Vec & $96.93 \%$ \\
500 & 0.3 & 50 & Word2Vec & $96.87 \%$ \\
500 & 0.4 & 50 & Word2Vec & $97.04 \%$ \\
500 & 0.5 & 50 & Word2Vec & $\mathbf{9 7 . 0 7} \%$ \\
500 & 0.6 & 50 & Word2Vec & $97.05 \%$ \\
500 & 0.7 & 50 & Word2Vec & $\mathbf{9 7 . 0 7 \%}$ \\
500 & 0.8 & 50 & Word2Vec & $97.02 \%$ \\
\hline 500 & 0.5 & 100 & Word2Vec & $\mathbf{9 7 . 1 5 \%}$ \\
500 & 0.5 & 300 & Word2Vec & $\mathbf{9 7 . 1 5} \%$ \\
\hline
\end{tabular}

Na Tabela 6.7, temos os resultados dos experimentos realizados usando baseline BLSTM-WE. Testamos o impacto do tamanho da camada escondida de BLSTM, esse hiperparâmetro tem um impacto limitado dado que a partir do tamanho 100, os resultados em acurácia não variam muito. Escolhemos o valor 500 com hiperparâmetro, esse valor apresenta o melhor resultado em acurácia no conjunto de validação e é um meio termo entre os outros valores que apresentaram também os melhores resultados.

Quando a probabilidade de dropout, 0.5 e 0.7 apresentaram os melhores resultados. Escolhemos 0.5 para o nosso sistema. O hiperparâmetro com maior impacto no resultado, foi o tamanho dos vetores de word embedding. O tamanho 100 e 300, produziram os mesmos resultados, e melhoram em relação ao tamanho 50 usado nas outras configurações. Escolhemos o tamanho 100 para o nosso sistema, ele produz o mesmo resultado que o tamanho 300 e traz menor complexidade de tamanho para o modelo.

$\mathrm{Na}$ Tabela 6.8 temos os resultados dos experimentos realizados para encontrar os hiperparâmetros para nossa arquitetura principal BLSTM-WECE. Procuramos pelo tamanho da camada escondida do BLSTM principal, o valor de dropout, tamanho da camada escondida do BLSTM que gera o character embedding, e o tamanho dos vetores de representação de word 
Tabela 6.8: Resultados dos experimentos do modelo BLSTM-WE-CE avaliado no conjunto de desenvolvimento do Mac-Morpho v1.

\begin{tabular}{llllll}
\hline Cam. E. & Dropout & Cam. E. Char. & Dimesão & W. embedding & Acurácia (val.) \\
\hline 10 & 0.1 & 10 & 50 & Word2Vec & $95.93 \%$ \\
50 & 0.1 & 10 & 50 & Word2Vec & $97.38 \%$ \\
100 & 0.1 & 10 & 50 & Word2Vec & $97.56 \%$ \\
200 & 0.1 & 10 & 50 & Word2Vec & $\mathbf{9 7 . 7 3 \%}$ \\
300 & 0.1 & 10 & 50 & Word2Vec & $97.67 \%$ \\
400 & 0.1 & 10 & 50 & Word2Vec & $97.67 \%$ \\
500 & 0.1 & 10 & 50 & Word2Vec & $\mathbf{9 7 . 7 3 \%}$ \\
1000 & 0.1 & 10 & 50 & Word2Vec & $97.72 \%$ \\
1500 & 0.1 & 10 & 50 & Word2Vec & $97.73 \%$ \\
2000 & 0.1 & 10 & 50 & Word2Vec & $97.66 \%$ \\
\hline 500 & 0.2 & 10 & 50 & Word2Vec & $97.72 \%$ \\
500 & 0.3 & 10 & 50 & Word2Vec & $97.67 \%$ \\
500 & 0.4 & 10 & 50 & Word2Vec & $97.61 \%$ \\
500 & 0.5 & 10 & 50 & Word2Vec & $97.73 \%$ \\
500 & 0.6 & 10 & 50 & Word2Vec & $\mathbf{9 7 . 7 9 \%}$ \\
500 & 0.7 & 10 & 50 & Word2Vec & $97.72 \%$ \\
500 & 0.8 & 10 & Word2Vec & $97.54 \%$ \\
\hline 500 & 0.6 & 20 & Word2Vec & $97.67 \%$ \\
500 & 0.6 & 30 & Word2Vec & $97.75 \%$ \\
500 & 0.6 & 40 & Word2Vec & $97.69 \%$ \\
500 & 0.6 & 50 & Word2Vec & $\mathbf{9 7 . 7 9 \%}$ \\
500 & 0.6 & 100 & Word2Vec & $97.74 \%$ \\
\hline 500 & 0.6 & 50 & Word2Vec & $\mathbf{9 7 . 8 0 \%}$ \\
500 & 0.6 & 50 & Word2Vec & $97.74 \%$ \\
\hline
\end{tabular}

embedding. Primeiro testamos o tamanho da camada escondida do BLSTM, 200 e 500 apresentaram os melhores resultados em acurácia, e escolhemos o tamanho 500. Depois procuramos pelo o valor de dropout, a probabilidade 0.6 gerou o melhor resultado entre as outras probabilidades testadas.

Procuramos também pelo tamanho da camada escondida do BLSTM que gera o character embedding, testamos os tamanhos 10, 20, 30, 40, 50 e 100. O tamanho 50 gerou o melhor resultado em acurácia no conjunto de validação. E por último, analisamos qual o melhor tamanho para o vetor de word embedding, testamos os tamanhos 50, 100 e 300, o tamanho 100 produziu o melhor resultado em acurácia.

Na Tabela 6.9, temos o resumo dos hiperparâmetros escolhidos para as arquiteturas dos baselines e a arquitetura principal. Todos os experimentos realizados com essas arquiteturas usam essas configurações no processo de treino.

\section{5}

\section{Análise de word embedding}

Nossa arquitetura (BLSTM-WE-CE) usa representações de word embedding. Esses vetores são pré-treinados em grandes corpora textuais não su- 
Tabela 6.9: Hiperparâmetros selecionados para as arquiteturas baseline e arquitetura principal.

Perceptron-features-manuais

\begin{tabular}{ll}
\hline Hiperparâmetro & Valor \\
\hline Camada Escondida & 400 \\
Dropout & 0.4 \\
\hline
\end{tabular}

\section{BLSTM-WE}

\begin{tabular}{ll}
\hline Hiperparâmetro & Valor \\
\hline Camada Escondida (BLSTM) & 500 \\
Dropout (BLSTM) & 0.5 \\
Dimensão word embedding & 100 \\
\hline
\end{tabular}

\section{BLSTM-WE-CE}

\begin{tabular}{ll}
\hline Hiperparâmetro & Valor \\
\hline Camada Escondida (BLSTM) & 500 \\
Dropout (BLSTM) & 0.6 \\
Camada Escondida (char. embedding) & 50 \\
Dimensão word embedding & 100 \\
\hline
\end{tabular}

pervisionadas. Nesse trabalho, analisamos quais algoritmos de treino de word embedding geram os melhores resultado em acurácia na classificação morfossintática para a Língua Portuguesa. Analisamos os algoritmos Word2vec, Fasttext, Wang2vec and Glove.

Para nossos experimentos usamos as representações word embedding NILC-Embeddings, criadas e disponibilizadas pelo Núcleo Interinstitucional de Linguística Computacional (NILC) no trabalho (28). Esses vetores foram gerados a partir da junção de várias corporas em Português, de diferentes fontes e domínios, a fim de obter representações de gêneros variados. Foram usados 17 corpora diferentes que totalizam 1.395.926.282 tokens. O treinamento dos vetores ocorreu com os algoritmos Word2vec, FastText, Wang2vec e Glove e estão disponíveis no repositório online ${ }^{5}$.

Nós testamos o desempenho do nosso modelo nos quatro métodos diferentes (Word2vec, FastText, Wang2vec e Glove) e suas versões de CBOW e Skip-Gram. Usamos as métricas de acurácia. Na Tabela 6.10 apresentamos os resultados no conjunto de validação para os três corpora.

Todos os experimentos realizados apresentaram alta acurácia (mais que 97\%). As representações geradas pelo Wang2Vec obtiveram os melhores resultados em acurácia nos três corpora analisadas. O bom desempenho de Wang2vec pode ser explicado por seu foco na captura de informações sintáticas, que leva em conta a ordem em que as palavras aparecem (34), isso se

${ }^{5}$ http://nilc.icmc.usp.br/embeddings 
mostra bastante útil para POS tagging.

Para o corpus Mac-Morpho v1, os melhores resultados foram obtidos na configuração que usa o Wang2Vec CBOW, com $97.97 \%$ de acurácia no cojunto de validação. Para o corpus Mac-Morpho v2, os melhores resultados em acurácia também foram obtidos com o Wang2Vec CBOW (97.67\% de acurácia), com uma pequena margem de $0.02 \%$ de diferença para os resultados obtidos com o Wang2Vec Skip-Gram. No corpus Tycho Brahe, a configuração que usa o Wang2Vec Skip-Gram obteve os melhores resultados em acurácia de $97.30 \%$.

Concluímos que a mudança do tipo algoritmo de word embedding resulta em um impacto nos resultados em acurácia para a atividade de POS tagging. O modelo Wang2Vec apresentou os melhores resultados em acurácia para os três corpora, porém esses resultados não tiveram um grande distanciamento dos resultados em acurácia obtidos por outros modelos de word embedding.

Tabela 6.10: Desempenho do nosso modelo com diferentes word embeddings no conjunto validação.

\begin{tabular}{|c|c|c|c|}
\hline Corpus & Modelos c & e Embedding & Ac. $(\%)$ \\
\hline \multirow{7}{*}{ Mac-Morpho v1 } & \multirow{2}{*}{ Word2Vec } & CBOW & 97.84 \\
\hline & & Skip-Gram & 97.72 \\
\hline & \multirow{2}{*}{ FastText } & CBOW & 97.78 \\
\hline & & Skip-Gram & 97.80 \\
\hline & \multirow{2}{*}{ Wang2Vec } & CBOW & 97.97 \\
\hline & & Skip-Gram & 97.84 \\
\hline & Glove & & 97.70 \\
\hline \multirow{7}{*}{ Mac-Morpho v2 } & \multirow{2}{*}{ Word2Vec } & $\mathrm{CBOW}$ & 97.56 \\
\hline & & Skip-Gram & 97.59 \\
\hline & \multirow{2}{*}{ FastText } & CBOW & 97.44 \\
\hline & & Skip-Gram & 97.55 \\
\hline & \multirow{2}{*}{ Wang2Vec } & CBOW & 97.67 \\
\hline & & Skip-Gram & 97.65 \\
\hline & Glove & & 97.53 \\
\hline \multirow{7}{*}{ Tycho Brahe } & \multirow{2}{*}{ Word2Vec } & CBOW & 96.93 \\
\hline & & Skip-Gram & 97.01 \\
\hline & \multirow{2}{*}{ FastText } & CBOW & 96.94 \\
\hline & & Skip-Gram & 97.07 \\
\hline & \multirow{2}{*}{ Wang2Vec } & CBOW & 97.17 \\
\hline & & Skip-Gram & 97.30 \\
\hline & Glove & & 97.11 \\
\hline
\end{tabular}




\subsection{1}

\section{Comparação com baselines}

Para medir o impacto do uso de word embeddings e character embeddings, nós comparamos a performance do nosso modelo principal com os dois sistemas baseline descritos na seção 6.3. Os hiperparâmetros usados nos experimentos são os mesmos apresentados na seção 6.4. O modelo de word embeddings usado é o Wang2Vec, tanto para o baseline BLSTM-WE quanto para o modelo principal BLSTM-WE-CE.

Na Tabela 6.11 temos a acurácia e acurácia FV dos três sistemas nos três corpora avaliados. O baseline BLSTM-WE alcança melhores resultados que Perceptron-features-manuais em Mac-Morpho v1 e Mac-Morpho v2. Porém, no corpus Tycho Brahe, o modelo Perceptron-features-manuais performa melhor. Isso pode se explicado pelo fato que corpus Tycho Brahe é formado por textos do Português Históricos e algumas palavras que estão presentes no corpus, não estão presentes no modelo pré-treinado de word embeddings. Isso faz com que o corpus Tycho Brahe não se beneficie com os outros corpora das informações de word embeddings.

Nosso sistema principal BLSTM-WE-CE supera os dois sistemas de baseline nos três corpora avaliados. Esses resultados suportam os resultados já demonstrados por (44) e (25), de que informações de character embeddings são muito importantes para a etiquetagem de sequência em atividades como POS tagging.

Comparando nosso sistema principal BLSTM-WE-CE e o primeiro sistema baseline Perceptron-features-manuais, o corpus Mac-Morpho v1 teve um aumento de 1.29 pontos de acurácia e de 7 pontos de acurácia FV. O corpus Mac-Morpho v2 teve um aumento de 1.08 pontos de acurácia e de 4.57 pontos de acurácia FV. Já o corpus Tycho Brahe obteve um aumento de 1.14 em pontos de acurácia e 10.84 em pontos de acurácia FV.

O sistema BLSTM-WE-CE tem um maior impacto no corpus Tycho Brahe em acurácia FV. Isso se deve ao fato de que o corpus Tycho Brahe possui muitas classes que descrevem informações morfológicas (gênero, número e tempo verbal) das palavras, justamente o tipo de informação abundante nos de vetores de character embeddings.

Os resultados empíricos do nosso sistema proposto (BLSTM-WECE), mostram que vetores de character embeddings gerados por uma camada BLSTM contribuem bastante para a classificação morfossintática da Língua Portuguesa. Observamos um maior ganho em acurácia FV quando usamos character embeddings nos três corpora. Esses resultados indicam que o uso de tais representações melhora o desempenho do modelo em palavras que não 
fazem parte do vocabulário de treino ou do modelo de word embeddings.

Tabela 6.11: Desempenho dos nossos modelos baseline no conjunto de teste

\begin{tabular}{llll}
\hline Corpus & Sistema & Ac. (\%) & Ac. FV (\%) \\
\hline \multirow{3}{*}{ Mac-Morpho v1 } & Perceptron-features-manuais & 96.59 & 88.59 \\
& BLSTM-WE & 96.74 & 89.76 \\
& BLSTM-WE-CE & $\mathbf{9 7 . 8 8}$ & $\mathbf{9 5 . 5 9}$ \\
\hline \multirow{3}{*}{ Mac-Morpho v2 } & Perceptron-features-manuais & 96.56 & 90.54 \\
& BLSTM-WE & 96.60 & 90.80 \\
& BLSTM-WE-CE & $\mathbf{9 7 . 6 4}$ & $\mathbf{9 5 . 1 1}$ \\
\hline \multirow{3}{*}{ Tycho Brahe } & Perceptron-features-manuais & 96.27 & 76.86 \\
& BLSTM-WE & 95.90 & 75.25 \\
& BLSTM-WE-CE & $\mathbf{9 7 . 4 1}$ & $\mathbf{8 7 . 7 0}$ \\
\hline
\end{tabular}

Na Tabela 6.12 mostrados o tempo computacional gasto para treinar o modelo BLSTM-WE-CE nos três diferentes corpora, os experimentos foram realizados usando um GPU GeForce GTX TITAN X. Para treinar os corpora Mac-Morpho v1 e Mac-Morpho v2 gastamos aproximadamente 45 minutos e treinar o corpus Tycho Brahe gastamos aproximadamente 31 minutos.

Tabela 6.12: Tempo de treino aproximado nos três diferentes corpora

\begin{tabular}{rr}
\hline Corpus & Tempo de treino $(\approx)$ \\
\hline Mac-Morpho v1 & 45 minutos \\
Mac-Morpho v2 & 45 minutos \\
Tycho Brahe & 31 minutos \\
\hline
\end{tabular}



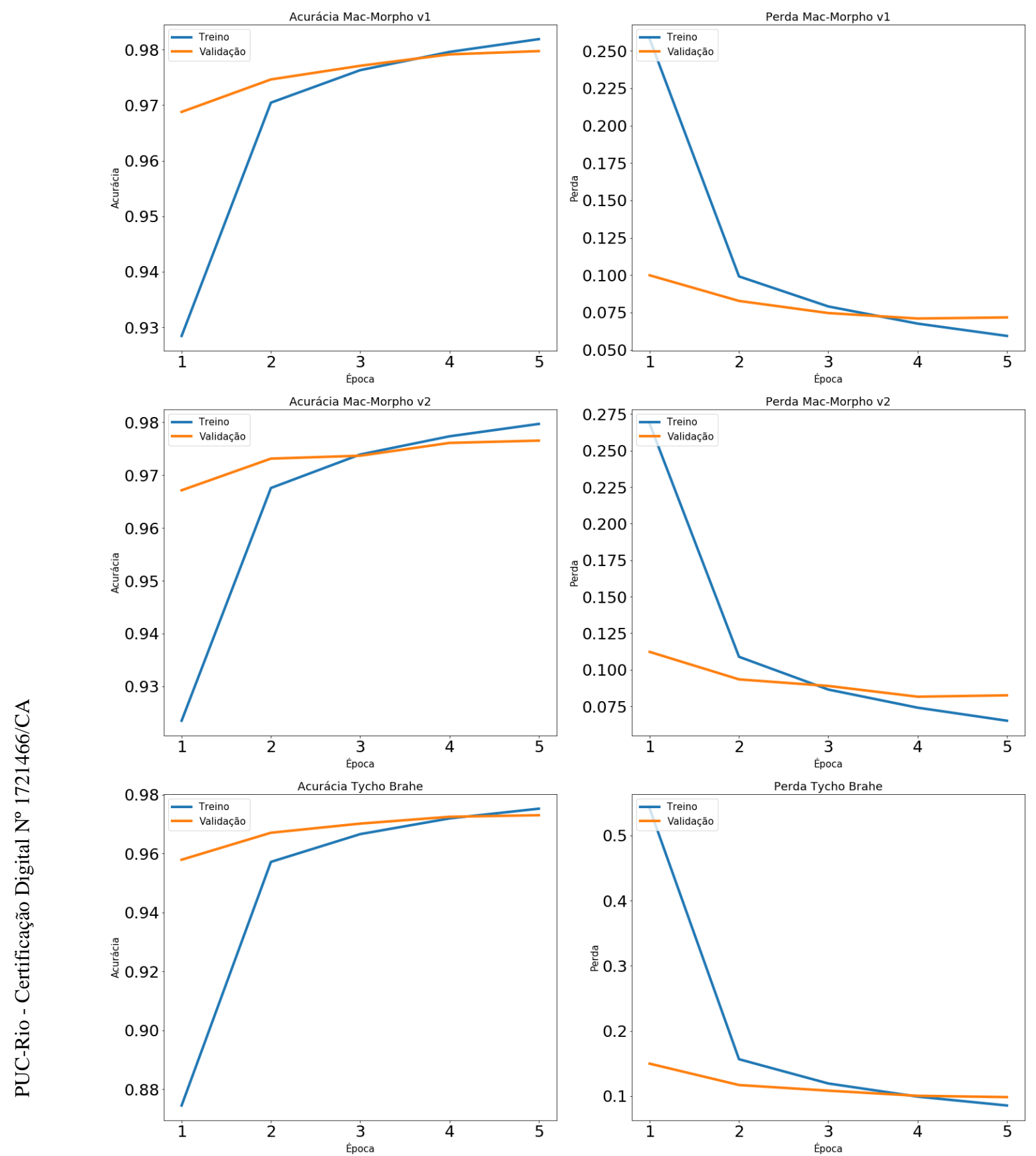

Figura 6.10: Treino do modelo BLSTM-WE-CE nas corpora Mac-Morpho v1, Mac-Morpho v2 e Tycho Brahe.

Na Figura 6.10, mostramos acurácia e perda do conjunto de treino e validação durante as épocas do treino do nosso modelo BLSTM-WE-CE. A perda foi calcula usando a função sparse categorical crossentropy. Para os três corpora, o valor de acurácia do conjunto de treino, ultrapassou o valor de acurácia do conjunto validação depois da terceira época. Isso pode indicar que depois da época três o modelo começa a se especializar no conjunto de treino, podendo perder generalidade.

Podemos observar o mesmo comportamento olhando a perda dos corpora 
durante o treino. Por volta da terceira época o valor de perda do conjunto de validação ultrapassou o conjunto de treino. Podendo indicar também o começo de uma especialização no conjunto de treino. Por isso, treinamos com apenas cinco épocas.

\section{5 .2}

\section{Comparação com outros trabalhos}

Seguindo a metodologia adotada por Peters et al. (69) e Chiu e Nichols (70), nós treinamos nosso modelo principal BLSTM-WE-CE dez vezes com diferentes sementes aleatória e reportamos média e desvio padrão de acurácia e acurácia FV. Isso é importante para estimar a variância dos resultados do nosso modelo, já que durante o treino existe algum fator de aleatoriedade associado, com por exemplo, as camadas de dropout.

Tabela 6.13: Resultados no conjunto de teste para dez treinos diferentes com sementes aleatórias.

\begin{tabular}{cll|ll|ll}
\hline \multicolumn{2}{l}{\begin{tabular}{l}
\multicolumn{2}{l}{ Mac-Morpho v1 } \\
Ac.
\end{tabular}} & \multicolumn{2}{l}{ Ac. FV } & \multicolumn{2}{l}{ Ac. Ac. } & \multicolumn{2}{l}{ Ac. FV } & \multicolumn{2}{l}{ Ac. } & \multicolumn{1}{l}{ Ac. FV } \\
\hline 97.84 & 95.35 & 97.64 & 95.11 & 97.37 & 87.53 \\
97.86 & 95.47 & 97.67 & 95.28 & 97.34 & 88.13 \\
97.82 & 95.74 & 97.63 & 95.28 & 97.35 & 87.28 \\
97.82 & 95.54 & 97.66 & 94.93 & 97.41 & 87.70 \\
& 97.85 & 95.50 & 97.65 & 95.51 & 97.38 & 87.93 \\
97.82 & 95.48 & 97.67 & 94.97 & 97.36 & 86.95 \\
& 97.81 & 95.51 & 97.63 & 95.46 & 97.30 & 86.83 \\
& 97.83 & 95.41 & 97.68 & 95.11 & 97.33 & 87.53 \\
& 97.84 & 95.27 & 97.68 & 95.42 & 97.33 & 87.14 \\
& 97.88 & 95.59 & 97.65 & 95.28 & 97.37 & 86.99 \\
$\bar{x}$ & 97.83 & 95.48 & 97.65 & 95.23 & 97.35 & 87.40 \\
$\sigma$ & 0.021 & 0.129 & 0.018 & 0.2 & 0.030 & 0.436 \\
$\min$ & 97.81 & 95.27 & 97.63 & 94.93 & 97.30 & 86.83 \\
$\max$ & 97.88 & 95.74 & 97.68 & 95.51 & 97.41 & 88.13 \\
\hline
\end{tabular}

Na Tabela 6.13, reportamos os resultados obtidos no conjunto de teste de dez treinos diferentes como sementes aleatórias. Para as métricas de avaliação acurácia e acurácia FV, calculamos média aritmética $(\bar{x})$, desvio padrão $(\sigma)$, o valor máximo $(\max )$ e o valor mínimo (min).

Na Figura 6.11, apresentamos a visualização das distribuições descritas na Tabela 6.13 com o gráfico boxplot. Podemos observar no boxplot do corpus Mac-Morpho v1, que os valores de acurácia reportados nos experimentos apresentam menor variabilidade que os valor de acurácia FV. Para o corpus Mac-Morpho v1, temos um valor médio de acurácia de $97.83 \%$ com desvio 
padrão de apenas $0.021 \%$ e um valor médio de acurácia FV de $95.48 \%$ com um desvio padrão de $0.129 \%$.

O corpus Mac-Morpho v2 apresenta uma avaliação com características semelhantes ao Mac-Morpho v1, como é possível notar na Figura 6.11. MacMorpho v2 também apresenta um espalhamento menor dos resultados de acurácia se comparados aos resultados de acurácia FV. Essa diferença talvez possa ser explicada pelo fato do conjunto de palavras fora do vocabulário ser bem menor que o conjunto todas as palavras. E conjuntos pequenos podem ser mais sensíveis a variações randômicas que possam acontecer no modelo no momento do treino. O Mac-Morpho v2 obteve na avaliação, acurácia média de $97.65 \%$ com um valor de desvio padrão de $0.018 \%$ e acurácia FV média de $95.23 \%$ com um desvio padrão de $0.2 \%$.

Para o corpus Tycho Brahe reportamos $97.35 \%$ de acurácia média com um desvio padrão de $0.030 \%$ e $87.40 \%$ de acurácia FV com desvio padrão de $0.436 \%$. Nesse corpus também observamos um menor espalhamento do valor de acurácia se comparado ao espalhamento do valor de acurácia FV.

Nós comparamos os resultados do modelo BLSTM-WE-CE com dois sistemas de melhores performance reportadas para a Língua Portuguesa. O primeiro trabalho de Dos Santos e Zadrozny (44) que usaram, representações word embeddings e character embeddings, combinados ao algoritmo de Viterbi para fazer a predição das etiquetas. E o trabalho de Fonseca et al. (45), que usa uma rede perceptron multicamadas combinada a word embeddings e algumas features manuais. Usamos a mesma partição treino/teste/validação que foram usadas nesses trabalhos.

Tabela 6.14: Comparação com outros POS taggers em Português

\begin{tabular}{llll}
\hline Corpus & Sistema & Ac. (\%) $\pm \sigma$ & Ac. FV (\%) \\
\hline \multirow{3}{*}{ Mac-Morpho v1 } & Este Trabalho & $\mathbf{9 7 . 8 3} \pm \mathbf{0 . 0 2 1}$ & $\mathbf{9 5 . 4 8} \pm \mathbf{0 . 1 2 9}$ \\
& Fonseca et al. 2015 (45) & 97.57 & 93.38 \\
& Santos e Zadrozny 2014 (44) & 97.47 & 92.49 \\
\hline \multirow{3}{*}{ Mac-Morpho v2 } & Este Trabalho & $\mathbf{9 7 . 6 5} \pm \mathbf{0 . 0 1 8}$ & $\mathbf{9 5 . 2 3} \pm \mathbf{0 . 2}$ \\
& Fonseca et al. 2015 (45) & 97.48 & 94.34 \\
& Santos e Zadrozny 2014 (44) & 97.31 & 93.43 \\
\hline \multirow{3}{*}{ Tycho Brahe } & Este Trabalho & $\mathbf{9 7 . 3 5} \pm \mathbf{0 . 0 3 0}$ & $\mathbf{8 7 . 4 0} \pm \mathbf{0 . 4 3 6}$ \\
& Fonseca et al. 2015 (45) & 96.91 & 84.14 \\
& Santos e Zadrozny 2014 (44) & 97.17 & 86.58 \\
\hline
\end{tabular}

Na Tabela 6.14, nós comparamos acurácia média e acurácia FV média dos resultados obtidos com os valores reportados nos trabalhos anteriores para a Língua Portuguesa. No corpus Mac-Morpho v1 aumentamos 0.26 pontos de acurácia em média, e 2.02 de acurácia FV em média, se compararmos com o resultado reportado por Fonseca et al. (45). 

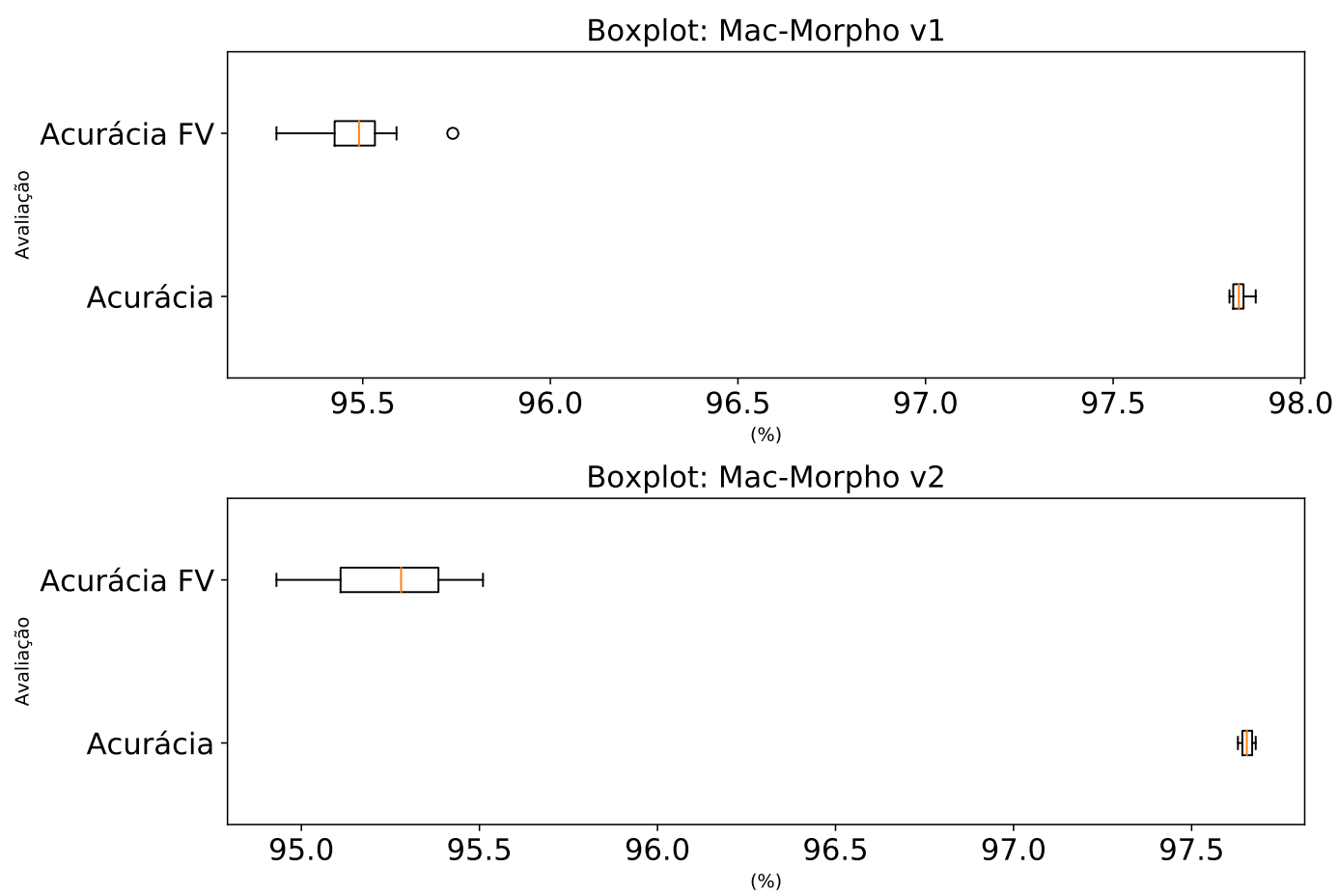

Boxplot: Tycho Brahe

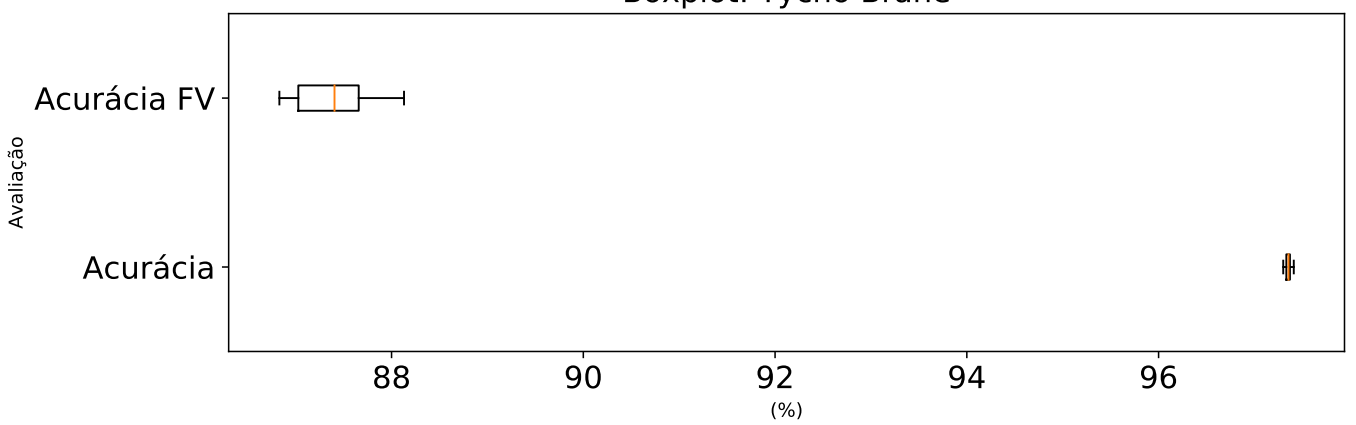

Figura 6.11: Boxplot dos resultados de 10 diferentes treinos com o modelo BLSTM-WE-CE nos corpora Mac-Morpho v1, Mac-Morpho v2 e Tycho Brahe.

No Mac-Morpho v2 aumentamos 0.17 pontos de acurácia em média, e 1.8 pontos de acurácia FV em média, se compararmos com o resultado reportado por Fonseca et al (45). Já no corpus Tycho Brahe aumentamos 0.18 pontos de acurácia em média, e 0.82 pontos de acurácia FV em média, se compararmos com o resultado reportado por Santos e Zadrozny (44). Os resultados definem um novo estado da arte nos três corpora usados, demonstrando assim a efetividade do uso de BLSTM combinado a word embeddings e character embeddings para POS tagging em Português.

\section{6}

\section{Análise por etiquetas}

Nós usamos $F_{1}$-score, precision e recall para avaliar os 10 experimentos realizados com o modelo $B L S T M-W E-C E$. Também realizamos uma análise do desempenho médio do modelo por etiquetas. Na Tabela 6.15 mostramos a 
média de avaliação das etiquetas para os três corpora.

Tabela 6.15: Valor médio de $F_{1}$, Precision e Recall dos experimentos realizados com o BLSTM-WE-CE no conjunto de teste

\begin{tabular}{rrrr}
\hline Corpus & $\boldsymbol{F}_{\mathbf{1}} \pm \sigma$ & Precision $\pm \sigma$ & Recall $\pm \sigma$ \\
\hline Mac-Morpho v1 & $0.948 \pm 0.094$ & $0.953 \pm 0.075$ & $0.945 \pm 0.108$ \\
Mac-Morpho v2 & $0.902 \pm 0.011$ & $0.908 \pm 0.022$ & $0.899 \pm 0.014$ \\
Tycho Brahe & $0.740 \pm 0.045$ & $0.753 \pm 0.054$ & $0.743 \pm 0.049$ \\
\hline
\end{tabular}

O corpus Mac-Morpho v1 obteve a melhor avaliação média $\left(F_{1}=0.948\right.$, precision $=0.953$, recall $=0.945)$. Esse corpus possui muitas etiquetas de pontuação, tais etiquetas são fáceis de classificar e quase sempre o modelo acerta $100 \%$ dos exemplos dessas classes, isso faz com que a média final do corpus seja influenciada por esses valores. Na Tabela 6.16 apresentamos os valores de $F_{1}$, precision e recall médio para cada etiqueta do corpus Mac-Morpho v1. Nessa tabela podemos ver que as etiquetas de pontuação quase sempre obtêm 100\% de $F_{1}$.

Na Figura 6.12, para melhor comparação entre valores, mostramos o $F_{1}$ médio das classes presentes no conjunto de treino, e que não são classes de pontuação do corpus Mac-Morpho v1. A maioria das etiquetas obtiveram um $F_{1}$ de mais de $95 \%$. As piores avaliações foram das etiquetas ADV-KS (Advérbio conectivo de subordinação) com $52 \%$ de $F_{1}$ e IN (Interjeição) com $66.1 \%$ de $F_{1}$. Essas duas etiquetas, estão entre as classes de mais baixa representação no corpus Mac-Morpho v1. A etiqueta ADV-KS representa apenas $0.034 \%$ do total de tokens, já a etiqueta IN representa apenas $0.032 \%$ do total. Devido às baixas distribuições, o modelo não conseguiu aprender a classificar essas etiquetas tão bem quanto as outras.

O corpus Mac-Morpho v2 obteve a segunda melhor avaliação média $\left(F_{1}=0.902\right.$, precision $=0.908$ e recall $\left.=0.899\right)$ no conjunto de treino. Na Tabela 6.17 apresentamos os valores de $F_{1}$, precision e recall médio para cada uma das 30 etiquetas do corpus Mac-Morpho v2. E na Figura 6.13, para melhor comparação entre os resultados, apresentamos o $F_{1}$ médio das classes presentes no Mac-Morpho v2.

Podemos ver na Figura 6.13 que a etiqueta com a pior avaliação no Mac-Morpho v2 é PREP+PRO-KS (Preposição + subordinação do pronome relativo conectivo). Essa etiqueta obteve apenas $9.9 \%$ de $F_{1}$. O modelo não aprendeu a reconhecer bem a classe PREP+PRO-KS, provavelmente devido a baixa representação no corpus. Essa etiqueta tem a pior representatividade, com apenas 32 exemplos em todo o corpus.

Ainda na Figura 6.13 vemos que a etiqueta ADV-KS obteve $64.4 \%$ de $F_{1}$, essa classe representa apenas $0.03 \%$ dos tokens presentes no Mac- 
Tabela 6.16: Valor médio de $F_{1}$, Precision e Recall por etiqueta no conjunto de treino do corpus Mac-Morpho v1

\begin{tabular}{|c|c|c|c|}
\hline Classe & $\boldsymbol{F}_{\mathbf{1}} \pm \sigma$ & $\boldsymbol{P r e c i s i o n} \pm \sigma$ & $\operatorname{Recall}_{ \pm \sigma}$ \\
\hline ! & $1.000 \pm 0.000$ & $1.000 \pm 0.000$ & $1.000 \pm 0.000$ \\
\hline " & $1.000 \pm 0.000$ & $1.000 \pm 0.000$ & $1.000 \pm 0.000$ \\
\hline , & $1.000 \pm 0.000$ & $1.000 \pm 0.000$ & $1.000 \pm 0.000$ \\
\hline( & $1.000 \pm 0.000$ & $1.000 \pm 0.000$ & $1.000 \pm 0.000$ \\
\hline ) & $1.000 \pm 0.000$ & $1.000 \pm 0.000$ & $1.000 \pm 0.000$ \\
\hline , & $1.000 \pm 0.000$ & $1.000 \pm 0.000$ & $1.000 \pm 0.000$ \\
\hline- & $0.981 \pm 0.003$ & $0.971 \pm 0.006$ & $0.990 \pm 0.000$ \\
\hline . & $1.000 \pm 0.000$ & $1.000 \pm 0.000$ & $1.000 \pm 0.000$ \\
\hline$\ldots$ & $1.000 \pm 0.000$ & $1.000 \pm 0.000$ & $1.000 \pm 0.000$ \\
\hline / & $1.000 \pm 0.000$ & $1.000 \pm 0.000$ & $1.000 \pm 0.000$ \\
\hline : & $1.000 \pm 0.000$ & $1.000 \pm 0.000$ & $1.000 \pm 0.000$ \\
\hline ; & $1.000 \pm 0.000$ & $1.000 \pm 0.000$ & $1.000 \pm 0.000$ \\
\hline$?$ & $1.000 \pm 0.000$ & $1.000 \pm 0.000$ & $1.000 \pm 0.000$ \\
\hline & $1.000 \pm 0.000$ & $1.000 \pm 0.000$ & $1.000 \pm 0.000$ \\
\hline ADJ & $0.950 \pm 0.000$ & $0.949 \pm 0.006$ & $0.957 \pm 0.005$ \\
\hline ADV & $0.940 \pm 0.000$ & $0.941 \pm 0.006$ & $0.934 \pm 0.005$ \\
\hline ADV-KS & $0.585 \pm 0.024$ & $0.764 \pm 0.063$ & $0.481 \pm 0.048$ \\
\hline ADV-KS-REL & $0.877 \pm 0.016$ & $0.882 \pm 0.042$ & $0.877 \pm 0.048$ \\
\hline ART & $0.990 \pm 0.000$ & $0.990 \pm 0.000$ & $0.993 \pm 0.005$ \\
\hline CUR & $1.000 \pm 0.000$ & $0.991 \pm 0.003$ & $1.000 \pm 0.000$ \\
\hline IN & $0.661 \pm 0.039$ & $0.666 \pm 0.071$ & $0.665 \pm 0.053$ \\
\hline $\mathrm{KC}$ & $0.980 \pm 0.000$ & $0.987 \pm 0.005$ & $0.978 \pm 0.004$ \\
\hline $\mathrm{KS}$ & $0.915 \pm 0.005$ & $0.916 \pm 0.013$ & $0.911 \pm 0.012$ \\
\hline $\mathrm{N}$ & $0.978 \pm 0.004$ & $0.978 \pm 0.004$ & $0.974 \pm 0.005$ \\
\hline NPROP & $0.968 \pm 0.004$ & $0.970 \pm 0.000$ & $0.960 \pm 0.000$ \\
\hline NUM & $0.970 \pm 0.000$ & $0.967 \pm 0.007$ & $0.967 \pm 0.007$ \\
\hline PCP & $0.970 \pm 0.000$ & $0.959 \pm 0.006$ & $0.982 \pm 0.006$ \\
\hline PDEN & $0.910 \pm 0.005$ & $0.917 \pm 0.016$ & $0.901 \pm 0.017$ \\
\hline PREP & $0.990 \pm 0.000$ & $0.990 \pm 0.000$ & $0.990 \pm 0.000$ \\
\hline PRO-KS & $0.749 \pm 0.014$ & $0.787 \pm 0.034$ & $0.718 \pm 0.049$ \\
\hline PRO-KS-REL & $0.940 \pm 0.000$ & $0.927 \pm 0.013$ & $0.953 \pm 0.012$ \\
\hline PROADJ & $0.980 \pm 0.000$ & $0.977 \pm 0.005$ & $0.980 \pm 0.000$ \\
\hline PROPESS & $0.980 \pm 0.000$ & $0.980 \pm 0.000$ & $0.985 \pm 0.005$ \\
\hline PROSUB & $0.884 \pm 0.005$ & $0.869 \pm 0.019$ & $0.901 \pm 0.014$ \\
\hline V & $0.990 \pm 0.000$ & $0.986 \pm 0.005$ & $0.990 \pm 0.000$ \\
\hline VAUX & $0.960 \pm 0.000$ & $0.967 \pm 0.007$ & $0.955 \pm 0.005$ \\
\hline Média \pm & $0.948 \pm 0.094$ & $0.953 \pm 0.075$ & $0.945 \pm 0.108$ \\
\hline
\end{tabular}




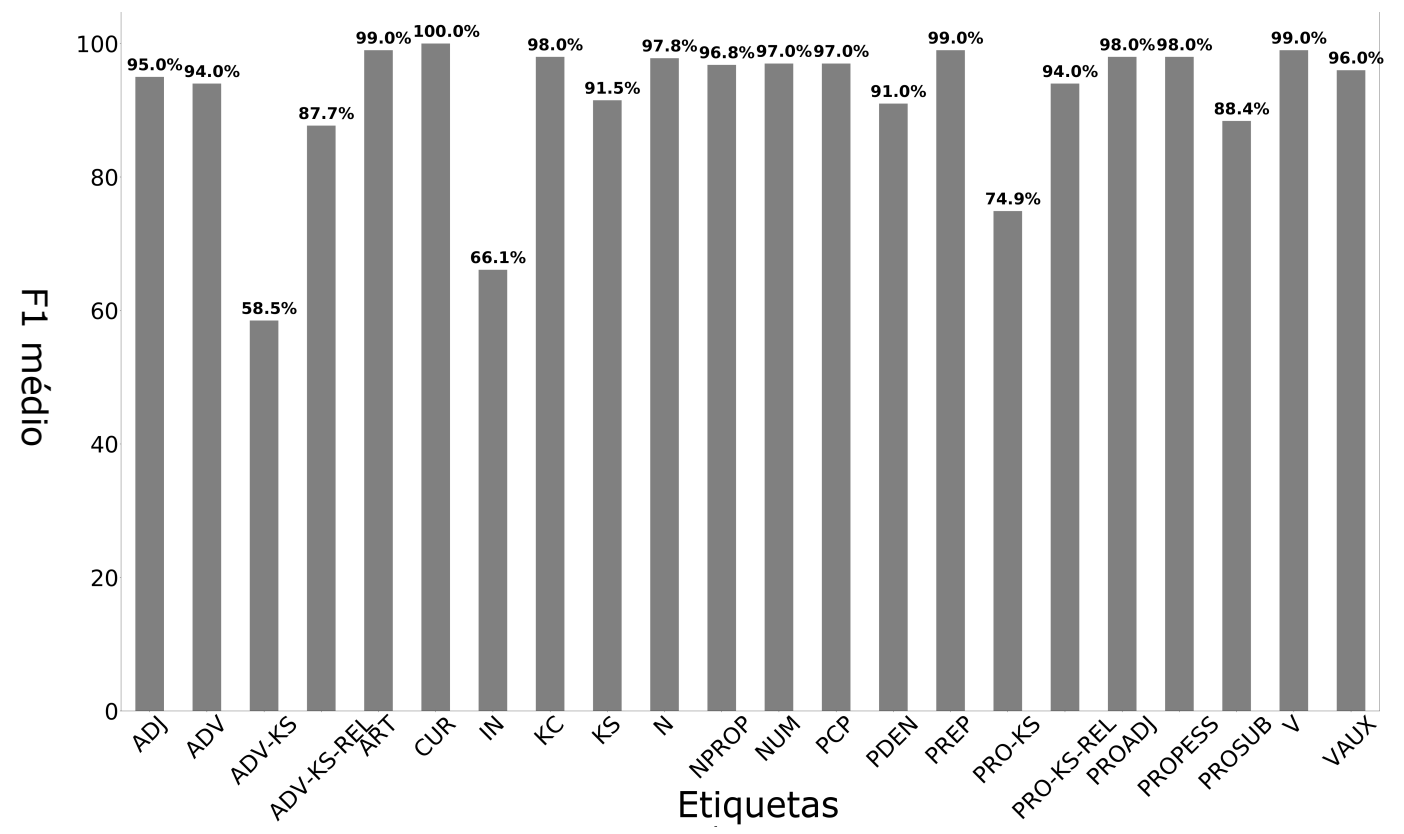

Figura 6.12: Valor médio de $F_{1}$ dos 10 experimentos realizados com o modelo BLSTM-WE-CE no corpus Mac-Morpho v1.

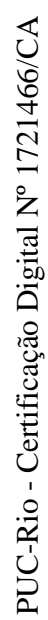

Morpho v2. A etiqueta IN obteve $70.0 \%$ de $F_{1}$, essa etiqueta também tem uma baixa representação no corpus de apenas 0.03\%. Essas classes obtiveram as piores avaliações, o modelo não aprendeu a reconhecer bem essas classes provavelmente devido a baixa representação. Para as outras classes do MacMorpho v2 temos um valor médio de $F_{1}$ entorno de $90 \%$.

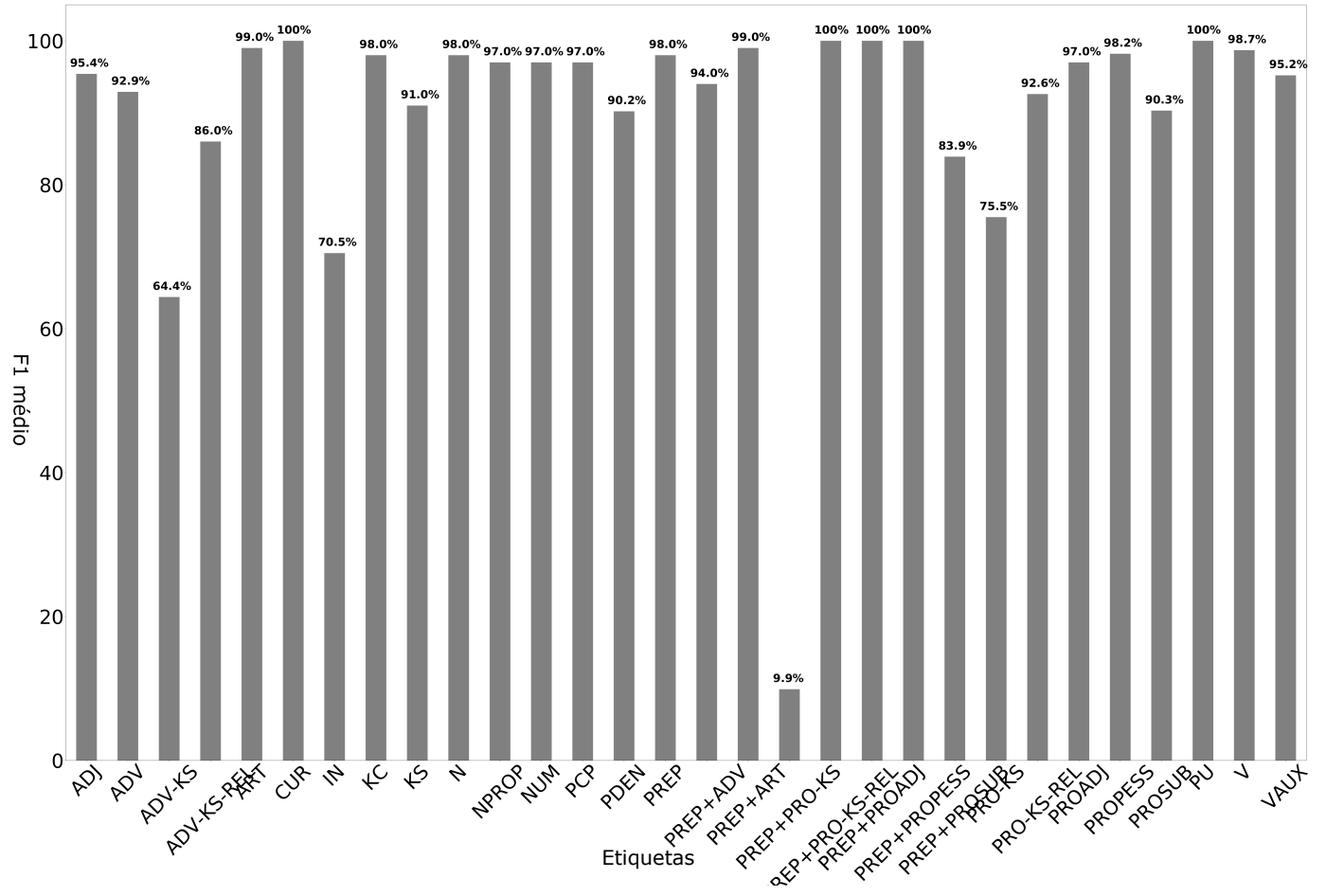

Figura 6.13: Valor médio de $F_{1}$ dos 10 experimentos realizados com o modelo BLSTM-WE-CE no corpus Mac-Morpho v2. 
Tabela 6.17: Valor médio de $F_{1}$, Precision e Recall por etiqueta no conjunto de treino do corpus Mac-Morpho v2

\begin{tabular}{rrrr}
\hline Classe & $\boldsymbol{F}_{\mathbf{1}} \pm \sigma$ & Precision $\pm \sigma$ & $\boldsymbol{R e c a l l} \pm \sigma$ \\
ADJ & $0.954 \pm 0.005$ & $0.952 \pm 0.004$ & $0.957 \pm 0.005$ \\
ADV & $0.929 \pm 0.003$ & $0.936 \pm 0.008$ & $0.919 \pm 0.009$ \\
ADV-KS & $0.644 \pm 0.030$ & $0.706 \pm 0.056$ & $0.600 \pm 0.062$ \\
ADV-KS-REL & $0.860 \pm 0.013$ & $0.853 \pm 0.020$ & $0.868 \pm 0.013$ \\
ART & $0.990 \pm 0.000$ & $0.986 \pm 0.005$ & $0.990 \pm 0.000$ \\
CUR & $1.000 \pm 0.000$ & $1.000 \pm 0.000$ & $1.000 \pm 0.000$ \\
IN & $0.705 \pm 0.046$ & $0.780 \pm 0.119$ & $0.650 \pm 0.000$ \\
KC & $0.980 \pm 0.000$ & $0.991 \pm 0.003$ & $0.972 \pm 0.004$ \\
KS & $0.910 \pm 0.007$ & $0.917 \pm 0.011$ & $0.906 \pm 0.013$ \\
N & $0.980 \pm 0.000$ & $0.980 \pm 0.000$ & $0.975 \pm 0.005$ \\
NPROP & $0.970 \pm 0.000$ & $0.969 \pm 0.006$ & $0.972 \pm 0.006$ \\
NUM & $0.970 \pm 0.000$ & $0.970 \pm 0.009$ & $0.968 \pm 0.008$ \\
PCP & $0.970 \pm 0.000$ & $0.963 \pm 0.005$ & $0.976 \pm 0.005$ \\
PDEN & $0.902 \pm 0.006$ & $0.907 \pm 0.016$ & $0.892 \pm 0.010$ \\
PREP & $0.980 \pm 0.000$ & $0.980 \pm 0.000$ & $0.982 \pm 0.004$ \\
PREP+ADV & $0.940 \pm 0.021$ & $0.936 \pm 0.034$ & $0.944 \pm 0.039$ \\
PREP+ART & $0.990 \pm 0.000$ & $0.990 \pm 0.000$ & $0.992 \pm 0.004$ \\
PREP+PRO-KS & $0.099 \pm 0.159$ & $0.150 \pm 0.242$ & $0.075 \pm 0.121$ \\
PREP+PRO-KS-REL & $1.000 \pm 0.000$ & $1.000 \pm 0.000$ & $1.000 \pm 0.000$ \\
PREP+PROADJ & $1.000 \pm 0.000$ & $0.990 \pm 0.000$ & $1.000 \pm 0.000$ \\
PREP+PROPESS & $1.000 \pm 0.000$ & $1.000 \pm 0.000$ & $1.000 \pm 0.000$ \\
PREP+PROSUB & $0.839 \pm 0.016$ & $0.867 \pm 0.022$ & $0.814 \pm 0.035$ \\
PRO-KS & $0.755 \pm 0.015$ & $0.726 \pm 0.055$ & $0.795 \pm 0.066$ \\
PRO-KS-REL & $0.926 \pm 0.005$ & $0.903 \pm 0.014$ & $0.952 \pm 0.006$ \\
PROADJ & $0.970 \pm 0.000$ & $0.968 \pm 0.004$ & $0.973 \pm 0.005$ \\
PROPESS & $0.982 \pm 0.004$ & $0.989 \pm 0.003$ & $0.980 \pm 0.000$ \\
PROSUB & $0.903 \pm 0.005$ & $0.906 \pm 0.021$ & $0.900 \pm 0.016$ \\
PU & $1.000 \pm 0.000$ & $1.000 \pm 0.000$ & $1.000 \pm 0.000$ \\
VAUX & $0.987 \pm 0.005$ & $0.989 \pm 0.003$ & $0.982 \pm 0.004$ \\
Média \pm & $\mathbf{0 . 9 0 2} \pm \mathbf{0 . 0 1 1}$ & $\mathbf{0 . 9 0 8} \pm \mathbf{0 . 0 2 2}$ & $\mathbf{0 . 8 9 9} \pm \mathbf{0 . 0 1 4}$
\end{tabular}

$\mathrm{O}$ corpus Tycho Brahe obteve a pior avaliação média $\left(F_{1}=0.740\right.$, precision $=0.753$ e recall $=0.743)$ se comparado aos outros corpora. Esse corpus tem um sistema de etiquetas muito mais granular, são 265 classes diferente. E como podemos ver no Apêndice A, muitas etiquetas apresentam uma baixa representação, algumas delas contam com menos de uma dezena de exemplos em todo o corpus. E as etiquetas com baixa representação tendem a obter resultados piores, fazendo com que a média de $F_{1}$ seja possivelmente "puxada" para baixo por essas classes.

Os valores de $F_{1}$, precision e recall médio de todas as classes presentes no conjunto de teste do corpus Tycho Brahe, estão detalhados no Apêndice B. Em 
geral, as classes com uma boa representação tiveram um bom desempenho. Na Figura 6.14 mostramos os valores de $F_{1}$ para as 15 etiquetas com as maiores representações no corpus Tycho Brahe, a maioria das classes alcançaram valor de $F_{1}$ entorno de $98 \%$. Para melhor entender o desempenho de todas as 233 etiquetas presentes no conjunto de teste, montamos um histograma de frequência (Figura 6.15) das etiquetas em 5 grupos avaliação. A maioria das etiquetas (158) obtiveram um valor de $F_{1}$ entre $80 \%$ e 100\%. 38 etiquetas foram avaliadas com um $F_{1}$ entre $0 \%$ e $20 \%$. Embora tenhamos algumas dezenas de etiquetas com um pobre desempenho ainda temos a maioria delas com uma boa avaliação.

No corpus Mac-Morpho v1 e no corpus Mac-Morpho v2 notamos que as classes com os piores desempenhos apresentavam uma baixa representatividade de exemplo. Para o corpus Tycho Brahe, que têm 265 classes diferentes, montamos um gráfico para investigar se existe algum tipo de correlação entre a grandeza do número de exemplos de uma classe e seu desempenho em $F_{1}$. Na Figura 6.16 apresentamos um gráfico de correlação, no eixo $y$ temos o logaritmo do número de exemplo da uma classe, e no eixo $x$ temos o desempenho da classe em $F_{1}$. Calculamos o coeficiente de correlação de Pearson das duas variáveis e descobrimos uma correlação positiva de $p=0.701$.

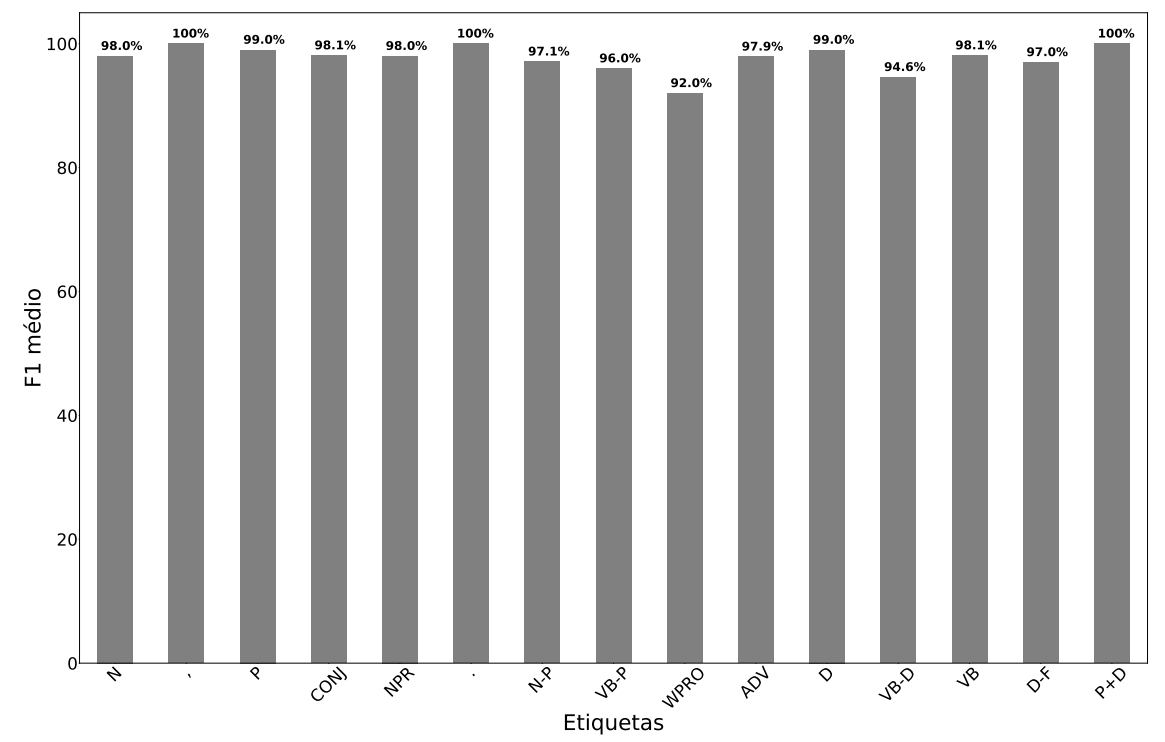

Figura 6.14: Valor médio de $F_{1}$ dos 10 experimentos realizados com o modelo BLSTM-WE-CE no corpus TychoBrahe para as 15 etiquetas mais frequentes. 


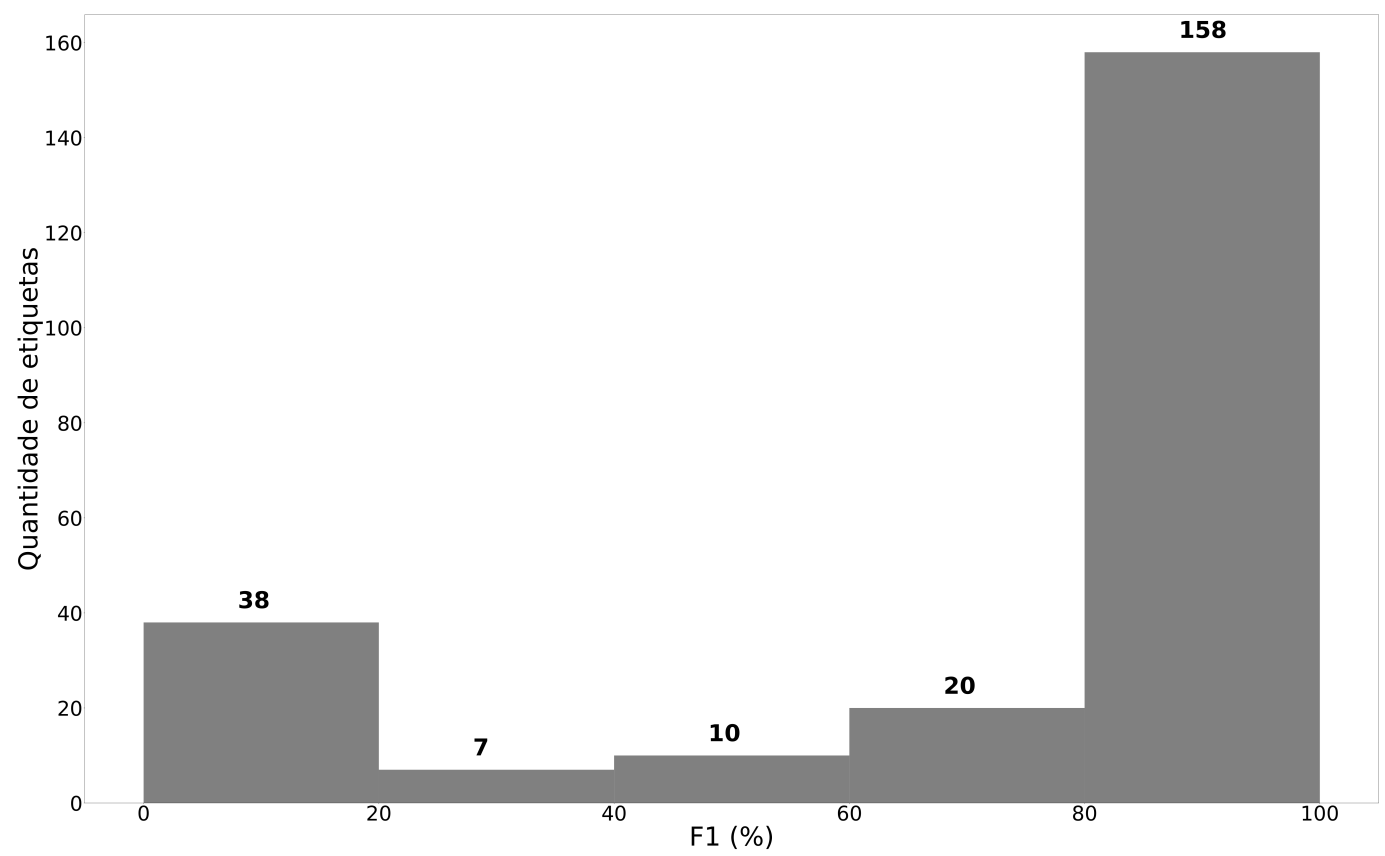

Figura 6.15: Histograma: quantidade de etiquetas em 5 grupo de avaliação de $F_{1}$

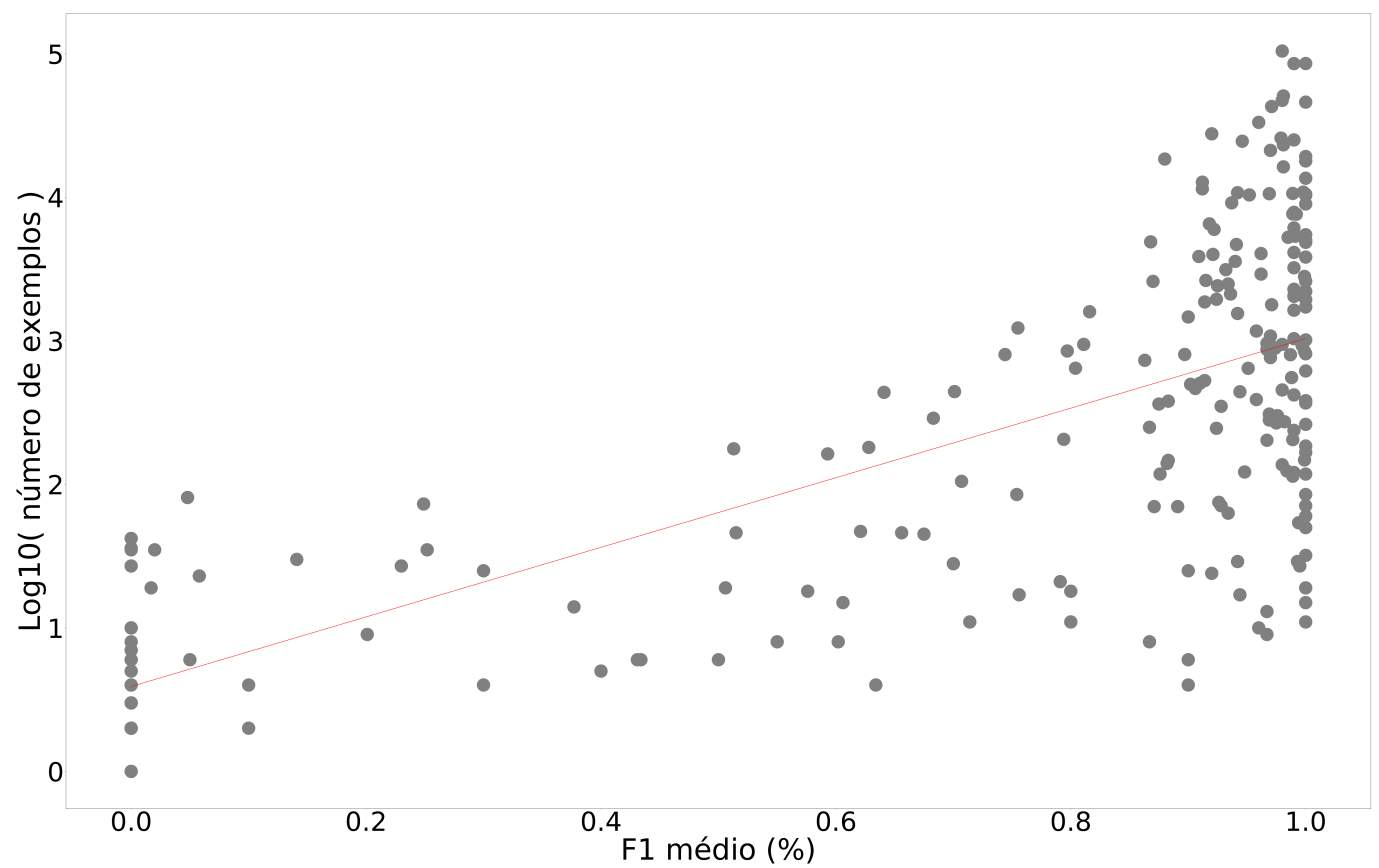

Figura 6.16: Gráfico de correlação do valor $F_{1}$ da etiqueta pelo logaritmo do número de exemplos. 


\section{7}

\section{Análise de erro}

Para a nossa arquitetura BLSTM-WE-CE, analisamos nos três corpora os erros mais frequentes. Nas Tabelas 6.18, 6.19 e 6.20 temos as palavras que mais obtiveram erros de marcação e as etiquetas que elas possuem no conjunto de teste, respectivamente para os corpora Mac-Morpho v1, Mac-Morpho v2 e Tycho Brahe.

As palavras mais difíceis de se atribuir etiquetas morfossintáticas são "que" e "a", tanto para o Português Contemporâneo, quanto para o Português Histórico. Essas duas palavras apareceram no topo das tabelas de erros dos três corpora. A complexidade de marcação, e consequentemente a maior quantidade de erros nessas palavras pode ser atribuída, provavelmente, ao grande número de classes que as palavras "que" e "a" podem ser relacionadas no conjunto de dados.

No corpus Mac-Morpho v1, a palavra com o maior número de diferentes classes associadas, é justamente a palavra "que", com 17 classes. A segunda é a palavra "a", com 14 classes (em Figura 6.2). O mesmo acontece no corpus Mac-Morpho v2, a palavra "que", tem 16 diferentes classes associadas, o maior número de todo corpus, e a palavra "a" tem 13, o segundo maior número de classes associadas. O mesmo vale para o corpus de Português Histórico (Tycho Brahe), a palavra "que", com 16 classes. A segunda é a palavra "a", com 13 classes, sendo essas a duas palavras com mais classes nesse corpus.

Também encontramos no topo das tabelas de erros, palavras como "de", "o", "se", "um", "nem", "como" e "até". Por conta da ambiguidade presente na linguagem humana, essas palavras também assumem diferentes classes morfossintática de acordo com o contexto em que são usadas.

\section{8}

\section{Análise de character embedding}

Nessa subseção realizamos uma análise nos vetores de character embedding gerados durante o treino do modelo BLSTM-WE-CE com o corpus MacMorpho v1. Tais vetores, geram representações para as palavras do conjunto de treino por meio de uma camada BLSTM. Essas representações são capazes de capturar informações morfológicas de, por exemplo, tamanho, prefixo, sufixo e vocabulário de caracteres.

Nós computamos a similaridade de cosseno de algumas palavras $w_{\text {oov }}$, que estão fora do vocabulário de treino, para as palavras $w_{v}$, que fizeram parte do treino do modelo. Na Tabela 6.21, apresentamos as palavras $w_{\text {oov }}$ e as 5 palavras $w_{v}$ mais próximas, segundo suas respectivas similaridades de cosseno. 
Tabela 6.18: Os tokens com mais erros e as etiquetas que eles possuem no conjunto de teste do Mac-Morpho v1.

\begin{tabular}{lll}
\hline Palavra & Etiquetas & $\begin{array}{c}\text { \# de } \\
\text { erros }\end{array}$ \\
\hline que & NPROP, ADV-KS, PRO-KS-REL, ADV, PROADJ, ADV- & 281 \\
& KS-REL, PROSUB, PREP, PRO-KS, KS, PDEN & \\
a & N, KC, PRO-KS-REL, PROADJ, ADV, ART, PROSUB, & 234 \\
& PREP, NPROP, KS, PROPESS & \\
de & N, KC, ADV, PREP, NPROP, KS, PDEN & 162 \\
o & NPROP, ADV-KS, PRO-KS-REL, ADV, PROADJ, ART, & 123 \\
& PROSUB, PRO-KS, KS, PROPESS & \\
como & KC, NPROP, ADV-KS, ADV-KS-REL, PREP, ADV, KS & 92 \\
um & N, NPROP, NUM, PROADJ, ART, PROSUB, ADV & 70 \\
até & NPROP, PREP, ADV, KS, PDEN & 64 \\
se & NPROP, KS, PROPESS & 41 \\
é & V, VAUX, PROSUB, NPROP, PDEN & 40 \\
uma & N, NUM, ADV, ART, PROSUB, PREP, NPROP, KS & 39 \\
\hline
\end{tabular}

Tabela 6.19: Os tokens com mais erros e as etiquetas que eles possuem no conjunto de teste do Mac-Morpho v2.

\begin{tabular}{lll}
\hline Palavra & Etiquetas & $\begin{array}{c}\text { \# de } \\
\text { erros }\end{array}$ \\
\hline que & NPROP, ADV-KS, PRO-KS-REL, PROADJ, ADV-KS- & 154 \\
& REL, ADV, PROSUB, PRO-KS, KS, PDEN & \\
a & KC, PRO-KS-REL, PROADJ, ADV, ART, PROSUB, & 95 \\
& PREP, NPROP, PROPESS & 67 \\
de & N, ADV, PREP, NPROP, KS, PDEN & 63 \\
o & PRO-KS-REL, ADV, ART, PRO-KS, PROSUB, NPROP, & 53 \\
& PROPESS & 34 \\
mais & N, KC, NPROP, PROADJ, PROSUB, PREP, ADV & 33 \\
como & KC, ADV-KS, ADV, ADV-KS-REL, PREP, NPROP, KS & 28 \\
até & ADV, KS, PDEN, PREP & 28 \\
do & N, NUM, ADV, PROADJ, ART, PROSUB, NPROP & 21 \\
da & N, PREP+ART, NPROP, KS, PREP+PROSUB & 20
\end{tabular}

Com a Tabela 6.21 podemos notar a capacidade dos vetores de character embedding gerado com BLSTM de capturar prefixos e sufixos. Para a palavra fora do vocabulário "imprecisão", por exemplo, temos palavras próximas com prefixos parecidos ( "im", "i") e também sufixos parecidos ("são", "ão"). Para a palavras "recrutada", vemos também semelhanças de prefixos e sufixos com as palavras próximas, e também uma semelhança de tamanho, todas a palavras têm 9 caracteres. Podemos notar essas semelhanças para a outras palavras $w_{\text {oov }}$ da tabela. 
Tabela 6.20: Os tokens com mais erros e as etiquetas que eles possuem no conjunto de teste do Tycho Brahe.

\begin{tabular}{lll}
\hline Palavra & Etiquetas & \# de erros \\
\hline que & CONJ, WPRO, FW, WD, C, D, CONJS, WD-P & 1208 \\
a & N, CL, FW, D, NPR, P, D-F & 230 \\
se & WQ, CONJ, FW, WD, CONJS, SE & 189 \\
porque & WADV, CONJ, P+WPRO, CONJS & 127 \\
como & WADV, CONJ, WPRO, WD, CONJS, VB-P & 90 \\
nem & WADV, CONJ, NEG, P, CONJ-NEG & 73 \\
o & N, CL, FW, D, NPR, P & 63 \\
as & D-P, CL, D-F-P, D-F, P & 55 \\
foi & VB-D, SR-D & 42 \\
até & P, FP & 31 \\
\hline
\end{tabular}

Tabela 6.21: Cinco palavras fora do vocabulário e suas palavras mais similares de acordo com a representação de character embedding

\begin{tabular}{llll}
\hline imprecisão & recrutada & pré-requisito & seccional \\
\hline iluminação & rejeitada & pré-campanhas & seleciona \\
imaginação & resgatado & pré-suposições & semifinal \\
insinuação & recontada & pernambucano & sertaneja \\
tranmissão & resultado & curta-metragem & sensorial \\
implicação & recortada & pressupostos & soluciona \\
\hline
\end{tabular}




\section{Conclusões}

\section{1}

\section{Resumo do trabalho}

Nessa dissertação, desenvolvemos um etiquetador morfossintático (POS Tagger) para a Língua Portuguesa, usando redes neurais recorrentes. Nossa arquitetura se baseia no trabalho de Ma e Hovy (25), que apresentou em 2016 o POS Tagger estado da arte para a língua Inglesa, e no trabalho de Marulli et al. (46) que apresentou em 2018 o POS Tagger estado da arte para Língua Italiana.

A arquitetura do nosso POS Tagger de Língua Portuguesa, não depende de features manuais, ou features específicas do domínio ou dados externos ao conjunto de treino. Usamos uma rede neural recorrente BLSTM para resolver o problema de POS Tagging para o Português. E que tem como entrada representações de word embedding carregados de modelos pré-treinados, combinados a representações de character embedding gerados por outra camada de BLSTM.

Aplicamos a arquitetura BLSTM-WE-CE para POS Tagging em três corpora de Língua Portuguesa. Em todas os três corpora apresentamos um desempenho em acurácia e acurácia FV ligeiramente superior ao estado da arte reportado por Fonseca et al. (45) e Santos e Zadrozny (44). Obtivemos para o corpus Mac-Morpho v1, $97.83 \%$ de acurácia em média e $95.48 \%$ de acurácia FV em média. Para o corpus Mac-Morpho v2, 97.65\% de acurácia em média e $95.23 \%$ de acurácia FV em média. E para o corpus Tycho Brahe obtivemos, $97.35 \%$ de acurácia em média e $87.40 \%$ de acurácia FV em média.

Avaliamos ainda separadamente o desempenho de cada etiqueta nos três corpora com as medidas de $F_{1}$-score, precision e recall. Notamos que as etiquetas com um baixo número de exemplos no conjunto de treino (baixa representatividade), apresentam em muitos casos, um baixo desempenho em $F_{1}$-score. Porém, a maioria das etiquetas nos três corpora apresentam uma boa avaliação, em torno de $90 \%$ de $F_{1}$-score. Tiramos a média das etiquetas por corpus e vimos que o corpus Mac-Morpho v1 obteve a melhor avaliação média $\left(F_{1}=0.948\right.$, precision $=0.953$, recall $\left.=0.945\right)$, seguido do corpus Mac-Morpho v2 
$\left(F_{1}=0.902\right.$, precision $=0.908$ e recall $\left.=0.899\right)$ e corpus Tycho Brahe $\left(F_{1}=0.740\right.$, precision $=0.753$ e recall $=0.743)$.

\section{2}

\section{Contribuições}

Nesse trabalho demonstramos empiricamente que a arquitetura proposta (BLSTM-WE-CE), que usa uma rede BLSTM para classificar as etiquetas, e uma outra rede BLSTM para gerar representações de character embedding, é bem adequada para treinar POS Taggers para a Língua Portuguesa, tanto para o Português Contemporâneo, quando para o Português Histórico.

Criamos POS Taggers para a Língua Portuguesa que superam ligeiramente o estado da arte em termos de acurácia e acurácia FV. Avaliamos três corpora diferentes, o Mac-Morpho v1 (Ac. $=97.83 \%$, Ac. FV=95.48) o MacMorpho v2 (Ac. $=97.65 \%$, Ac. FV=95.23), que são compostos de sentenças do Português Contemporâneo. E ainda o corpus Tycho Brahe (Ac.=97.35, Ac. $\mathrm{FV}=87.40)$, composto de sentenças do Português Histórico.

Realizamos ainda um estudo empírico para descobrir qual dentre os mais populares algoritmos de criação word embedding, é mais adequado para a atividade POS Tagging em Português. Analisamos os modelos Glove e Word2Vec, FastText e Wang2Vec em suas duas versões (CBOW e Skip-Gram). O modelo Wang2Vec apresentou o melhor desempenho em acurácia para os três corpora analisadas. O bom desempenho de modelo Wang2vec, pode vir provavelmente, de seu foco na captura de informações sintáticas, que leva em conta a ordem em que as palavras aparecem, o que não é feito pelo os outros modelos. E esse tipo de informação se mostrou bastante útil para a classificação morfossintática em Português com nossa arquitetura de rede neural.

\section{3}

\section{Trabalhos futuros}

Existem algumas possibilidades de trabalhos futuros. Poderíamos aplicar a arquitetura apresentada nesse trabalho em outras atividades de PLN em Português, como exemplo reconhecimento de entidades nomeadas em diversos domínios (em textos de redes sociais, no meio jurídico e em aplicações da indústria de óleo de gás).

Nessa dissertação investigamos o uso de diferentes modelos de word embeddings (Word2Vec, FastText, Wang2Vec e Glove) no contexto da atividade de POS Tagging. Recentemente, em 2018 Peters et al. (71) propuseram uma nova técnica para gerar representações de word embeddings, o chamado ELMo (Embeddings from Language Models). Diferentes dos modelos investigados nesse 
trabalho, o modelo ELMo gerar representações vetoriais contextualizadas para as palavras, portanto a mesma palavra pode ter diferentes vetores dependendo do contexto que é usada. Essa técnica melhorou significativamente o estado da arte em uma ampla variedade de problemas de PLN. Uma possibilidade de trabalho futuro seria aplicar os vetores de embeddings contextualizados ELMo na arquitetura de POS tagging em Português.

No campo da visão computacional, os pesquisadores mostraram repetidamente o valor do chamado transfer learning. Em que redes neurais são treinas para uma certa atividade usando grande bancos de imagens. Então os pesos gerados por essas redes são usados como um basis inicial para treinar novas redes em outras atividades especificas.

Também no ano de 2018, pesquisados do Google AI Language propuseram o BERT (72) (Bidirectional Encoder Representations from Transformers), um modelo que pode ser usado como pré-treino para redes neurais em atividades de PLN, realizando o transfer learning. Com o BERT os pesquisados alcançaram o estado da arte em 11 atividade de PLN. Uma possibilidade de trabalho futuro seria estudar o impacto do uso do modelo BERT em atividades de PLN em Português. 


\section{Referências bibliográficas}

[1] BOHNET, B.; MCDONALD, R.; SIMÕES, G.; ANDOR, D.; PITLER, E. ; MAYNEZ, J.. Morphosyntactic tagging with a meta-bilstm model over context sensitive token encodings. In: PROCEEDINGS OF THE 56TH ANNUAL MEETING OF THE ASSOCIATION FOR COMPUTATIONAL LINGUISTICS (VOLUME 1: LONG PAPERS), volumen 1, p. 2642-2652, 2018.

[2] CHOWDHURY, G. G.. Natural language processing. Annual review of information science and technology, 37(1):51-89, 2003.

[3] COPPIN, B.. Artificial intelligence illuminated. Jones and Bartlett Publishers, 2004.

[4] RANJAN, N.; MUNDADA, K.; PHALTANE, K. ; AHMAD, S.. A survey on techniques in nlp. International Journal of Computer Applications, 134(8):6-9, 2016.

[6] HIRSCHBERG, J.; MANNING, C. D.. Advances in natural language processing. Science, 349(6245):261-266, 2015.

[7] MARR, B.. How much data do we create every day? the mindblowing stats everyone should read, 2018.

[8] GO, A.; BHAYANI, R. ; HUANG, L.. Twitter sentiment classification using distant supervision. CS224N Project Report, Stanford, 1(12), 2009.

[9] DE HOLANDA MAIA, M. R.; XEXÉO, G. B.. Part-of-speech tagging of portuguese using hidden markov models with character language model emissions. In: PROCEEDINGS OF THE 8TH BRAZILIAN SYMPOSIUM IN INFORMATION AND HUMAN LANGUAGE TECHNOLOGY, 2011.

[10] ZHANG, D.; LEE, W. S.. Question classification using support vector machines. In: PROCEEDINGS OF THE 26TH ANNUAL INTERNATIONAL ACM SIGIR CONFERENCE ON RESEARCH AND DEVELOPMENT IN INFORMAION RETRIEVAL, p. 26-32. ACM, 2003. 
[11] GOLDBERG, Y.. A primer on neural network models for natural language processing. Journal of Artificial Intelligence Research, 57:345420, 2016.

[12] GIMPEL, K.; SCHNEIDER, N.; O'CONNOR, B.; DAS, D.; MILLS, D.; ElSENSTEIN, J.; HEILMAN, M.; YOGATAMA, D.; FLANIGAN, J. ; SMITH, N. A.. Part-of-speech tagging for twitter: Annotation, features, and experiments. In: PROCEEDINGS OF THE 49TH ANNUAL MEETING OF THE ASSOCIATION FOR COMPUTATIONAL LINGUISTICS: HUMAN LANGUAGE TECHNOLOGIES: SHORT PAPERS-VOLUME 2, p. 42-47. Association for Computational Linguistics, 2011.

[13] GIMÉNEZ, J.; MARQUEZ, L.. Svmtool: A general pos tagger generator based on support vector machines. In: IN PROCEEDINGS OF THE 4TH INTERNATIONAL CONFERENCE ON LANGUAGE RESOURCES AND EVALUATION. Citeseer, 2004.

[14] DAS, O.; BALABANTARAY, R. C.. Sentiment analysis of movie reviews using pos tags and term frequencies. International Journal of Computer Applications, 96(25), 2014.

[15] MA, J.; LIU, H.; HUANG, D. ; SHENG, W.. An english part-of-speech tagger for machine translation in business domain. In: NATURAL LANGUAGE PROCESSING ANDKNOWLEDGE ENGINEERING (NLP-KE), 2011 7TH INTERNATIONAL CONFERENCE ON, p. 183-189. IEEE, 2011.

[16] WANG, W.; AUER, J.; PARASURAMAN, R.; ZUBAREV, I.; BRANDYBERRY, D. ; HARPER, M.. A question answering system developed as a project in a natural language processing course. In: PROCEEDINGS OF THE 2000 ANLP/NAACL WORKSHOP ON READING COMPREHENSION TESTS AS EVALUATION FOR COMPUTER-BASED LANGUAGE UNDERSTANDING SYTEMS-VOLUME 6, p. 28-35. Association for Computational Linguistics, 2000.

[17] SCHMIDHUBER, J.. Deep learning in neural networks: An overview. Neural networks, 61:85-117, 2015.

[18] HATCHER, W. G.; YU, W.. A survey of deep learning: Platforms, applications and emerging research trends. IEEE Access, 6:2441124432, 2018. 
[19] LIU, W.; WANG, Z.; LIU, X.; ZENG, N.; LIU, Y. ; ALSAADI, F. E.. A survey of deep neural network architectures and their applications. Neurocomputing, 234:11-26, 2017.

[20] ABRAHAM, A.. Artificial neural networks. handbook of measuring system design, 2005.

[22] HOCHREITER, S.; SCHMIDHUBER, J.. Long short-term memory. Neural computation, 9(8):1735-1780, 1997.

[23] GOOdFElloW, I.; BEnGIO, Y. ; COURVILlE, A.. Deep Learning. MIT Press, 2016. http://www. deeplearningbook.org.

[24] LECUN, Y.; BENGIO, Y. ; HINTON, G.. Deep learning. nature, 521(7553):436, 2015.

[25] MA, X.; HOVY, E.. End-to-end sequence labeling via bi-directional lstm-cnns-crf. In: PROCEEDINGS OF THE 54TH ANNUAL MEETING OF THE ASSOCIATION FOR COMPUTATIONAL LINGUISTICS (VOLUME 1: LONG PAPERS), volumen 1, p. 1064-1074, 2016.

[26] YOUNG, T.; HAZARIKA, D.; PORIA, S. ; CAMBRIA, E.. Recent trends in deep learning based natural language processing. ieee Computational intelligenCe magazine, 13(3):55-75, 2018.

[27] HARTMANN, N.; FONSECA, E.; SHULBY, C.; TREVISO, M.; SILVA, J. ; ALUÍSIO, S.. Portuguese word embeddings: Evaluating on word analogies and natural language tasks. In: PROCEEDINGS OF THE 11TH BRAZILIAN SYMPOSIUM IN INFORMATION AND HUMAN LANGUAGE TECHNOLOGY, p. 122-131, 2017.

[28] LAI, S.; LIU, K.; HE, S. ; ZHAO, J.. How to generate a good word embedding. IEEE Intelligent Systems, 31(6):5-14, 2016.

[29] BENGIO, Y.; DUCHARME, R.; VINCENT, P. ; JAUVIN, C.. A neural probabilistic language model. Journal of machine learning research, 3(Feb):1137-1155, 2003.

[31] PENNINGTON, J.; SOCHER, R. ; MANNING, C.. Glove: Global vectors for word representation. In: PROCEEDINGS OF THE 2014 CONFERENCE ON EMPIRICAL METHODS IN NATURAL LANGUAGE PROCESSING (EMNLP), p. 1532-1543, 2014. 
[32] MIKOLOV, T.; CHEN, K.; CORRADO, G. S. ; DEAN, J.. Efficient estimation of word representations in vector space. CoRR, abs/1301.3781, 2013.

[33] BOJANOWSKI, P.; GRAVE, E.; JOULIN, A. ; MIKOLOV, T.. Enriching word vectors with subword information. Transactions of the Association for Computational Linguistics, 5:135-146, 2017.

[34] LING, W.; DYER, C.; BLACK, A. W. ; TRANCOSO, I.. Two/too simple adaptations of word2vec for syntax problems. In: PROCEEDINGS OF THE 2015 CONFERENCE OF THE NORTH AMERICAN CHAPTER OF THE ASSOCIATION FOR COMPUTATIONAL LINGUISTICS: HUMAN LANGUAGE TECHNOLOGIES, p. 1299-1304. Association for Computational Linguistics, 2015.

[35] DICKINSON, B.; HU, W.. Sentiment analysis of investor opinions on twitter. Social Networking, 4(03):62, 2015.

[36] DOS SANTOS, C.; GATTI, M.. Deep convolutional neural networks for sentiment analysis of short texts. In: PROCEEDINGS OF COLING 2014, THE 25TH INTERNATIONAL CONFERENCE ON COMPUTATIONAL LINGUISTICS: TECHNICAL PAPERS, p. 69-78, 2014.

[37] KIM, Y.; JERNITE, Y.; SONTAG, D. ; RUSH, A. M.. Character-aware neural language models. In: AAAI, p. 2741-2749, 2016.

[38] GHAZVININEJAD, M.; SHI, X.; CHOI, Y. ; KNIGHT, K.. Generating topical poetry. In: PROCEEDINGS OF THE 2016 CONFERENCE ON EMPIRICAL METHODS IN NATURAL LANGUAGE PROCESSING, p. 11831191, 2016.

[39] VENUGOPALAN, S.; XU, H.; DONAHUE, J.; ROHRBACH, M.; MOONEY, R. ; SAENKO, K.. Translating videos to natural language using deep recurrent neural networks. In: PROCEEDINGS OF THE 2015 CONFERENCE OF THE NORTH AMERICAN CHAPTER OF THE ASSOCIATION FOR COMPUTATIONAL LINGUISTICS: HUMAN LANGUAGE TECHNOLOGIES, p. 1494-1504, 2015.

[40] CORRÊA, D. C.; SAlVADEO, D. H.; LEVADA, A. L.; SAITO, J. H. ; DA, N.. Using lstm network in face classification problems, 2008.

[41] GRAVES, A.; SCHMIDHUBER, J.. Framewise phoneme classification with bidirectional lstm and other neural network architectures. Neural Networks, 18(5-6):602-610, 2005. 
[42] COLLOBERT, R.; WESTON, J.; BOTTOU, L.; KARLEN, M.; KAVUKCUOGLU, K. ; KUKSA, P.. Natural language processing (almost) from scratch. Journal of Machine Learning Research, 12(Aug):2493-2537, 2011.

[43] FernandeS, E. R.; RODRIGUeS, I. M. ; MILIDIÚ, R. L.. Portuguese part-of-speech tagging with large margin structure learning. In: INTELLIGENT SYSTEMS (BRACIS), 2014 BRAZILIAN CONFERENCE ON, p. 25-30. IEEE, 2014.

[44] DOS SANTOS, C. N.; ZADROZNY, B.. Training state-of-the-art portuguese pos taggers without handcrafted features. In: INTERNATIONAL CONFERENCE ON COMPUTATIONAL PROCESSING OF THE PORTUGUESE LANGUAGE, p. 82-93. Springer, 2014.

[45] FONSECA, E. R.; ROSA, J. L. G. ; ALUÍSIO, S. M.. Evaluating word embeddings and a revised corpus for part-of-speech tagging in portuguese. Journal of the Brazilian Computer Society, 21(1):2, 2015.

[46] MARULLI, F.; POTA, M. ; ESPOSITO, M.. A comparison of character and word embeddings in bidirectional lstms for pos tagging in italian. In: INTERNATIONAL CONFERENCE ON INTELLIGENT INTERACTIVE MULTIMEDIA SYSTEMS AND SERVICES, p. 14-23. Springer, 2018.

[47] ALUÍSIO, S.; PELIZZONI, J.; MARCHI, A. R.; DE OLIVEIRA, L.; MANENTI, R. ; MARQUIAFÁVEL, V.. An account of the challenge of tagging a reference corpus for brazilian portuguese. In: INTERNATIONAL WORKSHOP ON COMPUTATIONAL PROCESSING OF THE PORTUGUESE LANGUAGE, p. 110-117. Springer, 2003.

[48] FONSECA, E. R.; ROSA, J. L. G.. Mac-morpho revisited: Towards robust part-of-speech tagging. In: PROCEEDINGS OF THE 9TH BRAZILIAN SYMPOSIUM IN INFORMATION AND HUMAN LANGUAGE TECHNOLOGY, 2013.

[49] TEMPONI, C. N.; OTHERS. O corpus anotado do português histórico: um avanço para as pesquisas em lingüística histórica do português. Revista Virtual de Estudos da Linguagem: ReVEL, 2(3):1, 2004.

[50] CAMBRIA, E.; WHITE, B.. Jumping nlp curves: A review of natural language processing research. IEEE Computational intelligence magazine, 9(2):48-57, 2014. 
[51] JUNG, S.; LEE, C. ; HWANG, H.. End-to-end korean part-of-speech tagging using copying mechanism. ACM Transactions on Asian and Low-Resource Language Information Processing (TALLIP), 17(3):19, 2018.

[52] WANG, P.; QIAN, Y.; SOONG, F. K.; HE, L. ; ZHAO, H.. Part-of-speech tagging with bidirectional long short-term memory recurrent neural network. arXiv preprint arXiv:1510.06168, 2015.

[53] SRINIVASA, K.; ANUPINDI, S.; SHARATH, R. ; CHAITANYA, S. K.. Analysis of facial expressiveness captured in reaction to videos. In: 2017 IEEE 7TH INTERNATIONAL ADVANCE COMPUTING CONFERENCE (IACC), p. 664-670. IEEE, 2017.

[54] SCHUSTER, M.; PALIWAL, K. K.. Bidirectional recurrent neural networks. IEEE Transactions on Signal Processing, 45(11):2673-2681, 1997.

[55] ZHANG, Q.; YANG, L. T.; CHEN, Z. ; LI, P.. A survey on deep learning for big data. Information Fusion, 42:146-157, 2018.

[56] OLAH, C.. Understanding lstm networks, 2015.

[57] DENG, L.; LIU, Y.. Deep Learning in Natural Language Processing. Springer, 2018.

[58] GUPTA, S.. Word embeddings in nlp and its applications, 2019.

[59] RONG, X.. word2vec parameter learning explained. arXiv preprint arXiv:1411.2738, 2014.

[60] KARANI, D.. Introduction to word embedding and word2vec, 2018.

[61] FINGER, M.. Técnicas de otimizaçao da precisao empregadas no etiquetador tycho brahe. Proceedings of PROPOR, Sao Paulo, p. 141154,2000 .

[62] BRILL, E. D.. A corpus-based approach to language learning. IRCS Technical Reports Series, p. 191, 1993.

[63] BRILL, E.. Transformation-based error-driven learning and natural language processing: A case study in part-of-speech tagging. Computational linguistics, 21(4):543-565, 1995.

[64] KEPLER, F. N.; FINGER, M.. Part-of-speech tagging of portuguese based on variable length markov chains. In: INTERNATIONAL 
WORKSHOP ON COMPUTATIONAL PROCESSING OF THE PORTUGUESE LANGUAGE, p. 248-251. Springer, 2006.

[65] DOS SANTOS, C. N.; MILIDIÚ, R. L. ; RenTERÍA, R. P.. Portuguese part-of-speech tagging using entropy guided transformation learning. In: INTERNATIONAL CONFERENCE ON COMPUTATIONAL PROCESSING OF THE PORTUGUESE LANGUAGE, p. 143-152. Springer, 2008.

[66] SRIVASTAVA, N.; HINTON, G.; KRIZHEVSKY, A.; SUTSKEVER, I. ; SALAKHUTDINOV, R.. Dropout: a simple way to prevent neural networks from overfitting. The Journal of Machine Learning Research, 15(1):1929-1958, 2014.

[68] KINGMA, D. P.; BA, J.. Adam: a method for stochastic optimization. iclr (2015), 2015.

[69] Peters, M.; AMmar, W.; Bhagavatula, C. ; POWeR, R.. Semisupervised sequence tagging with bidirectional language models. In: PROCEEDINGS OF THE 55TH ANNUAL MEETING OF THE ASSOCIATION FOR COMPUTATIONAL LINGUISTICS (VOLUME 1: LONG PAPERS), p. 1756-1765, 2017.

[70] CHIU, J. P.; NICHOLS, E.. Named entity recognition with bidirectional lstm-cnns. Transactions of the Association for Computational Linguistics, 4:357-370, 2016.

[71] PETERS, M. E.; NEUMANN, M.; IYYER, M.; GARDNER, M.; CLARK, C.; LEE, K. ; ZETTLEMOYER, L.. Deep contextualized word representations. In: PROCEEDINGS OF NAACL-HLT, p. 2227-2237, 2018.

[72] DeVlin, J.; ChANG, M.-W.; LEE, K. ; TOUTANOVA, K.. Bert: Pretraining of deep bidirectional transformers for language understanding. In: PROCEEDINGS OF THE 2019 CONFERENCE OF THE NORTH AMERICAN CHAPTER OF THE ASSOCIATION FOR COMPUTATIONAL LINGUISTICS: HUMAN LANGUAGE TECHNOLOGIES, VOLUME 1 (LONG AND SHORT PAPERS), p. 4171-4186, 2019. 
A

\section{Descrição das etiquetas do Tycho Brahe corpus}

Este apêndice contém tabelas com informações de descrição e distribuição das 265 etiquetas do Tycho Brahe corpus descrito no Capítulo 6 desta dissertação. A Tabela A.1 mostra a distribuição das etiquetas nos conjuntos de treino, desenvolvimento e teste.

Tabela A.1: Distribuição das etiquetas no corpus Tycho Brahe

\begin{tabular}{|c|c|c|c|c|}
\hline Etiquetas & Treino & Desen. & Teste & Total \\
\hline $\mathrm{N}$ & 74197 & 4102 & 26300 & $104599(10.1 \%)$ \\
\hline & 60818 & 3370 & 21447 & $85635(8.27 \%)$ \\
\hline $\mathrm{P}$ & 60610 & 3430 & 21465 & $85505(8.26 \%)$ \\
\hline CONJ & 35923 & 2048 & 12879 & $50850(4.91 \%)$ \\
\hline NPR & 33743 & 1763 & 11886 & $47392(4.58 \%)$ \\
\hline & 32809 & 1746 & 11520 & $46075(4.45 \%)$ \\
\hline $\mathrm{N}-\mathrm{P}$ & 30342 & 1662 & 10963 & $42967(4.15 \%)$ \\
\hline VB-P & 23569 & 1308 & 8445 & $33322(3.22 \%)$ \\
\hline WPRO & 19677 & 1127 & 6926 & $27730(2.68 \%)$ \\
\hline ADV & 18360 & 1023 & 6490 & $25873(2.5 \%)$ \\
\hline $\mathrm{D}$ & 17841 & 953 & 6331 & $25125(2.43 \%)$ \\
\hline VB-D & 17515 & 937 & 6161 & $24613(2.38 \%)$ \\
\hline VB & 16426 & 932 & 5875 & $23233(2.24 \%)$ \\
\hline D-F & 15162 & 817 & 5272 & $21251(2.05 \%)$ \\
\hline $\mathrm{P}+\mathrm{D}$ & 13608 & 689 & 4964 & $19261(1.86 \%)$ \\
\hline C & 13214 & 737 & 4540 & $18491(1.79 \%)$ \\
\hline $\mathrm{P}+\mathrm{D}-\mathrm{F}$ & 12739 & 710 & 4492 & $17941(1.73 \%)$ \\
\hline $\mathrm{CL}$ & 11620 & 630 & 4063 & $16313(1.58 \%)$ \\
\hline NEG & 9656 & 563 & 3373 & $13592(1.31 \%)$ \\
\hline ADJ & 9035 & 494 & 3232 & $12761(1.23 \%)$ \\
\hline CONJS & 8112 & 464 & 2886 & $11462(1.11 \%)$ \\
\hline D-P & 7787 & 415 & 2672 & $10874(1.05 \%)$ \\
\hline ADJ-G & 7695 & 425 & 2649 & $10769(1.04 \%)$ \\
\hline ADV-R & 7492 & 464 & 2690 & $10646(1.03 \%)$ \\
\hline SE & 7577 & 445 & 2580 & $10602(1.02 \%)$ \\
\hline SR-P & 7487 & 407 & 2614 & $10508(1.01 \%)$ \\
\hline FW & 7679 & 390 & 2332 & $10401(1.0 \%)$ \\
\hline PRO\$-F & 7349 & 458 & 2530 & $10337(1.0 \%)$ \\
\hline ADJ-F & 6572 & 360 & 2228 & $9160(0.88 \%)$ \\
\hline PRO & 6354 & 360 & 2282 & $8996(0.87 \%)$ \\
\hline Q & 5608 & 325 & 1934 & $7867(0.76 \%)$ \\
\hline D-F-P & 5352 & 271 & 2043 & $7666(0.74 \%)$ \\
\hline $\mathrm{P}+\mathrm{D}-\mathrm{P}$ & 5325 & 313 & 1996 & $7634(0.74 \%)$ \\
\hline VB-SP & 4696 & 297 & 1543 & $6536(0.63 \%)$ \\
\hline NUM & 4487 & 212 & 1425 & $6124(0.59 \%)$ \\
\hline VB-AN & 4236 & 260 & 1486 & $5982(0.58 \%)$ \\
\hline D-UM & 3887 & 208 & 1395 & $5490(0.53 \%)$ \\
\hline $\mathrm{P}+\mathrm{D}-\mathrm{F}-\mathrm{P}$ & 3803 & 205 & 1348 & $5356(0.52 \%)$ \\
\hline VB-G & 3770 & 203 & 1295 & $5268(0.51 \%)$ \\
\hline PRO\$ & 3530 & 185 & 1292 & $5007(0.48 \%)$ \\
\hline ADJ-P & 3523 & 191 & 1183 & $4897(0.47 \%)$ \\
\hline( & 3405 & 199 & 1257 & $4861(0.47 \%)$ \\
\hline NPR-P & 3341 & 152 & 1204 & $4697(0.45 \%)$ \\
\hline Q-P & 2932 & 182 & 1017 & $4131(0.4 \%)$ \\
\hline SR-D & 2882 & 137 & 1039 & $4058(0.39 \%)$ \\
\hline ADJ-G-P & 2815 & 159 & 1030 & $4004(0.39 \%)$ \\
\hline VB-R & 2661 & 175 & 1038 & $3874(0.37 \%)$ \\
\hline D-UM-F & 2709 & 156 & 971 & $3836(0.37 \%)$ \\
\hline ADJ-F-P & 2510 & 133 & 935 & $3578(0.35 \%)$ \\
\hline TR-P & 2287 & 146 & 799 & $3232(0.31 \%)$ \\
\hline VB-AN-F & 2216 & 121 & 798 & $3135(0.3 \%)$ \\
\hline VB-SD & 2020 & 132 & 768 & $2920(0.28 \%)$ \\
\hline $\mathrm{P}+\mathrm{PRO}$ & 1968 & 110 & 732 & $2810(0.27 \%)$ \\
\hline VB-AN-P & 1836 & 107 & 694 & $2637(0.25 \%)$ \\
\hline Q-F-P & 1816 & 82 & 705 & $2603(0.25 \%)$ \\
\hline WADV & 1808 & 112 & 677 & $2597(0.25 \%)$ \\
\hline ADJ-R-G & 1788 & 85 & 618 & $2491(0.24 \%)$ \\
\hline $\mathrm{FP}$ & 1659 & 101 & 661 & $2421(0.23 \%)$ \\
\hline $\mathrm{Q}-\mathrm{F}$ & 1597 & 125 & 566 & $2288(0.22 \%)$ \\
\hline PRO\$-P & 1553 & 108 & 548 & $2209(0.21 \%)$ \\
\hline DEM & 1524 & 79 & 572 & $2175(0.21 \%)$ \\
\hline
\end{tabular}




\begin{tabular}{|c|c|c|c|}
\hline & Continu & ão da $T$ & ela A. \\
\hline Etiquetas & Treino & Desen. & Teste \\
\hline CONJ-NEG & 1482 & 75 & 564 \\
\hline SR & 1471 & 82 & 501 \\
\hline HV-P & 1450 & 85 & 508 \\
\hline VB-PP & 1363 & 86 & 500 \\
\hline QT & 1401 & 58 & 480 \\
\hline $\mathrm{VB}+\mathrm{CL}$ & 1317 & 82 & 468 \\
\hline TR-D & 1285 & 65 & 436 \\
\hline PRO\$-F-P & 1252 & 55 & 414 \\
\hline ET-P & 1158 & 81 & 397 \\
\hline VB-RA & 1147 & 59 & 390 \\
\hline VB-AN-F-P & 1090 & 65 & 397 \\
\hline VB-F & 1051 & 49 & 367 \\
\hline VB-SR & 869 & 56 & 305 \\
\hline Q-NEG & 804 & 42 & 325 \\
\hline OUTRO-P & 770 & 54 & 257 \\
\hline HV-D & 772 & 38 & 226 \\
\hline $\mathrm{Q}-\mathrm{G}$ & 725 & 38 & 252 \\
\hline OUTRO & 680 & 36 & 250 \\
\hline SR-R & 704 & 32 & 226 \\
\hline $\mathrm{VB}-\mathrm{G}+\mathrm{CL}$ & 681 & 31 & 233 \\
\hline OUTRO-F & 636 & 35 & 274 \\
\hline SR-SP & 671 & 32 & 225 \\
\hline ET-D & 635 & 31 & 223 \\
\hline $\mathrm{VB}+\mathrm{SE}$ & 628 & 33 & 210 \\
\hline VB-D+CL & 594 & 39 & 217 \\
\hline $\mathrm{HV}-\mathrm{P}+\mathrm{P}$ & 587 & 33 & 225 \\
\hline SENAO & 577 & 27 & 209 \\
\hline VB-P+SE & 572 & 35 & 197 \\
\hline $\mathrm{VB}-\mathrm{P}+\mathrm{CL}$ & 582 & 23 & 198 \\
\hline $\mathrm{TR}$ & 562 & 40 & 199 \\
\hline OUTRO-F-P & 554 & 28 & 183 \\
\hline VB-D+SE & 537 & 20 & 175 \\
\hline WD & 452 & 29 & 164 \\
\hline ADJ-R-G-P & 455 & 17 & 172 \\
\hline SR-G & 456 & 19 & 142 \\
\hline $\mathrm{P}+\mathrm{DEM}$ & 391 & 16 & 148 \\
\hline $\mathrm{VB}-\mathrm{G}+\mathrm{SE}$ & 354 & 27 & 148 \\
\hline ADJ-S & 369 & 23 & 115 \\
\hline ADV-NEG & 344 & 19 & 135 \\
\hline SR-SD & 319 & 19 & 128 \\
\hline NUM-F & 306 & 22 & 127 \\
\hline VB-I & 330 & 13 & 100 \\
\hline $\mathrm{P}+\mathrm{ADV}$ & 303 & 19 & 120 \\
\hline WQ & 330 & 13 & 95 \\
\hline $\mathrm{P}+\mathrm{WADV}$ & 306 & 11 & 103 \\
\hline $\mathrm{CL}+\mathrm{CL}$ & 279 & 11 & 100 \\
\hline HV & 265 & 16 & 102 \\
\hline WPRO-P & 259 & 19 & 102 \\
\hline D-UM-P & 266 & 12 & 91 \\
\hline ADJ-S-F & 246 & 31 & 86 \\
\hline INTJ & 248 & 14 & 88 \\
\hline Q-NEG-F & 222 & 12 & 75 \\
\hline TR-SP & 208 & 7 & 86 \\
\hline VB-SP+CL & 211 & 12 & 66 \\
\hline ADJ-R-F-P & 209 & 9 & 63 \\
\hline ADJ-R-F & 196 & 9 & 68 \\
\hline ET & 183 & 10 & 75 \\
\hline ADJ-R-P & 184 & 13 & 65 \\
\hline SR-RA & 191 & 3 & 56 \\
\hline SR-SR & 183 & 8 & 55 \\
\hline TR-R & 174 & 7 & 57 \\
\hline VB-SP+SE & 132 & 10 & 64 \\
\hline D-UM-F-P & 134 & 11 & 60 \\
\hline SR-F & 141 & 9 & 53 \\
\hline TR-G & 137 & 4 & 44 \\
\hline ADJ-R & 123 & 4 & 54 \\
\hline VB-I+CL & 127 & 6 & 44 \\
\hline WPRO\$-F & 112 & 8 & 47 \\
\hline WD-P & 119 & 8 & 36 \\
\hline HV-SP & 107 & 7 & 34 \\
\hline $\mathrm{P}+\mathrm{D}-\mathrm{UM}$ & 117 & 3 & 27 \\
\hline ADJ-S-P & 99 & 3 & 38 \\
\hline TR-SD & 94 & 8 & 35 \\
\hline $\mathrm{HV}-\mathrm{G}$ & 93 & 1 & 30 \\
\hline $\mathrm{P}+\mathrm{D}-\mathrm{UM}-\mathrm{F}$ & 78 & 8 & 36 \\
\hline ET-G & 87 & 5 & 29 \\
\hline WPRO\$ & 87 & 3 & 28 \\
\hline $\mathrm{P}+\mathrm{NPR}$ & 85 & 5 & 28 \\
\hline HV-SD & 77 & 4 & 33 \\
\hline HV-R & 83 & 2 & 20 \\
\hline ADJ-S-F-P & 57 & 4 & 24 \\
\hline SR-PP & 59 & 2 & 24 \\
\hline $\mathrm{D}-\mathrm{G}$ & 61 & 3 & 17 \\
\hline TR-RA & 52 & 3 & 20 \\
\hline VB-R!CL & 54 & 1 & 18 \\
\hline ET-R & 54 & 1 & 16 \\
\hline ET-SP & 50 & 3 & 18 \\
\hline TR-F & 52 & 0 & 18 \\
\hline TR-SR & 47 & 3 & 20 \\
\hline HV-RA & 40 & 4 & 19 \\
\hline WPRO\$-P & 39 & 1 & 20 \\
\hline HV-SR & 42 & 2 & 10 \\
\hline WPRO $\$-F-P$ & 31 & 3 & 16 \\
\hline
\end{tabular}




\begin{tabular}{|c|c|c|c|c|}
\hline \multicolumn{5}{|c|}{ Continuação da Tabela A.1 } \\
\hline Etiquetas & Treino & Desen. & Teste & Total \\
\hline WD-F-P & 34 & 2 & 11 & $47(0.0 \%)$ \\
\hline $\mathrm{VB}-\mathrm{F}+\mathrm{SE}$ & 33 & 1 & 12 & $46(0.0 \%)$ \\
\hline $\mathrm{P}+\mathrm{WPRO}$ & 31 & 3 & 12 & $46(0.0 \%)$ \\
\hline $\mathrm{P}+\mathrm{CL}$ & 31 & 0 & 14 & $45(0.0 \%)$ \\
\hline $\mathrm{VB}-\mathrm{F}+\mathrm{CL}$ & 30 & 1 & 11 & $42(0.0 \%)$ \\
\hline D-G-P & 28 & 2 & 6 & $36(0.0 \%)$ \\
\hline $\mathrm{XX}$ & 28 & 2 & 5 & $35(0.0 \%)$ \\
\hline $\mathrm{VB}-\mathrm{P}+\mathrm{SE}+\mathrm{CL}$ & 27 & 0 & 8 & $35(0.0 \%)$ \\
\hline $\mathrm{VB}+\mathrm{CL}+\mathrm{CL}$ & 20 & 1 & 14 & $35(0.0 \%)$ \\
\hline ET-SR & 25 & 1 & 6 & $32(0.0 \%)$ \\
\hline VB-R!SE & 18 & 2 & 10 & $30(0.0 \%)$ \\
\hline ET-RA & 24 & 0 & 5 & $29(0.0 \%)$ \\
\hline $\mathrm{P}+$ OUTRO-F & 20 & 2 & 7 & $29(0.0 \%)$ \\
\hline TR-PP & 20 & 1 & 7 & $28(0.0 \%)$ \\
\hline ADV-S & 20 & 2 & 5 & $27(0.0 \%)$ \\
\hline ET-SD & 21 & 0 & 6 & $27(0.0 \%)$ \\
\hline $\mathrm{HV}-\mathrm{F}$ & 17 & 0 & 10 & $27(0.0 \%)$ \\
\hline ET-F & 14 & 0 & 11 & $25(0.0 \%)$ \\
\hline Q-NEG-P & 11 & 1 & 13 & $25(0.0 \%)$ \\
\hline $\mathrm{P}+$ OUTRO-F-P & 16 & 2 & 6 & $24(0.0 \%)$ \\
\hline VB-G+SE+CL & 18 & 0 & 5 & $23(0.0 \%)$ \\
\hline WD-F & 16 & 0 & 5 & $21(0.0 \%)$ \\
\hline VB-D+SE+CL & 14 & 0 & 5 & $19(0.0 \%)$ \\
\hline TR-P+CL & 13 & 0 & 6 & $19(0.0 \%)$ \\
\hline $\mathrm{HV}-\mathrm{G}+\mathrm{SE}$ & 13 & 1 & 5 & $19(0.0 \%)$ \\
\hline WPRO-F-P & 12 & 2 & 4 & $18(0.0 \%)$ \\
\hline $\mathrm{HV}-\mathrm{PP}$ & 11 & 2 & 5 & $18(0.0 \%)$ \\
\hline ET-PP & 10 & 1 & 6 & $17(0.0 \%)$ \\
\hline P+OUTRO & 12 & 1 & 4 & $17(0.0 \%)$ \\
\hline $\mathrm{P}+$ OUTRO-P & 7 & 3 & 5 & $15(0.0 \%)$ \\
\hline Q-G-P & 11 & 0 & 4 & $15(0.0 \%)$ \\
\hline $\mathrm{TR}+\mathrm{CL}$ & 10 & 1 & 3 & $14(0.0 \%)$ \\
\hline TR-G+CL & 12 & 0 & 1 & $13(0.0 \%)$ \\
\hline $\mathrm{HV}+\mathrm{CL}$ & 9 & 0 & 2 & $11(0.0 \%)$ \\
\hline Q-NEG-F-P & 8 & 2 & 1 & $11(0.0 \%)$ \\
\hline TR-D+CL & 8 & 2 & 1 & $11(0.0 \%)$ \\
\hline TR-I & 6 & 1 & 4 & $11(0.0 \%)$ \\
\hline $\mathrm{SR}+\mathrm{CL}$ & 7 & 1 & 2 & $10(0.0 \%)$ \\
\hline VB-RA+CL & 7 & 0 & 3 & $10(0.0 \%)$ \\
\hline $\mathrm{VB}+\mathrm{SE}+\mathrm{CL}$ & 7 & 1 & 2 & $10(0.0 \%)$ \\
\hline VB-RA+SE & 5 & 0 & 5 & $10(0.0 \%)$ \\
\hline $\mathrm{HV}-\mathrm{G}+\mathrm{CL}$ & 8 & 0 & 1 & $9(0.0 \%)$ \\
\hline ET-I & 7 & 0 & 2 & $9(0.0 \%)$ \\
\hline TR-SP+CL & 6 & 0 & 2 & $8(0.0 \%)$ \\
\hline $\mathrm{HV}-\mathrm{P}+\mathrm{CL}$ & 6 & 0 & 2 & $8(0.0 \%)$ \\
\hline SR-P+CL & 6 & 1 & 1 & $8(0.0 \%)$ \\
\hline SR-G+CL & 6 & 1 & 1 & $8(0.0 \%)$ \\
\hline$P+Q-F$ & 3 & 2 & 2 & $7(0.0 \%)$ \\
\hline TR-AN & 1 & 0 & 6 & $7(0.0 \%)$ \\
\hline $\mathrm{P}+\mathrm{D}-\mathrm{UM}-\mathrm{P}$ & 3 & 1 & 2 & $6(0.0 \%)$ \\
\hline $\mathrm{P}+\mathrm{Q}$ & 5 & 0 & 1 & $6(0.0 \%)$ \\
\hline $\mathrm{P}+\mathrm{Q}-\mathrm{P}$ & 6 & 0 & 0 & $6(0.0 \%)$ \\
\hline WPRO-F & 5 & 0 & 1 & $6(0.0 \%)$ \\
\hline SR-D+CL & 5 & 0 & 1 & $6(0.0 \%)$ \\
\hline $\mathrm{P}+\mathrm{Q}-\mathrm{F}-\mathrm{P}$ & 5 & 0 & 1 & $6(0.0 \%)$ \\
\hline $\mathrm{ET}-\mathrm{P}+\mathrm{CL}$ & 5 & 0 & 1 & $6(0.0 \%)$ \\
\hline VB-P+P & 5 & 0 & 1 & $6(0.0 \%)$ \\
\hline $\mathrm{ADV}+\mathrm{CL}$ & 4 & 0 & 1 & $5(0.0 \%)$ \\
\hline $\mathrm{HV}-\mathrm{AN}-\mathrm{P}$ & 3 & 0 & 2 & $5(0.0 \%)$ \\
\hline $\mathrm{VB}-\mathrm{SP}+\mathrm{SE}+\mathrm{CL}$ & 3 & 0 & 1 & $4(0.0 \%)$ \\
\hline $\mathrm{HV}+\mathrm{SE}$ & 2 & 0 & 2 & $4(0.0 \%)$ \\
\hline TR-G+SE & 2 & 1 & 1 & $4(0.0 \%)$ \\
\hline $\mathrm{TR}+\mathrm{SE}$ & 2 & 0 & 2 & $4(0.0 \%)$ \\
\hline TR-P+SE & 2 & 1 & 1 & $4(0.0 \%)$ \\
\hline SR-I & 0 & 0 & 4 & $4(0.0 \%)$ \\
\hline $\mathrm{VB}-\mathrm{SP}+\mathrm{CL}+\mathrm{CL}$ & 4 & 0 & 0 & $4(0.0 \%)$ \\
\hline HV-I & 3 & 0 & 1 & $4(0.0 \%)$ \\
\hline $\mathrm{P}+\mathrm{WD}$ & 4 & 0 & 0 & $4(0.0 \%)$ \\
\hline $\mathrm{VB}-\mathrm{G}+\mathrm{CL}+\mathrm{CL}$ & 4 & 0 & 0 & $4(0.0 \%)$ \\
\hline VB-SR+CL & 4 & 0 & 0 & $4(0.0 \%)$ \\
\hline $\mathrm{P}+\mathrm{D}-\mathrm{UM}-\mathrm{F}-\mathrm{P}$ & 4 & 0 & 0 & $4(0.0 \%)$ \\
\hline TR-I+CL & 1 & 1 & 1 & $3(0.0 \%)$ \\
\hline $\mathrm{VB}-\mathrm{D}+\mathrm{CL}+\mathrm{CL}$ & 3 & 0 & 0 & $3(0.0 \%)$ \\
\hline HV-AN-F & 1 & 0 & 2 & $3(0.0 \%)$ \\
\hline ET-P+SE & 3 & 0 & 0 & $3(0.0 \%)$ \\
\hline VB-SR+SE & 3 & 0 & 0 & $3(0.0 \%)$ \\
\hline D+OUTRO & 1 & 0 & 2 & $3(0.0 \%)$ \\
\hline TR-F+CL & 2 & 0 & 1 & $3(0.0 \%)$ \\
\hline $\mathrm{VB}-\mathrm{P}+\mathrm{CL}+\mathrm{CL}$ & 1 & 0 & 1 & $2(0.0 \%)$ \\
\hline $\mathrm{VB}-\mathrm{F}+\mathrm{CL}+\mathrm{CL}$ & 1 & 0 & 1 & $2(0.0 \%)$ \\
\hline $\mathrm{HV}-\mathrm{F}+\mathrm{SE}$ & 1 & 0 & 1 & $2(0.0 \%)$ \\
\hline VB-SD+CL & 1 & 0 & 1 & $2(0.0 \%)$ \\
\hline SR-I+CL & 1 & 0 & 1 & $2(0.0 \%)$ \\
\hline TR-D+SE & 1 & 0 & 1 & $2(0.0 \%)$ \\
\hline SR-R!CL & 1 & 0 & 1 & $2(0.0 \%)$ \\
\hline $\mathrm{HV}-\mathrm{P}+\mathrm{P}+\mathrm{SE}$ & 2 & 0 & 0 & $2(0.0 \%)$ \\
\hline $\mathrm{VB}+\mathrm{P}$ & 2 & 0 & 0 & $2(0.0 \%)$ \\
\hline
\end{tabular}




\begin{tabular}{lllll}
\hline \multicolumn{5}{c}{ Continuação da Tabela A.1 } \\
\hline Etiquetas & Treino & Desen. & Teste & Total \\
\hline HV-P+P+CL & 2 & 0 & 0 & $2(0.0 \%)$ \\
HV-P+SE & 2 & 0 & 0 & $2(0.0 \%)$ \\
VB-R+CL & 2 & 0 & 0 & $2(0.0 \%)$ \\
SR-SP+CL & 1 & 0 & 1 & $2(0.0 \%)$ \\
D+OUTRO-F & 2 & 0 & 0 & $2(0.0 \%)$ \\
HV-F+CL & 2 & 0 & 0 & $2(0.0 \%)$ \\
D+OUTRO-P & 2 & 0 & 0 & $2(0.0 \%)$ \\
TR-R!CL & 1 & 0 & 1 & $2(0.0 \%)$ \\
VB-I+CL+CL & 2 & 0 & 0 & $2(0.0 \%)$ \\
HV-G+CL+CL & 0 & 0 & 1 & $1(0.0 \%)$ \\
HV-SP+SE & 1 & 0 & 0 & $1(0.0 \%)$ \\
W & 1 & 0 & 0 & $1(0.0 \%)$ \\
TR+SE+CL & 1 & 0 & 0 & $1(0.0 \%)$ \\
HV-P+SE+CL & 1 & 0 & 0 & $1(0.0 \%)$ \\
D+OUTRO-F-P & 1 & 0 & 0 & $1(0.0 \%)$ \\
HV-AN-F-P & 1 & 0 & 0 & $1(0.0 \%)$ \\
TR-AN-P & 1 & 0 & 0 & $1(0.0 \%)$ \\
ET+CL & 1 & 0 & 0 & $1(0.0 \%)$ \\
SR-RA+CL & 1 & 0 & 0 & $1(0.0 \%)$ \\
ET-D+CL & 1 & 0 & 0 & $1(0.0 \%)$ \\
ET-D+SE & 1 & 0 & 0 & $1(0.0 \%)$ \\
HV-RA+SE+CL & 1 & 0 & 0 & $1(0.0 \%)$ \\
SR-F+CL & 1 & 0 & 0 & $1(0.0 \%)$ \\
HV-AN & 0 & 0 & 1 & $1(0.0 \%)$ \\
\hline
\end{tabular}

Na Tabela A.2 temos a descrição das 265 etiquetas presentes no corpus, é possível encontra uma descrição completa em: http://www . tycho.iel. unicamp.br/corpus/manual/pos2016.html. 
Tabela A.2: Descrição e exemplos das etiquetas do corpus Tycho Brahe

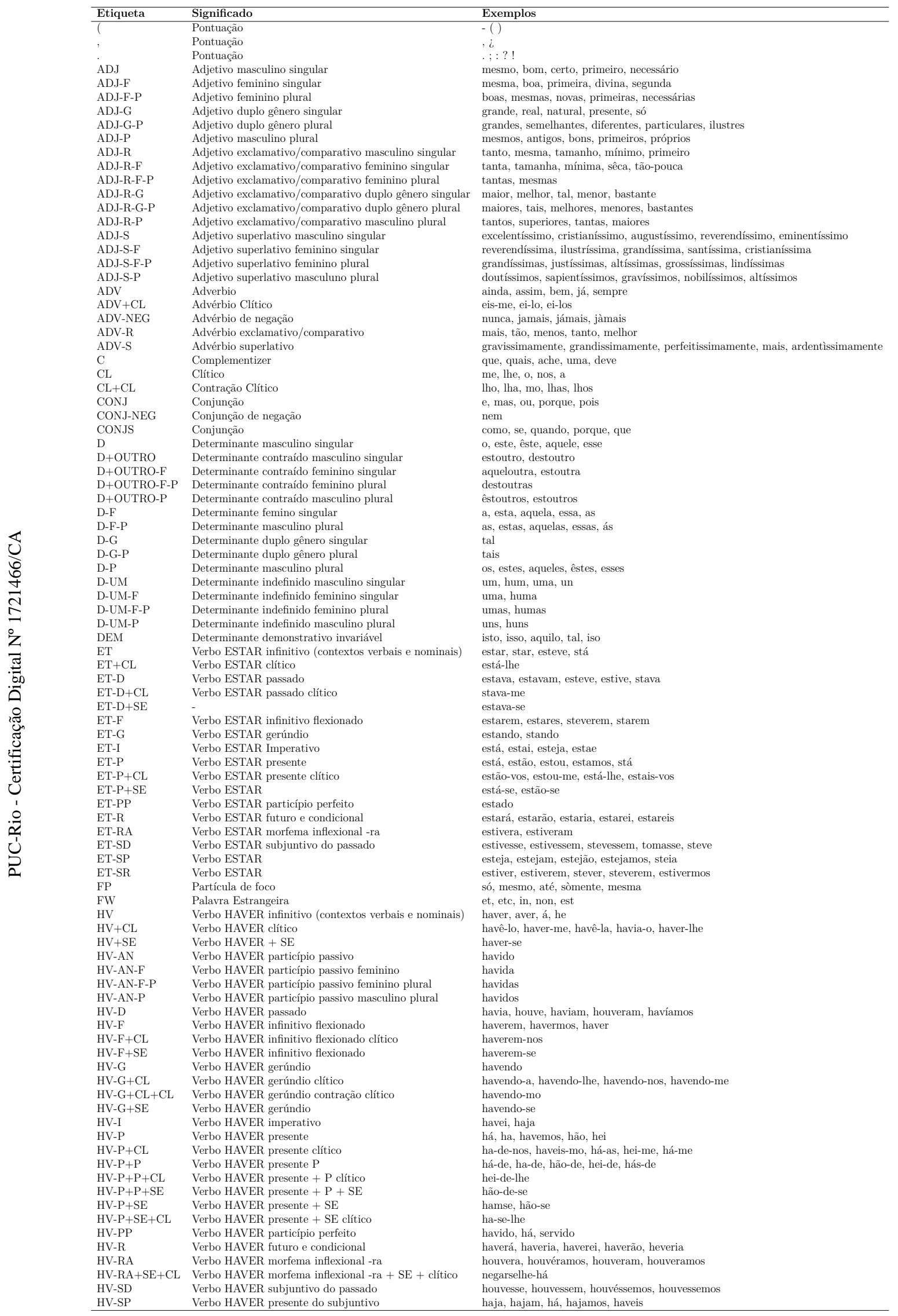




\begin{tabular}{|c|c|c|}
\hline \multicolumn{3}{|c|}{ Continuação da Tabela A.2 } \\
\hline Etiqueta & Significado & Exemplos \\
\hline $\mathrm{HV}-\mathrm{SP}+\mathrm{SE}$ & Verbo HAVER presente do subjuntivo + SE & hajam-se \\
\hline HV-SR & Verbo HAVER subjuntivo Futuro & houver, houverem \\
\hline INTJ & Interjeção & oh, ó, adeus, ah, ai \\
\hline $\mathrm{N}$ & Substantivo singular & tempo, parte, mundo, vida, dia \\
\hline $\mathrm{N}-\mathrm{P}$ & Substantivo plural & homens, vezes, anos, cousas, dias \\
\hline NEG & Negação & não, nem, nam, pois, nao \\
\hline NPR & Substantivo proprio singular & deus, mercê, senhor, excelência, dom \\
\hline NPR-P & Substantivo proprio plural & reis, portugueses, padres, índios, príncipes \\
\hline NUM & Número cardeal geral & mil, dois, três, quatro, dous \\
\hline NUM-F & Número & duas, quinhentas, duzentas, quatrocentas, oitocentas \\
\hline OUTRO & Tag OUTRO masculino & outro, reverendíssima, ilustríssima, outros, liso \\
\hline OUTRO-F & Tag OUTRO feminino & outra, outras \\
\hline OUTRO-F-P & Tag OUTRO feminino plural & outras, outra, essoutras \\
\hline OUTRO-P & Tag OUTRO masculino plural & outros, *outros, outro, estoutros \\
\hline $\mathrm{P}$ & Preposição & de, a, em, com, por \\
\hline $\mathrm{P}+\mathrm{ADV}$ & Preposição contração + ADV & daqui, dali, daí, d'aqui, d'ali \\
\hline $\mathrm{P}+\mathrm{CL}$ & Preposição contração + CL & pelo, pelos, polo, pelas, pela \\
\hline $\mathrm{P}+\mathrm{D}$ & Preposição contração + D & do, no, ao, pelo, neste \\
\hline $\mathrm{P}+\mathrm{D}-\mathrm{F}$ & Preposição contração + D-F & da, na, à, pela, desta \\
\hline $\mathrm{P}+\mathrm{D}-\mathrm{F}-\mathrm{P}$ & Preposição contração + D-F & das, nas, às, pelas, destas \\
\hline $\mathrm{P}+\mathrm{D}-\mathrm{P}$ & Preposição contração + D-P & dos, aos, nos, pelos, destes \\
\hline $\mathrm{P}+\mathrm{D}-\mathrm{UM}$ & Preposição contração + D-UM & dum, num, d'um, n'um, numa \\
\hline $\mathrm{P}+\mathrm{D}-\mathrm{UM}-\mathrm{F}$ & Preposição contração + D-UM-F & duma, numa, d'uma, n'uma \\
\hline $\mathrm{P}+\mathrm{D}-\mathrm{UM}-\mathrm{F}-\mathrm{P}$ & Preposição contração + D-UM-F-P & dumas \\
\hline $\mathrm{P}+\mathrm{D}-\mathrm{UM}-\mathrm{P}$ & Preposição contração + D-UM-P & duns, n'uns, nuns \\
\hline $\mathrm{P}+\mathrm{DEM}$ & Preposição contração + DEM & nisto, disto, disso, nisso, porisso \\
\hline $\mathrm{P}+\mathrm{NPR}$ & Preposição contração + NPR & d'elrei, delrei, d'el-rei, del-rei, delrey \\
\hline $\mathrm{P}+\mathrm{OUTRO}$ & Preposição contração + OUTRO & noutro, doutro, d'outro, n'outro \\
\hline P+OUTRO-F & Preposição contração + OUTRO-F & noutra, d'outra, doutra, n'outra \\
\hline $\mathrm{P}+\mathrm{OUTRO}-\mathrm{F}-\mathrm{P}$ & Preposição contração + OUTRO-F-P & d'outras, noutras, n'outras, doutras, noutra \\
\hline $\mathrm{P}+\mathrm{OUTRO}-\mathrm{P}$ & Preposição contração + OUTRO-P & noutros, d'outros, doutros, doutos, n'outros \\
\hline $\mathrm{P}+\mathrm{PRO}$ & Preposição contração + PRO & dela, dele, nela, delas, deles \\
\hline $\mathrm{P}+\mathrm{Q}$ & Preposição contração + Q & d'algum, d'outrem, dalgum, doutrém, nalgum \\
\hline $\mathrm{P}+\mathrm{Q}-\mathrm{F}$ & Preposição contração + Q-F & d'alguma, n'alguma, dalguma \\
\hline $\mathrm{P}+\mathrm{Q}-\mathrm{F}-\mathrm{P}$ & Preposição contração + Q-F-P & d'algumas, entr'ambas, dalgumas \\
\hline $\mathrm{P}+\mathrm{Q}-\mathrm{P}$ & Preposição contração + Q-P & d'alguns, d'ambos \\
\hline $\mathrm{P}+\mathrm{WADV}$ & Preposição contração + WADV & donde, aonde, d'onde, adonde, daqui \\
\hline $\mathrm{P}+\mathrm{WD}$ & Preposição contração + WD & porque \\
\hline $\mathrm{P}+\mathrm{WPRO}$ & Preposição contração + WPRO & porque, porquê \\
\hline $\mathrm{PRO}$ & Pronome oblíquo/pessoal & eu, ele, êle, si, ela \\
\hline PRO\$ & Pronome masculino singular & seu, meu, nosso, vosso, teu \\
\hline PRO\$-F & Pronome singular feminino & vossa, sua, minha, nossa, tua \\
\hline PRO\$-F-P & Pronome plural feminino & suas, minhas, nossas, vossas, tuas \\
\hline PRO\$-P & Pronome masculino plural & seus, nossos, meus, vossos, teus \\
\hline Q & Quantificador & muito, tudo, todo, pouco, algum \\
\hline $\mathrm{Q}-\mathrm{F}$ & Quantificador feminino & alguma, toda, muita, tôda, pouca \\
\hline Q-F-P & Quantificador feminino plural & todas, muitas, algumas, tôdas, poucas \\
\hline Q-G & Quantificador duplo gênero singular & cada, qualquer, algum \\
\hline Q-G-P & Quantificador duplo gênero plural & quaisquer, quaesquer \\
\hline Q-NEG & Quantificador de negação & nada, nenhum, ninguém, ninguem, alguém \\
\hline Q-NEG-F & Quantificador de negação feminino & nenhuma \\
\hline Q-NEG-F-P & Quantificador de negação feminino plural & nenhumas \\
\hline Q-NEG-P & Quantificador de negação plural & alguns, nenhuns \\
\hline $\mathrm{Q}-\mathrm{P}$ & Quantificador Plural & todos, muitos, alguns, poucos, ambos \\
\hline QT & Aspa & " \\
\hline SE & $\mathrm{Se}$ & se \\
\hline SENAO & Senão & senão, senã \\
\hline $\mathrm{SR}$ & Verbo SER infinitivo (contextos verbais e nominais) & ser, é, ri, haverá, desejo \\
\hline $\mathrm{SR}+\mathrm{CL}$ & Verbo SER clítico & sê-lo, ser-lhes, ser-lhe, ser-me, ser-vos \\
\hline SR-D & Verbo SER passado & era, foi, eram, foram, fui \\
\hline SR-D+CL & Verbo SER passado clítico & foi-lhe, era-me, foi-me, foi-lhes \\
\hline SR-F & Verbo SER futuro & serem, serdes, sermos, seres, ser \\
\hline $\mathrm{SR}-\mathrm{F}+\mathrm{CL}$ & Verbo SER futuro clítico & serem-lhe \\
\hline SR-G & Verbo SER gerúndio & sendo \\
\hline $\mathrm{SR}-\mathrm{G}+\mathrm{CL}$ & Verbo SER gerúndio clítico & sendo-lhe, sendo-me \\
\hline SR-I & Verbo SER imperativo & sede, sê \\
\hline $\mathrm{SR}-\mathrm{I}+\mathrm{CL}$ & Verbo SER imperativo clítico & achai-vos, sêde-lhes \\
\hline SR-P & Verbo SER presente & é, são, sou, sois, somos \\
\hline $\mathrm{SR}-\mathrm{P}+\mathrm{CL}$ & Verbo SER presente clítico & é-nos, são-me, é-me, é-lhes, são-lhe \\
\hline SR-PP & Verbo SER particípio perfeito & sido \\
\hline SR-R & Verbo SER futuro e condicional & será, seria, serão, haverá, seriam \\
\hline SR-R!CL & Verbo SER mesóclise & ser-me-ia, serlhes-ha \\
\hline SR-RA & Verbo SER morfema inflexional -ra & fôra, fora, foram, fôras, fôreis \\
\hline SR-RA+CL & Verbo SER morfema inflexional -ra clítico & fôra-o \\
\hline SR-SD & Verbo SER subjuntivo do passado & fosse, fôsse, fossem, fôssem, fôsseis \\
\hline SR-SP & Verbo SER presente do subjuntivo & seja, sejam, sejais, sejão, sejas \\
\hline $\mathrm{SR}-\mathrm{SP}+\mathrm{CL}$ & Verbo SER presente do subjuntivo clítico & seja-me \\
\hline SR-SR & Verbo SER subjuntivo futuro & for, fôr, forem, fôrem, formos \\
\hline
\end{tabular}




\begin{tabular}{|c|c|c|}
\hline & Contint & ação da Tabela A.2 \\
\hline Etiqueta & Significado & Exemplos \\
\hline TR & Verbo TER infinitivo (contextos verbais e nominais) & ter, têem, ternas, desejo, posso \\
\hline $\mathrm{TR}+\mathrm{CL}$ & Verbo TER clítico & tê-la, ter-nos, tê-lo, ter-lhe, ter-la \\
\hline $\mathrm{TR}+\mathrm{SE}$ & Verbo TER + SE & ter-se, terminar-se \\
\hline $\mathrm{TR}+\mathrm{SE}+\mathrm{CL}$ & Verbo TER + SE clítico & ter-se-lhe \\
\hline TR-AN & Verbo TER particípio passivo & tido \\
\hline TR-AN-P & Verbo TER particípio passivo presente & tidos \\
\hline TR-D & Verbo TER passado & tinha, teve, tinham, tive, tiveram \\
\hline TR-D+CL & Verbo TER passado clítico & tinha-me, tinha-o, tinha-lhe, tinhalhe, tinhão-o \\
\hline TR-D+SE & Verbo TER passado + SE & tinha-se \\
\hline TR-F & Verbo TER futuro & terem, termos, terdes, téremos \\
\hline $\mathrm{TR}-\mathrm{F}+\mathrm{CL}$ & Verbo TER futuro clítico & terem-no, terem-nos, terem-na \\
\hline TR-G & Verbo TER gerúndio & tendo \\
\hline $\mathrm{TR}-\mathrm{G}+\mathrm{CL}$ & Verbo TER gerúndio clítico & tendo-o, tendo-me, tendo-vos, tergiversandolhe, tendo-os \\
\hline TR-G+SE & Verbo TER gerúndio + SE & tendo-se \\
\hline TR-I & Verbo TER imperativo & tende, tem \\
\hline TR-I+CL & Verbo TER imperativo clítico & tende-o \\
\hline TR-P & Verbo TER presente & tem, tenho, têm, temos, tendes \\
\hline $\mathrm{TR}-\mathrm{P}+\mathrm{CL}$ & Verbo TER presente clítico & tenho-o, tem-me, tem-na, tenho-me, tem-no \\
\hline TR-P+SE & Verbo TER presente + SE & tem-se, têm-se, têem-se \\
\hline TR-PP & Verbo TER particípio perfeito & tido \\
\hline TR-R & Verbo TER futuro & terá, teria, terei, terão, teriam \\
\hline TR-R!CL & Verbo TER futuro mesóclise & telo-hão, tel-o-hão \\
\hline TR-RA & Verbo TER morfema inflexional -ra & tivera, tevera, tiveram, tevéramos, tiveras \\
\hline TR-SD & Verbo TER subjuntivo do passado & tivesse, tivessem, tivéssemos, tevessemos, teverem \\
\hline TR-SP & Verbo TER presente do subjuntivo & tenha, tenham, tenhamos, tenhais, tenhão \\
\hline TR-SP+CL & Verbo TER presente do subjuntivo & tenha-me, tenha-o, tenha-lhe \\
\hline TR-SR & Verbo TER subjuntivo futuro & tiver, tiverem, tever, tivermos, tiverdes \\
\hline VB & Verbo & fazer, dizer, dar, ver, ir \\
\hline $\mathrm{VB}+\mathrm{CL}$ & Verbo clítico & dar-lhe, fazê-lo, dizer-lhe, servi-lo, dar-me \\
\hline $\mathrm{VB}+\mathrm{CL}+\mathrm{CL}$ & Verbo contração clítico & dar-lhas, dizer-lho, querer-lh'a, insinuar-lho, fazer-no-lo \\
\hline $\mathrm{VB}+\mathrm{P}$ & Verbo $+\mathrm{P}$ & hás-de \\
\hline $\mathrm{VB}+\mathrm{SE}$ & Verbo $+\mathrm{SE}$ & fazer-se, servir-se, ver-se, lembrar-se, conservar-se \\
\hline $\mathrm{VB}+\mathrm{SE}+\mathrm{CL}$ & Verbo + SE clítico & cortar-se-lhe, conferir-se-lhe, oferecem-se-lhe, dar-se-lhe, comunicar-se-nos \\
\hline VB-AN & Verbo particípio passivo masculino singular & posto, obrigado, passado, visto, dito \\
\hline VB-AN-F & Verbo particípio passivo feminino singular & feita, dita, querida, passada, conhecida \\
\hline VB-AN-F-P & Verbo particípio passivo feminino plural & feitas, escritas, ditas, passadas, pintadas \\
\hline VB-AN-P & Verbo particípio passivo masculino plural & passados, mortos, obrigados, ditos, feitos \\
\hline VB-D & Verbo passado & disse, fez, podia, mandou, deu \\
\hline VB-D+CL & Verbo passado clítico & disse-lhe, disse-me, deu-lhe, pareceu-me, mandou-lhe \\
\hline $\mathrm{VB}-\mathrm{D}+\mathrm{CL}+\mathrm{CL}$ & Verbo passado contração clítico & acendeu-lho, tornou-mo, quebrou-lhos \\
\hline VB-D+SE & Verbo passado $+\mathrm{SE}$ & foi-se, seguiu-se, viu-se, fez-se, pôs-se \\
\hline $\mathrm{VB}-\mathrm{D}+\mathrm{SE}+\mathrm{CL}$ & Verbo passado + SE clítico & ofereceu-se-lhe, foi-se-lhe, pegou-se-me, quebrou-se-lhe, meteu-se-lhe \\
\hline VB-F & Verbo infinitivo Infletido & fazerem, darem, poderem, irem, virem \\
\hline $\mathrm{VB}-\mathrm{F}+\mathrm{CL}$ & Verbo infinitivo Infletido clítico & darem-lhe, darem-me, estimarem-nos, preguntarem-me, recusarem-lhe \\
\hline $\mathrm{VB}-\mathrm{F}+\mathrm{CL}+\mathrm{CL}$ & Verbo infinitivo Infletido clítico contração clítico & dardes-mas, darmos-lhas \\
\hline VB-F+SE & Verbo infinitivo Infletido + SE & irem-se, darem-se, acharem-se, deixarem-se, verem-se \\
\hline VB-G & Verbo gerúndio & fazendo, vendo, dizendo, dando, deixando \\
\hline VB-G+CL & Verbo gerúndio clítico & dando-lhe, dizendo-lhe, pedindo-lhe, fazendo-lhe, mandando-lhe \\
\hline $\mathrm{VB}-\mathrm{G}+\mathrm{CL}+\mathrm{CL}$ & Verbo gerúndio contração clítico & permittindo-lho, dando-lha, quadruplicandolho, mandando-ma \\
\hline VB-G+SE & Verbo gerúndio + SE & vendo-se, fazendo-se, achando-se, metendo-se, dando-se \\
\hline $\mathrm{VB}-\mathrm{G}+\mathrm{SE}+\mathrm{CL}$ & Verbo gerúndio + SE clítico & dando-se-lhe, oferecendo-se-lhe, pagando-se-lhe, oferecendose-lhe, preguntando-se-lhe \\
\hline VB-I & Verbo Imperativo & vêde, fazei, sabei, dá, vinde \\
\hline VB-I+CL & Verbo Imperativo clítico & perdoai-me, dai-me, dizei-me, lembrai-vos, dizei-lhe \\
\hline $\mathrm{VB}-\mathrm{I}+\mathrm{CL}+\mathrm{CL}$ & Verbo Imperativo contração clítico & guardai-ma, mostrai-lho \\
\hline VB-P & Verbo presente & pode, faz, parece, deve, diz \\
\hline $\mathrm{VB}-\mathrm{P}+\mathrm{CL}$ & Verbo presente clítico & parece-me, parece-nos, julgam-vos, lembra-me, digo-vos \\
\hline $\mathrm{VB}-\mathrm{P}+\mathrm{CL}+\mathrm{CL}$ & Verbo presente contração clítico & pedem-no-la, digo-vo-lo \\
\hline $\mathrm{VB}-\mathrm{P}+\mathrm{P}$ & Verbo presente $+\mathrm{P}$ & hás-de, dá-de \\
\hline VB-P+SE & Verbo presente $+\mathrm{SE}$ & deve-se, pode-se, segue-se, acha-se, devem-se \\
\hline $\mathrm{VB}-\mathrm{P}+\mathrm{SE}+\mathrm{CL}$ & Verbo presente + SE clítico & dá-se-lhe, deita-se-lhe, suspendeu-se-lhe, segue-se-lhe, regula-se-lhe \\
\hline VB-PP & Verbo particípio perfeito & feito, dito, dado, visto, passado \\
\hline VB-R & Verbo futuro & poderá, fará, poderia, direi, verá \\
\hline VB-R!CL & Verbo futuro mesóclise & chamar-me-eis, obrigá-los-ia, dar-lhe-ia, dir-me-ão, dir-me-á \\
\hline VB-R!SE & Verbo futuro + SE & salvar-se-ha, farse-ha, continuar-se-ia, enfadar-se-ía, livrarse-ha \\
\hline $\mathrm{VB}-\mathrm{R}+\mathrm{CL}$ & Verbo futuro clítico & mandá-la, passá-los \\
\hline VB-RA & Verbo morfema inflexional -ra & pudera, quisera, fizera, tomara, dera \\
\hline VB-RA+CL & Verbo morfema inflexional -ra clítico & apresentara-me, quisera-me, atrevera-me, tomarame, estimara-o \\
\hline VB-RA+SE & Verbo morfema inflexional -ra + SE & pudera-se, implicara-se, familiarizara-se, podera-se, perdera-se \\
\hline VB-SD & Verbo subjuntivo do passado & fizesse, pudesse, desse, quisesse, fossem \\
\hline $\mathrm{VB}-\mathrm{SD}+\mathrm{CL}$ & Verbo subjuntivo do passado clítico & esqueceu-me, condemnassem-no \\
\hline VB-SP & Verbo presente do subjuntivo & guarde, faça, possa, queira, dê \\
\hline VB-SP+CL & Verbo presente do subjuntivo clítico & dê-me, mande-me, encomende-me, diga-me, diga-lhe \\
\hline VB-SP+CL+CL & Verbo presente do subjuntivo contração clítico & lance-ma, mande-mas, dê-vo-la, perdoe-mo \\
\hline VB-SP+SE & Verbo presente do subjuntivo $+\mathrm{SE}$ & lembre-se, sirva-se, faça-se, contente-se, veja-se \\
\hline $\mathrm{VB}-\mathrm{SP}+\mathrm{SE}+\mathrm{CL}$ & Verbo presente do subjuntivo + SE clítico & satisfaçam-se-me, deva-se-lhes, contem-selhe, ponha-se-me \\
\hline VB-SR & Verbo subjuntivo futuro & quiser, puder, der, fizer, disser \\
\hline VB-SR+CL & Verbo subjuntivo futuro clítico & fazerem-me, serem-lhe, saberdes-vos \\
\hline VB-SR+SE & Verbo subjuntivo futuro + SE & pintarem-se, passarem-se, verem-se \\
\hline W & nido & nido \\
\hline WADV & Elemento interrogativo & onde, quanto, como, porque, quão \\
\hline WD & Determinante interrogativo masculino & que, qual, quanto, se, quê \\
\hline WD-F & Determinante interrogativo feminino & quanta, quais, cuja \\
\hline WD-F-P & Determinante interrogativo feminino plural & quantas \\
\hline WD-P & Determinante interrogativo masculino plural & quais, quantos, quantas, que, quaes \\
\hline WPRO & Elemento relativo & que, quem, qual, quanto, quê \\
\hline WPRO\$ & Elemento relativo CUJO masculino & cujo \\
\hline WPRO\$-F & Elemento relativo CUJO feminino & cuja \\
\hline WPRO\$-F-P & Elemento relativo CUJO feminino plural & cujas, cuja \\
\hline WPRO\$-P & Elemento relativo CUJO masculino plural & cujos, quais \\
\hline WPRO-F & Elemento relativo eminino & quanta \\
\hline WPRO-F-P & Elemento relativo feminino plural & quantas, quais \\
\hline WPRO-P & Elemento relativo plural & quais, quantos, quaes, quantas \\
\hline WQ & Elemento exclamativo/interrogativo & se, como \\
\hline $\mathrm{xx}$ & Palavras desconhecidas & $\mathrm{x}$, tamalavez, $\mathrm{a}$, al, dist \\
\hline
\end{tabular}


B

\section{Avaliação modelo BLSTM-WE-CE no corpus Tycho Brahe.}

$\mathrm{Na}$ Tabela B.1 apresentamos os valores de $F_{1}$, precision e recall médio para cada etiqueta do corpus Tycho Brahe. Os valores médio foram calculados a partir de 10 experimentos realizados com inicialização de sementes aleatórias.

Tabela B.1: Valor médio de $F_{1}$, Precision e Recall por etiqueta no conjunto de treino do corpus Tycho Brahe

\begin{tabular}{|c|c|c|c|}
\hline Classe & $\boldsymbol{F}_{\mathbf{1}} \pm \sigma$ & $\operatorname{Precision} \pm \sigma$ & Recall $\pm \sigma$ \\
\hline( & $1.000 \pm 0.000$ & $1.000 \pm 0.000$ & $1.000 \pm 0.000$ \\
\hline & $1.000 \pm 0.000$ & $1.000 \pm 0.000$ & $1.000 \pm 0.000$ \\
\hline & $1.000 \pm 0.000$ & $1.000 \pm 0.000$ & $1.000 \pm 0.000$ \\
\hline ADJ & $0.912 \pm 0.004$ & $0.922 \pm 0.010$ & $0.905 \pm 0.016$ \\
\hline ADJ-F & $0.937 \pm 0.007$ & $0.939 \pm 0.015$ & $0.937 \pm 0.008$ \\
\hline ADJ-F-P & $0.940 \pm 0.008$ & $0.935 \pm 0.017$ & $0.944 \pm 0.012$ \\
\hline ADJ-G & $0.942 \pm 0.004$ & $0.938 \pm 0.008$ & $0.945 \pm 0.008$ \\
\hline ADJ-G-P & $0.921 \pm 0.006$ & $0.924 \pm 0.012$ & $0.921 \pm 0.017$ \\
\hline ADJ-P & $0.868 \pm 0.004$ & $0.891 \pm 0.021$ & $0.843 \pm 0.020$ \\
\hline ADJ-R & $0.628 \pm 0.148$ & $0.856 \pm 0.043$ & $0.520 \pm 0.170$ \\
\hline ADJ-R-F & $0.982 \pm 0.010$ & $0.982 \pm 0.024$ & $0.982 \pm 0.006$ \\
\hline ADJ-R-F-P & $0.969 \pm 0.003$ & $0.998 \pm 0.006$ & $0.940 \pm 0.000$ \\
\hline ADJ-R-G & $0.934 \pm 0.016$ & $0.913 \pm 0.013$ & $0.959 \pm 0.039$ \\
\hline ADJ-R-G-P & $0.951 \pm 0.003$ & $0.947 \pm 0.008$ & $0.961 \pm 0.014$ \\
\hline ADJ-R-P & $1.000 \pm 0.000$ & $1.000 \pm 0.000$ & $1.000 \pm 0.000$ \\
\hline ADJ-S & $0.910 \pm 0.038$ & $0.902 \pm 0.074$ & $0.925 \pm 0.014$ \\
\hline ADJ-S-F & $0.875 \pm 0.007$ & $0.876 \pm 0.031$ & $0.873 \pm 0.032$ \\
\hline ADJ-S-F-P & $0.754 \pm 0.061$ & $0.665 \pm 0.109$ & $0.886 \pm 0.044$ \\
\hline ADJ-S-P & $0.882 \pm 0.018$ & $0.878 \pm 0.025$ & $0.885 \pm 0.041$ \\
\hline ADV & $0.979 \pm 0.003$ & $0.980 \pm 0.005$ & $0.978 \pm 0.004$ \\
\hline $\mathrm{ADV}+\mathrm{CL}$ & $0.000 \pm 0.000$ & $0.000 \pm 0.000$ & $0.000 \pm 0.000$ \\
\hline ADV-NEG & $0.902 \pm 0.053$ & $0.877 \pm 0.015$ & $0.935 \pm 0.111$ \\
\hline ADV-R & $0.989 \pm 0.003$ & $0.988 \pm 0.004$ & $0.989 \pm 0.003$ \\
\hline ADV-S & $0.000 \pm 0.000$ & $0.000 \pm 0.000$ & $0.000 \pm 0.000$ \\
\hline $\mathrm{C}$ & $0.880 \pm 0.000$ & $0.868 \pm 0.015$ & $0.890 \pm 0.016$ \\
\hline $\mathrm{CL}$ & $0.981 \pm 0.003$ & $0.983 \pm 0.005$ & $0.986 \pm 0.005$ \\
\hline $\mathrm{CL}+\mathrm{CL}$ & $0.958 \pm 0.004$ & $0.967 \pm 0.007$ & $0.958 \pm 0.004$ \\
\hline CONJ & $0.981 \pm 0.003$ & $0.990 \pm 0.000$ & $0.980 \pm 0.000$ \\
\hline CONJ-NEG & $0.936 \pm 0.005$ & $0.885 \pm 0.005$ & $0.992 \pm 0.010$ \\
\hline CONJS & $0.912 \pm 0.006$ & $0.942 \pm 0.012$ & $0.885 \pm 0.017$ \\
\hline $\mathrm{D}$ & $0.990 \pm 0.000$ & $0.994 \pm 0.005$ & $0.990 \pm 0.000$ \\
\hline D+OUTRO & $0.000 \pm 0.000$ & $0.000 \pm 0.000$ & $0.000 \pm 0.000$ \\
\hline D-F & $0.970 \pm 0.000$ & $0.969 \pm 0.006$ & $0.978 \pm 0.004$ \\
\hline D-F-P & $0.989 \pm 0.003$ & $0.977 \pm 0.005$ & $0.995 \pm 0.005$ \\
\hline D-G & $0.048 \pm 0.081$ & $0.084 \pm 0.164$ & $0.087 \pm 0.216$ \\
\hline $\mathrm{D}-\mathrm{G}-\mathrm{P}$ & $0.000 \pm 0.000$ & $0.000 \pm 0.000$ & $0.000 \pm 0.000$ \\
\hline D-P & $0.998 \pm 0.004$ & $0.990 \pm 0.000$ & $0.999 \pm 0.003$ \\
\hline D-UM & $1.000 \pm 0.000$ & $0.990 \pm 0.000$ & $1.000 \pm 0.000$ \\
\hline D-UM-F & $1.000 \pm 0.000$ & $1.000 \pm 0.000$ & $1.000 \pm 0.000$ \\
\hline D-UM-F-P & $0.989 \pm 0.003$ & $0.980 \pm 0.000$ & $0.998 \pm 0.006$ \\
\hline D-UM-P & $1.000 \pm 0.000$ & $1.000 \pm 0.000$ & $1.000 \pm 0.000$ \\
\hline DEM & $0.998 \pm 0.004$ & $0.998 \pm 0.004$ & $0.991 \pm 0.003$ \\
\hline ET & $0.975 \pm 0.005$ & $0.986 \pm 0.014$ & $0.968 \pm 0.012$ \\
\hline ET-D & $0.974 \pm 0.005$ & $0.961 \pm 0.006$ & $0.989 \pm 0.011$ \\
\hline ET-F & $0.900 \pm 0.000$ & $1.000 \pm 0.000$ & $0.820 \pm 0.000$ \\
\hline ET-G & $0.990 \pm 0.011$ & $0.985 \pm 0.016$ & $1.000 \pm 0.000$ \\
\hline ET-I & $0.201 \pm 0.324$ & $0.300 \pm 0.483$ & $0.150 \pm 0.242$ \\
\hline ET-P & $0.990 \pm 0.000$ & $0.989 \pm 0.003$ & $0.990 \pm 0.000$ \\
\hline $\mathrm{ET}-\mathrm{P}+\mathrm{CL}$ & $0.050 \pm 0.158$ & $0.033 \pm 0.104$ & $0.100 \pm 0.316$ \\
\hline ET-PP & $0.944 \pm 0.068$ & $1.000 \pm 0.000$ & $0.899 \pm 0.117$ \\
\hline ET-R & $1.000 \pm 0.000$ & $1.000 \pm 0.000$ & $1.000 \pm 0.000$ \\
\hline ET-RA & $0.942 \pm 0.085$ & $0.899 \pm 0.143$ & $1.000 \pm 0.000$ \\
\hline ET-SD & $0.230 \pm 0.221$ & $0.199 \pm 0.180$ & $0.284 \pm 0.296$ \\
\hline ET-SP & $0.928 \pm 0.019$ & $0.955 \pm 0.051$ & $0.903 \pm 0.045$ \\
\hline ET-SR & $1.000 \pm 0.000$ & $1.000 \pm 0.000$ & $1.000 \pm 0.000$ \\
\hline $\mathrm{FP}$ & $0.925 \pm 0.005$ & $0.935 \pm 0.008$ & $0.912 \pm 0.015$ \\
\hline FW & $0.952 \pm 0.006$ & $0.963 \pm 0.014$ & $0.943 \pm 0.009$ \\
\hline HV & $1.000 \pm 0.000$ & $1.000 \pm 0.000$ & $1.000 \pm 0.000$ \\
\hline $\mathrm{HV}+\mathrm{CL}$ & $0.800 \pm 0.270$ & $0.736 \pm 0.348$ & $1.000 \pm 0.000$ \\
\hline $\mathrm{HV}+\mathrm{SE}$ & $0.634 \pm 0.457$ & $0.650 \pm 0.474$ & $0.650 \pm 0.474$ \\
\hline HV-AN & $0.000 \pm 0.000$ & $0.000 \pm 0.000$ & $0.000 \pm 0.000$ \\
\hline HV-AN-F & $0.000 \pm 0.000$ & $0.000 \pm 0.000$ & $0.000 \pm 0.000$ \\
\hline
\end{tabular}




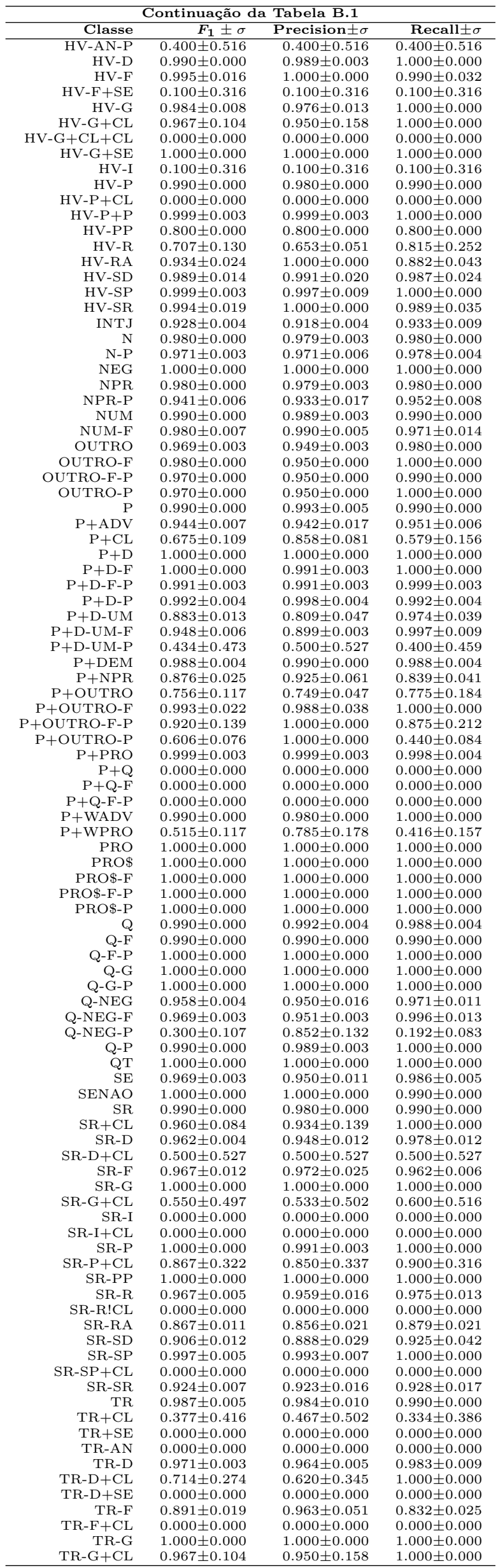




\begin{tabular}{|c|c|c|c|}
\hline \multicolumn{4}{|c|}{ Continuação da Tabela B.1 } \\
\hline Classe & $\boldsymbol{F}_{\mathbf{1}} \pm \sigma$ & $\operatorname{Precision} \pm \sigma$ & Recall $\pm \sigma$ \\
\hline TR-G+SE & $0.900 \pm 0.316$ & $0.900 \pm 0.316$ & $0.900 \pm 0.316$ \\
\hline TR-I & $1.000 \pm 0.000$ & $1.000 \pm 0.000$ & $1.000 \pm 0.000$ \\
\hline $\mathrm{TR}-\mathrm{I}+\mathrm{CL}$ & $0.000 \pm 0.000$ & $0.000 \pm 0.000$ & $0.000 \pm 0.000$ \\
\hline TR-P & $0.990 \pm 0.000$ & $0.980 \pm 0.000$ & $0.997 \pm 0.005$ \\
\hline $\mathrm{TR}-\mathrm{P}+\mathrm{CL}$ & $0.506 \pm 0.160$ & $0.547 \pm 0.081$ & $0.515 \pm 0.253$ \\
\hline $\mathrm{TR}-\mathrm{P}+\mathrm{SE}$ & $0.300 \pm 0.483$ & $0.300 \pm 0.483$ & $0.300 \pm 0.483$ \\
\hline TR-PP & $0.700 \pm 0.000$ & $0.540 \pm 0.000$ & $1.000 \pm 0.000$ \\
\hline TR-R & $0.990 \pm 0.000$ & $0.980 \pm 0.000$ & $1.000 \pm 0.000$ \\
\hline TR-R!CL & $0.000 \pm 0.000$ & $0.000 \pm 0.000$ & $0.000 \pm 0.000$ \\
\hline TR-RA & $0.926 \pm 0.013$ & $0.942 \pm 0.004$ & $0.902 \pm 0.025$ \\
\hline TR-SD & $0.980 \pm 0.000$ & $1.000 \pm 0.000$ & $0.970 \pm 0.000$ \\
\hline TR-SP & $0.976 \pm 0.005$ & $0.970 \pm 0.011$ & $0.981 \pm 0.003$ \\
\hline TR-SP+CL & $0.602 \pm 0.088$ & $0.800 \pm 0.258$ & $0.500 \pm 0.000$ \\
\hline TR-SR & $0.871 \pm 0.023$ & $0.894 \pm 0.052$ & $0.850 \pm 0.046$ \\
\hline VB & $0.981 \pm 0.003$ & $0.982 \pm 0.004$ & $0.987 \pm 0.005$ \\
\hline $\mathrm{VB}+\mathrm{CL}$ & $0.914 \pm 0.022$ & $0.873 \pm 0.048$ & $0.958 \pm 0.010$ \\
\hline $\mathrm{VB}+\mathrm{CL}+\mathrm{CL}$ & $0.020 \pm 0.063$ & $0.033 \pm 0.104$ & $0.014 \pm 0.044$ \\
\hline $\mathrm{VB}+\mathrm{SE}$ & $0.967 \pm 0.012$ & $0.961 \pm 0.020$ & $0.974 \pm 0.013$ \\
\hline $\mathrm{VB}+\mathrm{SE}+\mathrm{CL}$ & $0.000 \pm 0.000$ & $0.000 \pm 0.000$ & $0.000 \pm 0.000$ \\
\hline VB-AN & $0.922 \pm 0.004$ & $0.917 \pm 0.013$ & $0.932 \pm 0.011$ \\
\hline VB-AN-F & $0.932 \pm 0.006$ & $0.934 \pm 0.010$ & $0.931 \pm 0.007$ \\
\hline VB-AN-F-P & $0.942 \pm 0.010$ & $0.937 \pm 0.022$ & $0.946 \pm 0.008$ \\
\hline VB-AN-P & $0.915 \pm 0.005$ & $0.928 \pm 0.011$ & $0.904 \pm 0.016$ \\
\hline VB-D & $0.946 \pm 0.005$ & $0.938 \pm 0.014$ & $0.958 \pm 0.006$ \\
\hline $\mathrm{VB}-\mathrm{D}+\mathrm{CL}$ & $0.797 \pm 0.016$ & $0.848 \pm 0.070$ & $0.759 \pm 0.066$ \\
\hline VB-D+SE & $0.863 \pm 0.016$ & $0.861 \pm 0.069$ & $0.875 \pm 0.054$ \\
\hline $\mathrm{VB}-\mathrm{D}+\mathrm{SE}+\mathrm{CL}$ & $0.017 \pm 0.054$ & $0.014 \pm 0.044$ & $0.020 \pm 0.063$ \\
\hline VB-F & $0.900 \pm 0.014$ & $0.890 \pm 0.037$ & $0.917 \pm 0.035$ \\
\hline $\mathrm{VB}-\mathrm{F}+\mathrm{CL}$ & $0.000 \pm 0.000$ & $0.000 \pm 0.000$ & $0.000 \pm 0.000$ \\
\hline $\mathrm{VB}-\mathrm{F}+\mathrm{CL}+\mathrm{CL}$ & $0.000 \pm 0.000$ & $0.000 \pm 0.000$ & $0.000 \pm 0.000$ \\
\hline $\mathrm{VB}-\mathrm{F}+\mathrm{SE}$ & $0.656 \pm 0.101$ & $0.905 \pm 0.113$ & $0.530 \pm 0.125$ \\
\hline VB-G & $0.985 \pm 0.005$ & $0.985 \pm 0.005$ & $0.984 \pm 0.005$ \\
\hline $\mathrm{VB}-\mathrm{G}+\mathrm{CL}$ & $0.811 \pm 0.052$ & $0.780 \pm 0.075$ & $0.855 \pm 0.075$ \\
\hline VB-G+SE & $0.914 \pm 0.029$ & $0.921 \pm 0.050$ & $0.909 \pm 0.052$ \\
\hline $\mathrm{VB}-\mathrm{G}+\mathrm{SE}+\mathrm{CL}$ & $0.058 \pm 0.078$ & $0.048 \pm 0.070$ & $0.080 \pm 0.103$ \\
\hline VB-I & $0.701 \pm 0.012$ & $0.821 \pm 0.069$ & $0.617 \pm 0.043$ \\
\hline $\mathrm{VB}-\mathrm{I}+\mathrm{CL}$ & $0.513 \pm 0.040$ & $0.700 \pm 0.151$ & $0.428 \pm 0.098$ \\
\hline VB-P & $0.960 \pm 0.000$ & $0.964 \pm 0.007$ & $0.958 \pm 0.004$ \\
\hline VB-P+CL & $0.744 \pm 0.037$ & $0.740 \pm 0.114$ & $0.763 \pm 0.052$ \\
\hline $\mathrm{VB}-\mathrm{P}+\mathrm{CL}+\mathrm{CL}$ & $0.000 \pm 0.000$ & $0.000 \pm 0.000$ & $0.000 \pm 0.000$ \\
\hline VB-P $+\mathrm{P}$ & $0.900 \pm 0.316$ & $0.900 \pm 0.316$ & $0.900 \pm 0.316$ \\
\hline VB-P+SE & $0.897 \pm 0.014$ & $0.895 \pm 0.035$ & $0.900 \pm 0.016$ \\
\hline $\mathrm{VB}-\mathrm{P}+\mathrm{SE}+\mathrm{CL}$ & $0.252 \pm 0.077$ & $0.579 \pm 0.310$ & $0.185 \pm 0.092$ \\
\hline VB-PP & $0.924 \pm 0.005$ & $0.936 \pm 0.017$ & $0.913 \pm 0.016$ \\
\hline VB-R & $0.909 \pm 0.011$ & $0.910 \pm 0.036$ & $0.913 \pm 0.026$ \\
\hline VB-R!CL & $0.249 \pm 0.110$ & $0.375 \pm 0.207$ & $0.225 \pm 0.115$ \\
\hline VB-R!SE & $0.141 \pm 0.177$ & $0.304 \pm 0.375$ & $0.100 \pm 0.133$ \\
\hline VB-RA & $0.816 \pm 0.017$ & $0.897 \pm 0.047$ & $0.751 \pm 0.022$ \\
\hline VB-RA+CL & $0.000 \pm 0.000$ & $0.000 \pm 0.000$ & $0.000 \pm 0.000$ \\
\hline VB-RA+SE & $0.000 \pm 0.000$ & $0.000 \pm 0.000$ & $0.000 \pm 0.000$ \\
\hline VB-SD & $0.962 \pm 0.006$ & $0.959 \pm 0.017$ & $0.962 \pm 0.010$ \\
\hline VB-SD+CL & $0.000 \pm 0.000$ & $0.000 \pm 0.000$ & $0.000 \pm 0.000$ \\
\hline VB-SP & $0.918 \pm 0.010$ & $0.938 \pm 0.033$ & $0.901 \pm 0.017$ \\
\hline $\mathrm{VB}-\mathrm{SP}+\mathrm{CL}$ & $0.683 \pm 0.048$ & $0.684 \pm 0.131$ & $0.705 \pm 0.064$ \\
\hline VB-SP+SE & $0.794 \pm 0.069$ & $0.819 \pm 0.165$ & $0.800 \pm 0.077$ \\
\hline $\mathrm{VB}-\mathrm{SP}+\mathrm{SE}+\mathrm{CL}$ & $0.000 \pm 0.000$ & $0.000 \pm 0.000$ & $0.000 \pm 0.000$ \\
\hline VB-SR & $0.755 \pm 0.021$ & $0.832 \pm 0.049$ & $0.695 \pm 0.058$ \\
\hline WADV & $0.870 \pm 0.007$ & $0.916 \pm 0.020$ & $0.830 \pm 0.023$ \\
\hline WD & $0.804 \pm 0.013$ & $0.819 \pm 0.037$ & $0.793 \pm 0.034$ \\
\hline WD-F & $0.791 \pm 0.083$ & $0.854 \pm 0.175$ & $0.760 \pm 0.084$ \\
\hline WD-F-P & $0.621 \pm 0.248$ & $0.672 \pm 0.094$ & $0.636 \pm 0.334$ \\
\hline WD-P & $0.593 \pm 0.033$ & $0.666 \pm 0.151$ & $0.557 \pm 0.049$ \\
\hline WPRO & $0.920 \pm 0.000$ & $0.917 \pm 0.007$ & $0.929 \pm 0.012$ \\
\hline WPRO\$ & $1.000 \pm 0.000$ & $1.000 \pm 0.000$ & $1.000 \pm 0.000$ \\
\hline WPRO\$-F & $1.000 \pm 0.000$ & $1.000 \pm 0.000$ & $1.000 \pm 0.000$ \\
\hline WPRO\$-F-P & $1.000 \pm 0.000$ & $1.000 \pm 0.000$ & $1.000 \pm 0.000$ \\
\hline WPRO\$-P & $1.000 \pm 0.000$ & $1.000 \pm 0.000$ & $1.000 \pm 0.000$ \\
\hline WPRO-F & $0.431 \pm 0.249$ & $0.299 \pm 0.185$ & $0.800 \pm 0.422$ \\
\hline WPRO-F-P & $0.576 \pm 0.153$ & $0.496 \pm 0.099$ & $0.701 \pm 0.247$ \\
\hline WPRO-P & $0.883 \pm 0.018$ & $0.851 \pm 0.011$ & $0.920 \pm 0.042$ \\
\hline WQ & $0.641 \pm 0.030$ & $0.748 \pm 0.073$ & $0.566 \pm 0.049$ \\
\hline XX & $0.000 \pm 0.000$ & $0.000 \pm 0.000$ & $0.000 \pm 0.000$ \\
\hline
\end{tabular}

